\title{
MEDIA LITERACY EDUCATION IN HIGHER EDUCATION POLICY: PEDAGOGICAL CHOICES AND LEARNING OUTCOMES
}

\author{
A Dissertation \\ presented to \\ the Faculty of the Graduate School \\ at the University of Missouri-Columbia \\ In Partial Fulfillment \\ of the Requirements for the Degree \\ Doctor of Education \\ by \\ BRAD MITCHELL \\ Dr. Sarah Diem, Dissertation Supervisor \\ DECEMBER 2020
}


The undersigned, appointed by the dean of the Graduate School, have examined the dissertation entitled

\section{MEDIA LITERACY EDUCATION IN HIGHER EDUCATION POLICY: PEDAGOGICAL CHOICES AND LEARNING OUTCOMES}

presented by Brad Mitchell,

a candidate for the degree of doctor of education,

and hereby certify that, in their opinion, it is worthy of acceptance.

Professor Sarah Diem

Professor Jennifer Fellabaum-Toston

Professor Lissa Behm-Morawitz

Professor Danna Wren 


\section{ACKNOWLEDGEMENTS}

I would like to thank the following people for helping me undertake, and most importantly finish, this research: my advisor and committee chair, Dr. Sarah Diem, for her patience and expertise helping me throughout the dissertation writing process, Dr. Blake Naughton for helping me start this process and identifying a research topic, and Drs. Lissa Behm-Morawitz, Jennifer Fellabaum-Toston, and Danna Wren for serving on my dissertation committee. I would also like to thank Dr. Cynthia Macgregor for taking a chance on me and helping shepherd me through the Statewide Cooperative EdD Program the first two years I was in the program and based at Missouri State University.

Thank you to my wife Erica for her unwavering patience and support - I could not have done this without you. Finally, thanks to Fluffy for helping entertain Jameson when I needed to write, and thanks to Jameson for simply being you. 
TABLE OF CONTENTS

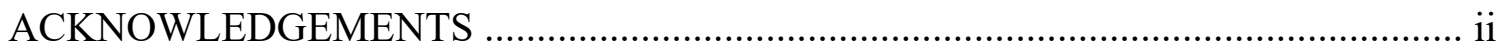

LIST OF FIGURES ....................................................................................... iv

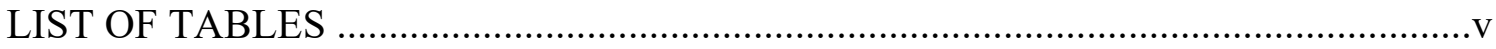

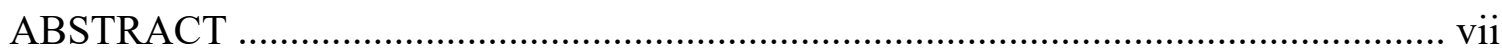

Chapter

1. INTRODUCTION TO THE DISSERTATION-IN-PRACTICE .....................1

2. PRACTITIONER SETTING FOR THE STUDY …....................................

3. SCHOLARLY REVIEW FOR THE STUDY .............................................65

4. CONTRIBUTION TO PRACTICE ..................................................... 186

5. CONTRIBUTION TO SCHOLARSHIP .................................................195

6. SCHOLARLY PRACTITIONER REFLECTION.....................................227

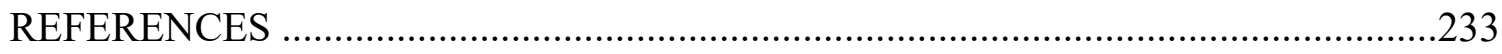

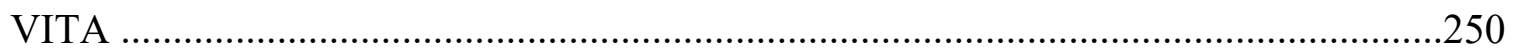




\section{LIST OF FIGURES}

Figure

Page

1. Participatory Ecological Framework of Media Literacy ....................12

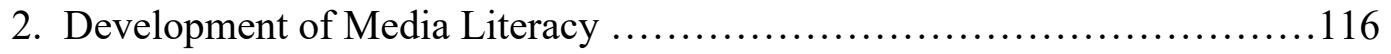

3. Key Questions to Ask When Analyzing Media Messages .................121

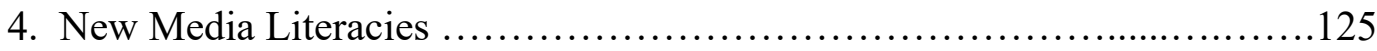

5. Critical Media Production Model ..................................... 132

6. Critical Media Literacy Questions and Concepts ..........................136

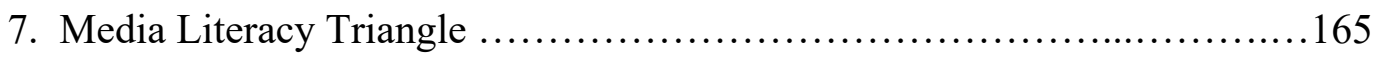

8. Participatory Ecological Framework of Media Literacy ....................172

9. Example Scenario \#1 ................................................ 177

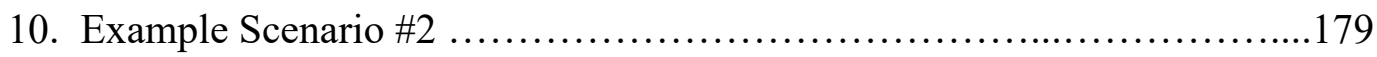

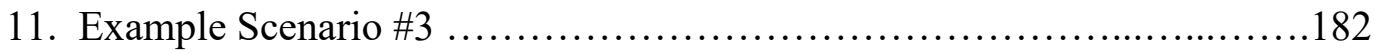

12. Participatory Ecological Framework of Media Literacy ..................189

13. Participatory Ecological Framework of Media Literacy ...................207 


\section{LIST OF TABLES}

Table Page

1. Criteria for Participant Data Collection Sites ...............................................16

2. Faculty Participant Demographics .................................... 21

3. Findings on the Inclusion of Participatory Methods of Learning.............191

4. Criteria for Participant Data Collection Sites ...........................................2211

5. Faculty Participant Demographics ..................................212

6. Findings on the Inclusion of Participatory Methods of Learning .............216 


\begin{abstract}
This study proposes a participatory pedagogical framework founded on critical thinking as a method for analysis, reflection, and creative production of media through an understanding of the ways in which media literacy is being taught in higher education institutions in the United States. A qualitative design was utilized that included techniques of content and ethnographic analysis to focus on shared patterns across a sample of higher education courses in the U.S. containing elements of media literacy instruction. This study has found inconsistencies in the pedagogical approaches being utilized, a misunderstanding of the critical thinking and inquiry-based learning methods required for a critical field such as media literacy, and the lack of administrative oversight or policy support as key factors contributing to the discrepancies in media literacy's instruction and learning outcomes. Implications include recommendations for the field to consolidate theoretical frameworks, modifications to teaching, and policy and administrative advocacy efforts.
\end{abstract}


Running head: MEDIA LITERACY IN HIGHER EDUCATION POLICY

Chapter One: Introduction to the Dissertation-in-Practice 


\section{Background}

We live today with an abundance of technological tools available at our fingertips at any given moment. The proliferation of high-speed Internet access, social media, mobile devices, and other digital technologies allow for exposure to media messages at ever increasing rates. With many Americans currently living in a reality of constant connectedness and information overload, the allocation of a scarce resource such as individual attention spans should be focused on relevant messages that produce value in our everyday experience.

Literacy is having the knowledge and skills to read, understand, and produce texts that allow one to participate in society (Kellner \& Share, 2005). The traditional view of literacy as the ability to read and write has now expanded to include digital and media literacies. The definition of text now includes more than the written word, rather any message form whether it be visual, aural, verbal, interactive, or a combination thereof (National Association for Media Literacy Education, 2014; Thoman \& Jolls, 2003). Media texts, specifically, are those images, sounds, and programs transmitted by different communication technologies such as web sites, mobile applications, film, radio, television, and print (Buckingham, 2003). Literacy in the context of media encompasses the effective use of communication forms and representations (Kellner \& Share, 2005). As we continue to interact with the media on a constant basis, citizens are realizing that our relationship with media is not transitory, instead this association is perpetual and requires a continued examination over time.

Media literacy requires higher-order creative and critical thinking skills, meaning that people can access, analyze, evaluate and create media in order to participate fully in 
our digitally augmented society (Aufderheide, 1993; RobbGrieco \& Hobbs, 2013). Media literacy stresses critical thinking and creativity not only for students to be critical consumers of media, but also to themselves become producers and communicators (Buckingham, 2003). A practical understanding of this form of literacy is a basis for effective knowledge production and active civic engagement in a democratic society.

The education system in the United States has given little attention to media literacy in the past (Heins \& Cho, 2003); however, the subject has been making inroads in recent years as educators realize this pedagogy is crucial to 21 st century learning (Jolls et al., 2016). This new form of literacy is especially affecting how higher education institutions address digital literacies in their curriculum objectives and teacher development programs. A lack of consistency in pedagogy and assessment for media literacy courses in post-secondary education is hindering many universities from formulating adequate policies and programs that address this challenge (Literat, 2014; Mihailidis, 2008). While the basic definition of media literacy has been generally accepted for decades, the interpretation of that definition varies broadly among different academic disciplines (Mihailidis, 2008). Compounding this issue is the notion that media literacy encompasses skills that differ for educators and learners, as teaching with media is inherently different from learning through it.

Media literacy pedagogy is unique because it can be seen as a subject unto itself, but it can also be about any subject, or can be used as a way to navigate all subjects; it offers a common base of understanding through which any content or philosophy can be examined (Jolls et al., 2016). This form of literacy promotes social justice, an understanding of current technology and communication skills, and comprehensive global 
competencies. An understanding of media literacy leads to questions instead of answers, critical analysis in place of regulation, and empowers learners rather than fitting them into preselected roles. It is a critical pedagogy that leads to transformative learning and attached to its definition are challenging ramifications that must be addressed. This criti-

cal pedagogy is at odds with our current education system based on neoliberal ideals with a focus on standardization and privatization (RobbGrieco \& Hobbs, 2013).

Unlike many other developed nations, the U.S. is currently lacking in national policy support for media literacy education. For instance, in 2007 the European Commission mandated that all member states develop an assessment strategy for measuring the media literacy competence of its citizens (European Union, 2007), leading to the accumulation of substantial datasets and research coming out of the E.U. While there are many areas of innovation occurring in the U.S., they tend to be local in scope (RobbGrieco \& Hobbs, 2013). The inclusion of media literacy education in university policy could ensure that consistent, appropriate pedagogy and learning outcomes are established, as well as address any institutional challenges possibly being faced by faculty. It is of absolute urgency and importance that educational policies be drafted that address media literacy competency for students in higher education.

\section{Statement of the Problem}

Nations such as the Nordic countries, Canada, the United Kingdom, and all European Union member states have developed domestic policies that guide the teaching of media literacy in elementary, secondary, and post-secondary educational settings (Andersen et al., 2013; European Union, 2007; Lundgren, et all, 2013). In some of these coun- 
tries, media literacy is assessed on a national level, while in others such as the U.S., evaluation is taking place most often at state and local levels; these inconsistencies have led to variances of media literacy's inclusion in the curriculum and the ways in which learning outcomes are measured (Schilder et al., 2016). While other developed countries explicitly recognize the teaching of media literacy as a pedagogical necessity, the concept of media literacy in U.S. educational policy is not as straightforward.

First, media literacy is still fairly unknown in the U.S., and has little support or awareness from administrators or institutional policy (Kellner \& Share, 2005). Second, in the U.S., the terms digital literacy or technological literacy are commonplace. These terms are marginally related to media literacy, but the concept of digital literacy suggests a more practical approach. Digital literacy can be seen as containing some aspects of media literacy; however, media literacy is understood to be a more complex competency with an emphasis on critical thinking and creativity (National Association for Media Literacy Education, 2014). U.S. educational policies concentrating on media literacy are not as comprehensive as those policies found in the European Union and other developed nations.

This lack of policy support has contributed to the discrepancies in the instruction and learning outcomes for media literacy in higher education, as well as inconsistencies in the scope of how and where it is taught. Mihailidis (2008) states that the, "inconsistencies in definition, use and adoption have ... hindered media literacy's ability to produce tangible and coherent learning outcomes for higher education" (p 1). According to the literature, there is little agreement in the ways that media literacy competencies should be 
assessed, with a need for the creation of standardized measurements (Christ, 2004; Livingstone \& Thumim, 2003). Additionally, media literacy education research has most commonly focused on PK-12 education, with a lack of study for higher education settings (Koltay, 2011; Schmidt, 2013).

The focus of media literacy education should be the use of media content to meet learning outcomes; however, there is a gap in the literature about what learning has actually taken place (Mihailidis, 2008). Assessment tools for media literacy education are also scarce, and educators have emphasized the need for a national standard of media literacy assessment (Literat, 2014; Schilder et al., 2016). Finally, there is a lack of consistency in pedagogy, with competition taking place among the conceptual models through which media literacy is taught. These pedagogical conflicts include the ecological model versus the big media model (Andersen et al., 2013), and the protectionist lens versus the empowerment lens (Mihailidis, 2009).

\section{Purpose of the Study}

The purpose of this study is to inform university policy development in regard to media literacy education by determining the pedagogical approaches and learning outcomes of media literacy instruction in higher education. This was accomplished through (a) a comprehensive review of the literature, (b) the creation of a theoretical framework for media literacy instruction in higher education based on a synthesis of the pedagogical approaches and learning outcomes found in the literature, (c) a qualitative study of 24 un-

dergraduate courses that include elements of media literacy instruction, (d) a comparison of the findings from the qualitative study against the newly created theoretical framework, and (e) recommendations for future university policy development regarding media 
literacy education. This evidence-informed approach will be a reliable scholarly foundation for practical policy recommendations.

\section{Research Questions}

In the absence of national policy regarding media literacy instruction in higher education settings, the following research questions guided this study:

1. How is media literacy being taught in higher education institutions in the U.S.?

a. In what ways is the pedagogical approach of analysis included in this instruction?

b. In what ways is the pedagogical approach of production included in this instruction?

c. In what ways is the pedagogical approach of reflection included in this instruction?

d. In what ways are all three of these components integrated together?

2. What are the intended learning outcomes of media literacy instruction in higher education institutions in the U.S.?

3. What environmental factors influence the teaching of media literacy in higher education institutions in the U.S.?

a. What variations are found between public and private institutions of higher education?

b. How significant is policy to the environments in which media literacy is taught?

\section{Conceptual and Theoretical Frameworks}


Our society has reached a saturation point in the amount of information we are subjected to on a daily basis, with no single piece of information having any distinctive significance (Potter, 2004). This overabundance of communication creates difficulty in detecting opinion from fact, inaccuracy from validity, or devalued media messages from invaluable ones (Potter, 2004; Thoman \& Jolls, 2003). Media literacy is the viewpoint from which we access the media, critically evaluate its content, and create meanings from the images, words, and sounds in the messages we encounter (Aufderheide, 1993; European Union, 2007). Media literacy education offers a framework for understanding and negotiating meanings through active inquiry, self-expression and critical thinking (Morrell et al., 2013; National Association for Media Literacy Education, 2007; Potter, 2004; Thoman \& Jolls, 2003). This type of education develops engaged, knowledgeable citizens who are acculturated to living in our media-saturated society through lifelong use of their skills and experiences to construct their own meanings from media messages (Hobbs, 2010; National Association for Media Literacy Education, 2007).

These media literacy skills must be established in formal educational settings to ensure active membership in our new participatory culture founded on new technologies and innovative communication platforms (Hobbs, 2010; Jenkins et al., 2009). This participatory culture is defined by Jenkins et al. (2009) as one with, "relatively low barriers to artistic expression and civic engagement, strong support for creating and sharing creations, and some type of informal mentorship whereby experienced participants pass along knowledge to novices" (p. xi). 
The facilitation of effective media literacy education is based on constructivist pedagogy (RobbGrieco \& Hobbs, 2013). This approach allows learners to actively embrace critical thinking and creativity through the applied reflection of analysis, discussion, and engagement with the media (Hobbs, 2010; Literat, 2014). This practical pedagogical focus will help future generations connect their media literacy competencies to emerging ethical situations that will inevitably appear as they navigate citizenship in participatory digital communities (Hobbs, 2010; Jenkins et al., 2009; RobbGrieco \& Hobbs, 2013).

The conceptual frameworks through which media literacy education takes place have evolved over time, and multiple frameworks are currently being utilized in the classroom. Aufderheide's (1993) definition of media literacy being the ability to access, analyze, evaluate, and create messages in a variety of forms is the foundation for most media literacy instructional frameworks. While this definition is concise and contains the essential components of media literacy, it is limited to the extent that it is not sufficient in our current climate of diversification and convergence of media (Livingstone, 2002; 2004).

Buckingham (2003) expanded on this basic definition by adopting a student-centered approach that is positioned around past experiences and pre-existing knowledge of the media. This framework de-emphasizes the protectionist stance of Aufderheide's (1993) original definition, and instead encourages students to make their own informed decisions regarding media interactions (Buckingham, 2003). Potter (2004) continued Buckingham's (2003) transition from a protectionist lens to an empowerment lens with the cognitive model of media literacy. Potter (2004) defines media literacy as having the capacity to, "empower individuals to shift control from the media to themselves" (p. 68) 
through the understanding of one's knowledge structures, personal locus, competencies, and flow of information-processing.

An inquiry-based approach to teaching media literacy is also a well-established method, which uses relevant questions to evaluate and analyze media messages. Specifically, this pedagogical approach is undertaken by teachers, working collaboratively with their students, to link students' personal encounters with the media in order to enhance their overall learning experience (Silverblatt, 2014). This framework is focused on analysis and reflection of media messages through inquiry and is less concerned with media production. Contrasting this approach is the new media literacies framework, which is founded upon the recent convergence of media platforms as defined by media scholar Henry Jenkins. Jenkins (2006) defines media convergence as the collaborative partnership among differing media industries that allows content to appear across multiple platforms. The new media literacies framework addresses issues of understanding how students interact with interactive digital media, while confirming the assumption that higher levels of media literacy translate to increased levels of civic engagement and media participation (Jenkins et al., 2009). While this framework builds on the collaborative approach present in inquiry-based pedagogy, it is lacking in the thoroughness of understanding the cognitive abilities required of a media literate person found in other theoretical frameworks.

Guided by Friere's (2010) work on emancipation via pedagogy and Brookfield et al.'s (2012) research on critical thinking as a reflective thought process, the media production approach is based on the idea that critical pedagogy fosters creativity and critical media production (Morrell et al., 2013). In practice, Morrell et al.'s (2013) model for 
critical media pedagogy positions learning as active, authentic, participatory, and empowering. While this critical approach (Morrell et al., 2013) stresses media production, it corresponds to the literature emphasizing media literacy education's ability to foster participatory democratic engagement through critical access, analysis, communication, and creation of media messages (Buckingham, 2003; European Union, 2007; Hobbs, 2010; Jenkins et al., 2009; Mihailidis, 2008).

Another framework that is highly influenced by Freire's (2010) critical pedagogy framework is that of critical media literacy as a transformative pedagogy (Funk et al., 2016). This framework displays an important method for strengthening civic engagement among students by reaffirming the notion of democracy through empowerment (Funk et al., 2016). Unlike Morrell et al.'s (2013) media production approach, however, the critical media literacy framework emphasizes the analysis of media messages over media production. These two critical frameworks, together, tie directly to the idea of what it means to be an active citizen in today's participatory culture.

The frameworks listed above further explored in the upcoming scholarly review section. The scholarly review created a foundation for media literacy pedagogy based on these oft-cited frameworks, with an aim of developing a comprehensive pedagogical model for higher education at the conclusion of the scholarly review. This newly constructed theoretical framework is called the participatory ecological framework of media literacy (see Figure 1) and used to compare the data findings in order to answers R1 and R2.

The participatory ecological framework of media literacy applies a philosophical approach to pedagogy based on Freire's (2010) guiding principles of critical dialogue, 
critical reflection, and action constituting a transformative pedagogy. In this framework, these principles have been reconstructed into the three media literacy pedagogical approaches of analysis, reflection, and production.

\section{Figure 1}

\section{Participatory Ecological Framework of Media Literacy}

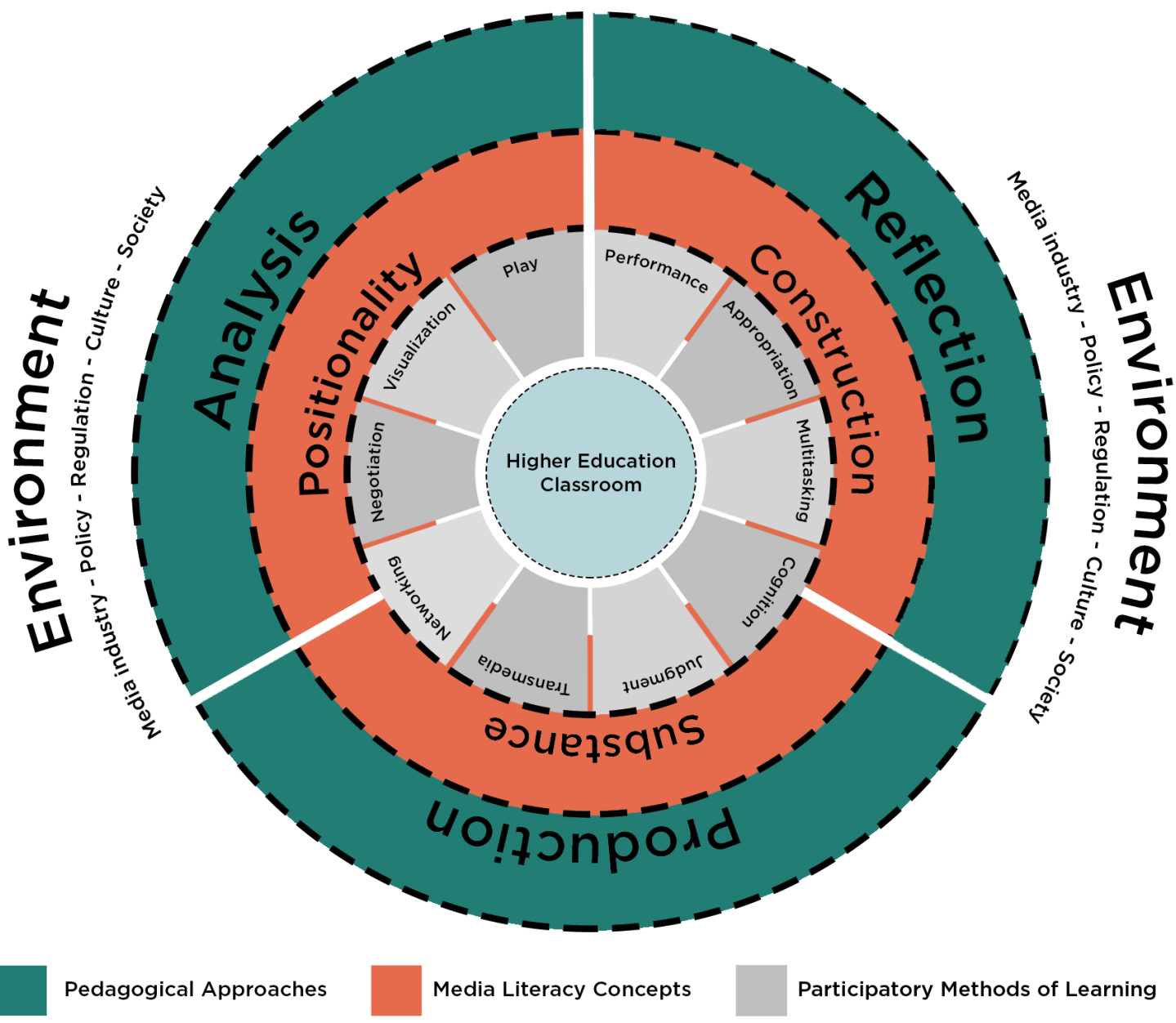

Note. This figure shows the components of the participatory ecological framework, including the pedagogical approaches, media literacy concepts, and participatory methods of learning.

Three requirements are necessary for such a pedagogical framework to be successful in various education settings; those being the possibility of teaching media literacy in multiple academic disciplines, in differing environments, and by faculty with varying levels of 
expertise in traditional media studies. By basing the participatory ecological framework in cultural studies and critical thinking, the subject can be taught across multiple disciplines by addressing socio-economic issues such as gender, race, class, politics, and power through the lens of the appropriate subject area. The participatory ecological framework of media literacy is comprehensively explored at the conclusion of the scholarly review for this study.

\section{Design of the Study}

The research questions in this study were addressed through a qualitative design utilizing the techniques of content analysis and ethnographic analysis. Content analysis is an inductive research approach that determines the presence of particular themes or words within a set of texts (Merriam, 2009). Ethnographic analysis was in the form of qualitative interviews intended to discover a deeper understanding of how institutional policies and culture possibly influenced the teaching of media literacy in the participant settings (Merriam, 2009). Overall, the study focuses on shared patterns across the sample of higher education courses containing elements of media literacy education.

The first set of data analyzed in this study was written documents consisting of course syllabi, program overview statements, general education overview statements, and policy statements from the sample of post-secondary institutions. The second set of data consisted of qualitative interviews with a subset of faculty from the participant settings in order to gain a deeper understanding concerning policies and institutional support for media literacy in their settings. Data was collected from both private and public universities to better understand the possible policy implications in both settings. Documentary data 
was collected from 24 higher education courses that focus on, or include elements of, media literacy instruction, from 24 different institutions. The content analysis of this documentary data was conducted with an underlying goal of discovery and verification, the sampling was purposeful, the types of data were narrative, and the data analysis was textual (Altheide, 1987). The interview data was collected from a subset of 6 faculty members from the participant settings in the form of follow-up interviews at the conclusion of the collection and analysis of the documentary data.

Transferability of the findings to future policy was achieved through the contribution to practice and scholarship sections intended for current policy stakeholders (EPPICentre, 2007; Pigott et al., 2009). The policy and practice recommendations proposed from this study were explicitly connected to the scholarly review, the newly constructed theoretical framework, and the primary data findings in order to ensure the evidencebased approach is clearly understood by policymakers (EPPI-Centre, 2007). The sizable number of data collection sites was intended to better the generalizability of the results (Creswell, 2007). Allowing for maximum variability within the sample also enhances the transferability of this qualitative study (Merriam, 2009).

The study was particularistic in design, since its focus is on a specific category of a higher education academic subject area (Gay et al., 2009; Merriam, 2009). The study was also descriptive through its utilization of as many variables as possible from the documentary data and the follow-up interviews (Merriam, 2009). This focused study design is well suited to investigating a practical problem such as approaches to media literacy education and the implications for its future policy development (Sykes et al., 2009; Merriam, 2009). 


\section{Setting and Participants}

The setting of this study consists of 24 higher education courses that focus on, or include elements of, media literacy instruction, from 24 different higher education institutions. Specifically, the setting includes 12 four-year private universities and 12 four-year public universities in the U.S.; this substantial sample size assists with ensuring as much variability as possible among the institutional settings. Multiple characteristics, while not included in the criteria for participant data collection sites, were also considered with an aim of diversification among the institutions. These aspects included the geographic location of the institution, whether it is a research or teaching university, and how other internal and external sociopolitical factors might contribute to decisions made during policy design and implementation.

Data was collected from these 24 sites based on the following categories: (a) mass media and communication studies theoretical prerequisite courses, (b) general education courses, (c) teacher education courses, and (d) courses in various subject areas that integrate elements of media literacy education into the course content (see Table 1). Data was collected from three private four-year universities and three public four-year universities within each category, thus equaling 24 total sites for data collection. 


\section{Table 1}

Criteria for Participant Data Collection Sites

\begin{tabular}{lllll}
\hline & $\begin{array}{l}\text { Mass media } \\
\text { and communi- } \\
\text { cation theoreti- } \\
\text { cal prerequisite } \\
\text { courses }\end{array}$ & $\begin{array}{l}\text { General } \\
\text { education } \\
\text { courses }\end{array}$ & $\begin{array}{l}\text { Teacher } \\
\text { education } \\
\text { courses }\end{array}$ & $\begin{array}{l}\text { Integrated } \\
\text { courses within } \\
\text { various other } \\
\text { subject areas }\end{array}$ \\
\hline $\begin{array}{l}\text { Public 4-year } \\
\begin{array}{l}\text { institutions } \\
\text { Private 4-year } \\
\text { institutions }\end{array}\end{array}$ & 3 courses & 3 courses & 3 courses & 3 courses \\
\hline
\end{tabular}

From these 24 participant sites, a smaller subset was selected for follow-up interviews at the conclusion of the collection and analysis of the documentary data. These interviews were intended to better understand how issues of institutional support, policy, and environmental factors influence the teaching of media literacy. The total number of qualitative interviews was 6; four interviews were conducted with faculty from private institutions, with two additional interviews taking place with faculty from public university settings. The sampling of participant sites in this study can be seen as both convenient, due to the sample size not including the entire population of possible higher education courses in the U.S. containing elements of media literacy instruction (Krippendorff, 2004), as well as purposeful, since the sampling of participant sites are based on specific criteria (Merriam, 2009).

One criterion of all 24 settings was that the courses are taught at the undergraduate level; courses taught at the masters or doctoral level were not considered. There were no criteria in place requiring a setting to have either a school or academic program focusing on subject areas closely aligned with media literacy such as mass media studies, communication, or journalism. Since data was collected from various academic subject areas, 
and because media literacy is currently being taught in multiple academic disciplines (Silverblatt, 2014), this requirement was not in place for data collection to occur. Furthermore, the term media literacy was not required to be in the course title of any particular sample. As will be explained in further detail in the scholarly review, the language used to define media literacy in higher education is ambiguous and inconsistent (Buckingham, 2009; Mihailidis, 2008), with terms such as digital literacy, digital media literacy, or new media literacy often seen as being interchangeable.

It was not required that any of the data collection sites include all three pedagogical approaches found in the participatory ecological framework of media literacy; those being analysis, reflection, and production. It was a requirement, however, for each data collection site to include at least one of these pedagogical approaches in their curriculum in order to reliably compare findings against other sites as well as with the theoretical framework introduced in this study. It was expected that analysis would reveal a multitude of combinations of the pedagogical approaches, media literacy concepts, and participatory methods of learning that are found in the participatory ecological framework, as well as other components that are not included in the framework.

There was no expectation that the courses were traditional seated courses, hybrid, or online courses; data was collected regardless of the instructional design of the course. Additionally, no restrictions were placed on course length; for example, traditional 16week semester courses, shorter intensive summer courses, or any other course length was considered valid with the understanding that the course is offered to students for academic credit. The use of multiple, varying sites in different academic settings and disciplines contributed to the contextual variations, or lack thereof, that emerge in the findings 
(Gay et al., 2009). This collective, multisite setting improves the generalizability and external validity of the findings with an end goal of informing future policy development (Creswell, 2007; Gay et al., 2009).

\section{Data Collection Tools}

Data collected in this study included written documentation such as course syllabi, program overview statements, general education overview statements, and policy statements from the sample of post-secondary institutions. Additionally, interviews occurred after a preliminary check of the collected data, and before a complete analysis of all written documentary data had taken place. These interviews were intended as a validity check in order to gain a deeper understanding concerning the environmental factors, policies, and institutional support for the teaching of media literacy in various settings, as well as to ensure as much data as possible was gathered from each participant site. The written documentary data did not require participant consent forms; however, consent forms were required for the 6 faculty that were interviewed. Institutional Review Board (IRB) approval was sought as being exempt, since the data collected was a combination of written documentation that did not contain specific, personalized information, as well as interviews with adults concerning non-sensitive topics. None of the institutions included in the documentary data collection sites were explicitly identified in the study. All interviewees, as well as each interviewee's institution, are anonymous; only the data collection category that each interviewee falls within is identified.

The written documentary data was collected through a combination of methods. First, the list of universities generated from Stuhlman and Silverblatt's (2007) most re- 
cent attempt at understanding a comprehensive view of all media literacy courses in institutions of higher education in the U.S. was referenced. Any publicly available syllabi that could be accessed from this list of universities were considered for possible data inclusion. If the syllabi met the design criteria and the institution was chosen, it was then placed within the appropriate data category based on the academic subject area within which the course is taught, as well as whether the institution is private or public. When one of these participant sites was chosen to be included in the data sample, appropriate accompanying publicly available documentation such as program overview statements, general education overview statements, and policy statements were collected and added to the data sample.

After this sample collection method had been exhausted, Internet search engine queries were conducted to identify further participant settings through publicly available syllabi that focused on, or included elements of, media literacy instruction. Identifying publicly available syllabi that met the requirements of this study, namely that a course does include elements of media literacy instruction, were subjective in nature and relied on the expertise of the researcher to correctly identify course syllabi that could be incorporated into the data sample. Once these additional settings were identified, the appropriate accompanying documentation such as program overview statements, general education overview statements, and policy statements were also added to the sample.

Finally, at this stage more data was required to ensure the designated number of samples to be collected for each data category, further Internet searches for courses listed on university websites that appeared to focus on, or included elements of, media literacy instruction but did not have publicly available syllabi were identified. The researcher 
communicated directly with faculty via email to request copies of the syllabi and any accompanying documents for those courses and their institutions. Once these syllabi were collected, reliance upon the expertise of the researcher to subjectively confirm the inclusion of media literacy instructional elements in each course was again required. If a course syllabus was deemed appropriate to be added to the data sample, all other accompanying documentation such as program overview statements, general education overview statements, and policy statements were added to the data sample.

At this stage, all 24 institutional participant sites were identified, and all of the design criteria requirements were met. An identification system was developed to organize and categorize all data sources; these identifiers were used throughout the duration of the study to assist in indicating sources of information. Because the initial data was based on pre-existing written documentation, this first data collection phase was not limited to external time constraints such as traditional semester lengths; the initial data collection ceased when all 24 sample participant settings were identified.

At the conclusion of the collection and preliminary analysis of the written documentary data, 6 validity-check interviews with faculty teaching these courses was conducted. It was anticipated there would be a combination of lesser developed settings with limited data available within its designated category, as well as more advanced cases where an institution might be perceived as being more successful in its implementation of media literacy instruction. A purposeful sampling strategy of 6 interviews (see Table 2) allowed for a manageable amount of interview data to be collected and analyzed, while still informing a better understanding of the implications of the research questions that are investigated in this study (Creswell, 2007). The intention of these interviews was to 
gain a deeper understanding of issues such as policy, institutional support, collaborative internal networks, and nuances of the participant settings that would not be able to be deduced solely from the analysis of written documentation.

\section{Table 2}

Faculty Participant Demographics

\begin{tabular}{lllll}
\hline & $\begin{array}{l}\text { Public 4-Year } \\
\text { Institutions }\end{array}$ & $\begin{array}{l}\text { Private 4-Year } \\
\text { Institutions }\end{array}$ & $\mathrm{n}$ & $(\%)$ \\
& & & & \\
\hline Sex & 0 & 1 & 1 & $17 \%$ \\
$\quad$ Male & 2 & 3 & 5 & $83 \%$ \\
$\quad$ Female & & & & \\
Race/Ethnicity & 0 & 0 & 0 & $0 \%$ \\
$\quad$ African American & 1 & 0 & 5 & $83 \%$ \\
$\quad$ Caucasian & 1 & 0 & 1 & $17 \%$ \\
Hispanic & 0 & 1 & 0 & $0 \%$ \\
Other & 1 & 1 & 2 & $33 \%$ \\
Years Teaching & 0 & 0 & 1 & $17 \%$ \\
$\quad 0-3$ & 0 & 0 & 0 & $0 \%$ \\
$3-5$ & 0 & 2 & 0 & $0 \%$ \\
$5-10$ & 1 & & 3 & $50 \%$ \\
$10-15$ & & & & \\
$15+$ & & & & \\
\hline
\end{tabular}

All interviews took place over software communication programs such as Skype or Zoom; there was never an expectation that the interviews would take place in a physical face-to-face setting. All interviews were recorded and transcribed for future coding and analysis. The interviews were semi structured, with a list of specific questions guiding each interview protocol, while still allowing for flexibility during the interview process based on answers from respondents (Merriam, 2009).

\section{Data Analysis}

As stated in the purpose of the study, this research began with an extensive review of the current literature. The ultimate objective of the scholarly review was to create a 
theoretical framework against which to evaluate the pedagogical approaches and learning outcome data collected and analyzed during the study. Scholarly reviews that are comprehensive in nature are ideal for determining instructional strategies and learning outcomes, which in turn can contribute to future policymaking (Harden, 2010; Sandelowski et al., 2006).

The research questions in this study were addressed through a combination of content analysis of the documentary data in order to discover the pedagogical approaches and learning outcomes of media literacy in higher education, as well as an ethnographic analysis of the interview data to discover how environmental factors, policy, and institutional support influence the teaching of media literacy in various settings (Merriam, 2009). The content analysis of the documentary data address R1 and R2. At the conclusion of this first phase of analysis, these findings, in addition to the findings from the qualitative follow-up interviews, inform R3.

Phase 1: Content Analysis. The first step of data analysis looked at the written documentation data of course syllabi, program overview statements, general education overview statements, and policy statements to answer R1 and R2. This data underwent content analysis to determine the presence of particular themes or words within the documents (Merriam, 2009). This first data analysis phase required cross-site analysis (Gay et al., 2009) that searched for generalizations in the pedagogical approaches and learning outcomes found in the data (Merriam, 2009). The coding rubric utilized during analysis was based on the various components of the theoretical framework created from the synthesis of literature in the scholarly review, titled the Participatory Ecological Framework of Media Literacy. 
All coding in this study was done by hand, without the assistance of computer software, to ensure all possible data findings were discovered. The researcher started the coding process for each piece of data by looking for the presence of the components of the Participatory Ecological Framework of Media Literacy, specifically the pedagogical approaches, media literacy concepts, and participatory methods of learning. As each of these components were identified, they were labeled by hand and color-coded based on an internal color-coding system created by the researcher. Once a piece of data was coded, the researcher then tabulated the results for the presence of each framework component that was present in the data and adding it to the full tally for all of the courses in this study.

This strategy of analysis allowed for accurate comparisons of the findings to the theoretical framework that was generated from the scholarly review (Merriam, 2009). Tabulation of the data also occurred, recording similar instances of occurrences among the data that corresponded to the pre-established data categories of the framework (Krippendorff, 2004). All efforts were made to explicitly state the interpretive standards utilized in discovering frequency data during analysis (Krippendorff, 2004). At the conclusion of the analysis of the written documentation data, the findings were organized to inform R1 and R2.

Phase 2: Ethnographic Analysis. At the conclusion of the collection and analysis of the documentary data that answered R1 and R2, 6 follow-up interviews took place with faculty members teaching 6 of these courses. The purpose of these interviews was to gain a deeper understanding of how issues such as politics, policy, institutional support, and internal culture are influencing the teaching of media literacy. Ethnographic 
analysis of the interview data allowed for further description and interpretation of issues outside of the pedagogical approaches and learning outcomes that could not be fully understood solely from content analysis of the documentary data (Merriam, 2009).

The interviews were semi structured, therefore a full recording and transcription of all conversations between the researcher and interviewees was conducted for analysis. The coding approach used for the analysis of the interview data was a combination of the pre-existing classification schema based on the theoretical framework created in the scholarly review, as well as additional categories that emerged from the data itself (Merriam, 2009). This extended classification system for the ethnographic analysis allowed for sociocultural data to emerge that would otherwise go unnoticed from the content analysis phase of the study (Merriam, 2009). Once the ethnographic analysis of the interview data was completed, the findings from both the documentary content analysis and the ethnographic interview analysis were considered collectively to answer R3. The validity and trustworthiness of this study was achieved by triangulating data from multiple sources through multiple methods of qualitative analysis (Creswell, 2014).

\section{Limitations, Assumptions, and Design Controls}

Considering that the most recent attempt at understanding the breadth of media literacy courses in higher education took place nearly a decade ago (Mihailidis, 2008), one limitation was that it could have been difficult to extend the findings of this study to a field of an unknown quantity. Specifically, in regard to the Stuhlman and Silverblatt (2007) study which attempted to account for all higher education media literacy courses being offered in the U.S. during the mid-2000s, the first data collection method in the present study utilized the list of universities generated from these findings. Their study is 
nearly a decade old, with no other scholars attempting similar investigations since; thus, the participant settings and findings from the Stuhlman and Silverblatt (2007) study had the potential to be outdated and ineffective for identifying appropriate participant sites at present. However, a design control was in place wherein multiple data collection methods were established; if issues arose from utilizing the findings from the Stuhlman and Silverblatt (2007) study, there were other mechanisms in place to ensure data collection would satisfy the intended criteria.

Assumptions can be made that the study will be found to be valid, reliable, and generalizable to similar contexts. The measures taken to ensure these assumptions are met included establishing a suitable sample size, collecting all relative documents in each setting, conducting follow-up interviews with a subset of faculty members from the institutional participants in order to gain a deeper understanding of the sociocultural issues in place, performing validity checks through the process of member checking and peer review, as well as practicing triangulation throughout the study (Gay et al., 2009; Merriam, 2009). However, by utilizing purposeful sampling of the participant sites, there is the possibility of researcher bias being present in the data, taking into account any possible intentions that were present during the data collection phase (Krippendorff, 2004).

A design control was put into place to address any possible partiality in site selection; though the study design designates sampling as purposeful in order to fulfill all required data category criteria, random sampling was simultaneously taking place through choosing participant sites from among a large population of any possible higher education institution teaching courses that focused on, or included elements of, media literacy instruction in the U.S. Other possible limitations, based on the qualitative methodologies, 
included issues of ambiguity of language, giving all phenomena equal importance, and philosophical assumptions of the researcher (Creswell, 2007). Next, the specific measures that were undertaken to ensure validity and reliability of the qualitative study are addressed.

Standards of Validation. In order to ensure the validity and reliability of this study, multiple techniques were employed in order to determine the accuracy of the findings. The validation strategies of data triangulation, peer review, member checking, and the clarification of bias were applied throughout this study. To achieve triangulation, the study made use of multiple participant settings, multiple methods of data collection, and multiple theoretical frameworks to provide various sources from which to corroborate findings (Creswell, 2007; Merriam, 2009). The peer review process was an external check of the data collection and analysis stages of the study by two external peers who were not members of the dissertation committee; the peer debriefing process included written documentation from both the peer reviewers and researcher in the form of written peer audits (Creswell, 2007).

To establish credibility, member checks were also conducted with as many of the participants as was feasible; a minimum of one member check per each category of data collection criteria was the goal, however only 6 member checks took place during this study. These member checks took place during preliminary analysis of the interview data as the findings emerged, in order to ensure that no misinterpretation of participant responses was taking place (Merriam, 2009).

\section{Definitions of Key Terms}

\section{Active Citizenship}


An engaged form of citizenship that is predominant among youth. This form of citizenship eschews the traditional duty-based view of citizenship with an emphasis on voting, paying taxes, and volunteering for military service in favor of one focused on participation (HeeYoung Kim, 2016). This active participation is fully integrated with today's digital media culture and is defined by civic engagement through free expression, advocacy, protest, and volunteering.

\section{Communication Models}

Communication models explain how pieces of information travel from an individual or group to another individual or group. These communication models have progressed over time, beginning with interpersonal communication between individuals and groups, and then changing with the introduction of mass communication in the last century (Silverblatt, 2014). Today's postmodern communication model is based on networked communication and interactive technologies.

\section{Digital Natives}

A popular, but contested, term used to define children raised in our current networked world reliant on digital technologies and media saturation. These students, who process instruction and knowledge based on their immersion in digital communication tools since childhood, are viewed as the native speakers of today's digital language. Growing up in this environment has changed the ways in which youth learn, with students often having difficulty excelling in educational settings based on teaching methods dependent on traditional communication models (boyd, 2014). A generational gap, based more on hearsay than empirical research, is often cited as causing conflict between digital natives and older generations. 


\section{Educational Technology}

An all-encompassing term that includes the approaches, components, and distribution of learning through or with the assistance of digital technologies. It is also seen as a practical, successful method of utilizing technology in a learning environment. Educational technology makes use of multiple types of media to assist in learning, including visual, textual, auditory, and other forms of communication.

\section{Literacy}

Traditionally viewed as the foundational ability to successfully write, speak, and listen. Today, the concept of literacy is defined as, "the ability to share meaning through symbol systems in order to fully participate in society" (Hobbs, 2010, p. 16). The most prevalent forms of literacy taking a critical approach to media texts today include media literacy, digital literacy, and information literacy. These terms are often viewed as being interrelated, with notable similarities and differences present among each.

\section{Media}

Media, the plural form of medium, is a term that simultaneously refers to the communication technologies used to transmit information, or media industries such as television, digital communication companies, and the publishing industry. The media is seen as being one of the strongest influences on the perceptions, beliefs, and attitudes of a society (Koltay, 2011).

\section{Media Literacy}

Defined as the ability to access media, understand and critically evaluate the different aspects contained therein, and create communications in a variety of contexts (Eu- 
ropean Union, 2007). The term media literacy consists of social and cognitive competencies that include critical thinking and analysis, creative media production, ethical understanding and engagement with media messages, and active participatory citizenship through the utilization of media technologies in a collaborative environment.

\section{Media Messages}

Media messages are the concepts contained in any type of media production, with information being transmitted in the form of words, visuals, sounds, or a combination thereof. All media messages are seen to contain some type of value or ideological component, as well as social and political implications (Silverblatt, 2014). An example of a media message could be a news broadcast, advertisement, social media post, or entertainment presentation.

\section{Neoliberalism}

Neoliberalism is a catch-all term that refers to liberal economic policies such as deregulation, privatization, and a hands-off approach of governmental interference with the private sector. Neoliberalism has emerged as the dominant perspective through which media policy has been structured and critiqued over the last three decades. Neoliberalism in regard to media policy frames the public as customers, commerce as the priority, and concentrated ownership through media conglomerates as the ideal structure (McChesney, 2003).

\section{Participatory Culture}

Participatory culture is seen as a culture in which citizens are able to not only consume information, but also produce their own content, contributing to the overall narrative of a culture. Recent developments in digital communication such as social media, 
mobile technologies, and ubiquitous Internet access have increasingly allowed citizens to work collaboratively, produce new creative works, and connect with others who share similar interests (Jenkins et al., 2009).

\section{Social Networking}

A term used synonymously with social media, this refers to the use of mobile communication technologies to create a personalized, open-ended interactive network (Silverblatt, 2014). Social networking platforms such as Facebook, Twitter, and Snapchat have become significant participants in mainstream media over the last decade. These social networking tools have redefined personal relationships and communication in cultures all over the globe, allowing for expanded participation of youth in politics and civic engagement.

\section{Text}

Similar to media messages, the term text is now understood to be any form of communication that utilizes symbols, language, still and moving images, graphic design, sound, music and interactivity, or in some combination of these components (Hobbs, 2010). Since all media presentations are produced and consumed within a cultural context, media texts can be seen to mirror the beliefs, values, and behaviors that define a culture (Silverblatt, 2014).

\section{Significance of the Study}

Media literacy is an innovative and emerging field that is in need of research concerning pedagogy and assessment (Mihailidis, 2008; Scharrer, 2002; Schilder et al., 2016). The results of this study provide insights into the disciplines where media literacy 
courses are taking place in post-secondary institutions, the teaching methods being implemented, and the learning outcomes for students. Conclusions from this study will contribute to educational policy by determining the pedagogical approaches and learning outcomes through a qualitative study of media literacy courses in higher education.

This evidence-based approach critically examines research findings to close the research-practice gap (Sandelowski et al., 2006). The additional integration of the literature synthesis informs policy in regard to media literacy education, similar to the approaches of countries in the European Union and Canada (Andersen et al., 2013; Lundgren, et all, 2013). The combination of the scholarly review of literature, the creation of a theoretical framework produced from a synthesis of the scholarly review, and the alignment of this new framework with the findings of the qualitative study represent a comprehensive and thorough examination of media literacy instruction in higher education institutions in the U.S. This study bridges the gap between knowledge and practice by critically examining media literacy education in post-secondary institutions with the aim of informing future educational policy.

\section{Scholarship}

Grounded in a synthesis of the scholarly review, this dissertation-in-practice proposes a new theoretical framework for media literacy instruction specific to higher education settings in the U.S. While most current media literacy instructional frameworks gravitate towards a focus on either critical analysis or creative production, the framework presented here instead takes a unified approach by integrating the components of analysis, reflection, and production into a single pedagogical practice. The introduction of a 
consolidated pedagogical framework aimed specifically at higher education settings contribute to the media literacy scholarship by addressing discrepancies in the instruction and learning outcomes for media literacy in post-secondary courses.

Additionally, aligning the data findings in this study with the new theoretical framework further scholarship in an understanding of the pedagogical approaches, core concepts, and participatory learning methods currently being utilized in media literacy instruction in higher education settings. The scholarly review of literature, the creation of the theoretical framework produced from a synthesis of the scholarly review, and the alignment of this new framework with the findings of the qualitative study represents a significant advancement in the scholarship of media literacy in higher education institutions in the U.S.

\section{Practice}

The dissertation-in-practice furthers the practice of media literacy instruction in higher education by offering a new theoretical framework that allows for the teaching of media literacy in multiple academic disciplines, in various post-secondary settings, and by faculty with varying levels of expertise in traditional media studies. The researchpractice gap is addressed by taking an evidence-based approach to the creation of the pedagogical framework and aligning it to the findings of the multisite qualitative study. By creating a new theoretical framework and testing it against findings from real-life post-secondary media literacy courses, it is possible to both improve current approaches to instruction and better realize intended learning outcomes. Additionally, Chapter 4 of this dissertation-in-practice, that of the Contribution to Practice, a policy brief intended 
for dissemination among higher education leaders and policymakers that provides evidence-based recommendations for the most efficient ways in which to include media literacy instruction in their curricula. Ultimately, this study bridges the gap between scholarship and practice by critically examining media literacy education in post-secondary institutions with a final aim of informing future educational policy.

\section{Summary}

Media literacy is a critical thinking competency that comprises access, analysis, evaluation, and communication of media messages (European Union, 2007; Hobbs, 2010). This new form of literacy is the foundation for effective knowledge production and civic engagement in our participatory culture (Jenkins et al., 2009; Mihailidis, 2008). However, media literacy education in post-secondary institutions lacks consistency in both pedagogy and learning outcomes (Mihailidis, 2008). Unlike most other developed nations, educational policy regarding media literacy in the U.S. is essentially absent.

This study informs future policy development for post-secondary media literacy education by determining the pedagogical approaches and learning outcomes of media literacy instruction in higher education institutions. This is accomplished through a comprehensive review of the literature, the creation of a theoretical framework, and a qualitative study whose findings are compared against the newly developed framework. The insights gained from this study will contribute to future university policy on media literacy education, ultimately bridging the gap between research and practice. 
MEDIA LITERACY IN HIGHER EDUCATION POLICY 34

Chapter Two: Practitioner Setting for the Study 


\section{Introduction}

Higher education institutions are in need of substantial research regarding the pedagogical approaches, learning outcomes, and assessment requirements of media literacy instruction (Mihailidis, 2008; Schilder et al., 2016). At this level of education, the subject itself can be viewed as being nearly nonexistent in a standardized form, especially when compared to its inclusion in elementary and secondary education. PK-12 media literacy instruction in the U.S. has the advantage of backing from national policy such as the Common Core State Standards. Compounding the issue is a lack of coherence in policy interchange between PK-12 and higher education due to organizational and financial disparities between the two systems (St. John et al., 2013). As a result of these inequalities in adoption and policy endorsement, identifying any type of standardized definition or learning outcomes for media literacy in higher education is problematic (Mihailidis, 2008).

An understanding of the breadth of the field is also difficult, with only a few limited studies taking place in the early and mid 2000's (Mihailidis, 2008; Stuhlman \& Silverblatt, 2007) that attempted to discover the total number of universities that included media literacy courses. Unfortunately, for a field that advances constantly in conjunction with shifts in culture, media, and technology, media literacy in post-secondary settings is deficient in most respects, scattered among varying disciplines to an unrecognized extent. Additionally, a comparison of media literacy education in the U.S. with the rest of the developed world presents a great imbalance.

As mentioned in the design of the study, the setting for this study consists of 24 courses from 24 different higher education institutions that focus on, or include elements 
of, media literacy instruction. With such a large number of individual settings for this study, it would be unsuitable to conduct individual organizational and leadership analyses for all participant settings. In the interest of adding the greatest potential value to this dissertation-in-practice, the practitioner setting is approached as viewing the field of media literacy education itself as the organization under study. This allowed for a more reasonable scope for this section, as well as provided a better understanding of media literacy and its related policy issues from both organizational and leadership contexts. By viewing media literacy education as the setting, some progress can be made in better addressing the lack of understanding in regard to the comprehensiveness of the field in higher education. Similarly, understanding the magnitude and nature of the field from an organizational and leadership standpoint can inform future policy analysis (Weimer, 2009).

This section begins by outlining the history of media literacy education in the U.S. Following the chronicle of media literacy's history, an organizational analysis of media literacy education takes place through employing the structural and political frames as outlined by Bolman and Deal (2008). Next, a leadership analysis focuses on the individuals who have been thought leaders in the field and are most responsible for the incorporation of media literacy instruction into the U.S. educational system. The section concludes with an explanation of the possible implications for future research in the field.

\section{History of the Organization}

Considering the goals of this dissertation-in-practice, as well as the large number of participant institutions that will constitute the data sample, a history of all the specific 
settings included in this study is problematic and unnecessary. Instead, as mentioned in the introduction to the practitioner setting for the study, this section views the field of media literacy education itself as the organization. Specifically, this view of media literacy from a holistic view of the field focuses on the history of the field in the U.S. educational system.

In the past, the field of media literacy could be characterized as being on the margins not only in the public consciousness, but also in relation to media education and production. Until the 1970s, any type of media literacy movement in the U.S. was disorganized and only taking place on small, local scales (Heins \& Cho, 2003). It has historically also focused on the needs of children and youth, with a significant demarcation between protectionist and empowerment schools of thought (Lunt \& Livingstone, 2012). The history of media literacy can be viewed as an organized effort by both the public and educators to develop an understanding of media communication in order to fully participate in a society where media plays an increasingly significant role (RobbGrieco, 2014). An understanding of this history is key for the forward momentum of the field to continue into the future.

The birth of media studies as a discipline in the U.S. dates back to the 1930 's, in a period where media was seen as a low culture, entertainment-focused pastime (Masterman, 1997). During this time, teachers were placed in the role of protectors, deciding which texts were appropriate for children and how language used in media messages was either authentic or inauthentic (Masterman, 1997; Nkana, 2014). This protectionist approach, as will be described in more detail later in this scholarly review, gives privilege to particular messages over others, substituting individual value judgements over critical 
analysis (Heins \& Cho, 2003). It wasn't until the late 1960s and early 1970s that educators stopped viewing the media as a corrupting influence, and instead integrated elements of popular culture into the classroom. During this time period, while teachers did not wholly abandon a protectionist stance, they did modify it to include elements of critical analysis (Masterman, 1997).

Media education in the 1960 s emphasized teaching about the media rather than through it, while encouraging students to develop critical thinking skills in regard to media messages (Goodman, 2003). In 1969 and 1972, respectively, major resolutions from the National Education Association and the Surgeon General's Advisory Committee on Television and Social Behavior recommended instruction in critical viewing in order to counteract assumed negative effects from the portrayal of media violence, thus shifting the focus of media education from film studies to television (Heins \& Cho, 2003; Nkana, 2014). In 1974, the first significant media literacy study took place at Harvard University, examining the effectiveness of media literacy education (Heins \& Cho, 2003). The study found that students who received formal media literacy instruction were more successful at critically analyzing television content, helping to solidify media literacy's efficacy (Heins \& Cho, 2003).

Though media literacy began to make inroads at the federal level through funding proposals during the Carter administration in the late 1970s (Heins \& Cho, 2003), by the early 1980s all federal interest stopped completely during the Reagan administration's focal shift to neoliberal education policies (Goodman, 2003). Conversely, during this same decade, while media literacy education was almost nonexistent in the U.S., other countries such as Canada, Australia, and the United Kingdom were making great inroads 
through the creation of national frameworks and media literacy education policies (Goodman, 2003; Silverblatt, 2014). The theoretical frameworks established internationally in the 1980s would later influence how media literacy was integrated back into U.S. classrooms in the 1990s (Goodman, 2003).

The 1990s saw a revitalization of media literacy in U.S. education. The 1992 Aspen Institute Leadership Forum on Media Literacy, which brought together 25 leaders in the field of media literacy, including significant Canadian representation, is seen as a symbolic event that led to the rejuvenation of media literacy education in the U.S. (Goodman, 2003; Heins \& Cho, 2003). This conference led to the definition of media literacy as the ability to access, analyze, evaluate, and communicate messages (Aufderheide, 1993), and is still viewed as the foundational base for all theoretical and practical frameworks developed since then. The Aspen conference identified data, recognition, theoretical frameworks, and collaboration as essential to the continued growth of the U.S. media literacy movement (Heins \& Cho, 2003); these needs would link representatives from public policy, educational reform, religious organizations, and the arts to promote the inclusion of media literacy in public education (Goodman, 2003). The conference proceedings also highlighted the importance of including media literacy elements in teacher education programs to ensure that future educators have the ability to incorporate the instruction into a diverse array of course subjects (Heins \& Cho, 2003).

The late 1990s can be highlighted by the continuous debate between protectionist and empowerment approaches (Lunt \& Livingstone, 2012). While protectionism is still utilized by parenting groups and child psychologists, during this period empowerment began to emerge as the preferred instructional model. Empowerment views media as giving 
the public an opportunity to fully participate in society through a critical understanding of media texts (Lunt \& Livingstone, 2012). These years also saw a renewed interest in media literacy education from the federal government, again primarily as a method for addressing the continued political issues regarding media violence (Heins \& Cho, 2003). Governmental interest in media literacy was fundamentally protectionist, while at the same time educators in the field were moving away from protectionism towards empowerment strategies. Meanwhile, research continued during these years that positively verified the effectiveness of formal media literacy education (Heins \& Cho, 2003).

In 2000, the National Association for Media Literacy Education published a refined definition of media literacy (Silverblatt, 2014) as, "empowering people to be both critical thinkers and creative producers of an increasingly wide range of messages using image, language, and sound" (National Association for Media Literacy Education, n.d.). This new definition shows the theoretical shift from worries of protecting youth from media violence to empowering them with the necessary skills to consume and create media through a lens of critical thinking. The National Communication Association developed formalized standards for PK-12 education during this period, which led to all fifty states adopting some form of media literacy competency standards for elementary and secondary students by the early 2000's (Yates, 2004). Media literacy's infiltration of higher education also took a significant step in 2006, when for the first time The College Board English Language Arts Framework recognized media literacy education as necessary to effectively participate in a global society (Silverblatt, 2014; The College Board, n.d.).

Recognizing the history of media literacy education in the U.S. distinguishes how media literacy has often been a response to constantly evolving technologies and social 
issues, keeping the field in a constant state of change (RobbGrieco, 2014). The ambition of briefly describing the history of the field is to show how disparate areas such as politics, popular culture, education, and technology have interacted over the past decades to produce media literacy education as it is understood today. Media literacy, however, tends to not focus on the past; rather it is concerned with the present, yet always with an eye to the future. This preoccupation with the present and future, while simultaneously qualified to shift as necessary based on current conditions, gives media literacy a strong foundation for continued growth (RobbGrieco, 2014). Now that the history of media literacy education in the U.S. has been established, the field will be analyzed from an organizational viewpoint utilizing the structural and political frames as defined by Bolman and Deal (2008).

\section{Organizational Analysis}

In viewing the field of media literacy as a whole for the practitioner setting of the study, the following organizational analyses will not be achieved in what would be considered a traditional fashion, or one in which an analysis would focus only on a single organization. Rather, the entire field is analyzed through the structural and political frames (Bolman \& Deal, 2008) in order to identify which specific issues would best inform the reframing process. Bolman and Deal (2008) define reframing as examining an organization from different viewpoints, or frames, in order to discover the relevant details that can best be used to turn information into actionable objectives. This process encourages continued organizational growth while simultaneously expanding what is considered to be common knowledge or assumptions about the organization (Bolman \& Deal, 2008). 
As mentioned previously, understanding the degree of the presence of media literacy in higher education institutions is challenging due to the limited number of studies that have attempted to acknowledge a holistic view of the field (Mihailidis, 2008). Rather than attempting to update past studies to comprehend the prevalence of media literacy in higher education, this organizational analysis instead utilizes frames as defined by Bolman and Deal (2008) to better inform future policy development through the reframing process. First, the field of media literacy is analyzed through the structural frame, followed by a secondary analysis applying the political frame.

\section{Structural Analysis}

Bolman and Deal's (2008) structural frame is based upon, "putting people in the right roles and relationships" (p. 47) in order to integrate individual objectives with overarching organizational goals. The structural frame assumes that (a) organizations are arranged in a particular manner in order to achieve objectives, (b) organizations are more efficient through the practical use of collective expertise, (c) successful collaboration requires coordination among a diverse group of individuals, (d) rational decisions must be placed in front of personal agendas for continued advancement, (e) a structural framework must be designed around an organization's present circumstances, and (f) obstacles arise from structural flaws (Bolman \& Deal, 2008). In a traditional organizational analysis, the structural frame would focus on the formally assigned roles and responsibilities within an organization. Since this analysis is considering the entire field of media literacy in higher education as the organization, these roles are innately decentralized among those experts working to further the acceptance of media literacy into the academe. In order to best address the policy needs of media literacy, this structural analysis focuses on 
the six assumptions listed above, rather than taking the approach of ensuring that assigned roles are being met within the organization due to the impractical scope of such a method.

It is easily recognizable that media literacy as a field was not established in a specific manner in order to achieve collective objectives. As was explained in the history of the organization section, media literacy has grown over time in a piecemeal fashion, and often in reaction to shifts in culture and technology. Because the field has developed out of personal intellectual viewpoints and circumstances, basic elements such as nomenclature and definition have still not been truly established or agreed upon (De Abreu, 2014; Hobbs, 2016). However, as an academic field it is understood that media literacy attempts to achieve a wide variety of goals, such as the ability to analyze and comprehend media messages, create original content, and enact civil action through ethical, socially responsible media use (Aufderheide, 1993; Hobbs, 2016).

The second and third assumptions of the structural frame are already established for the field of media literacy education. Specialization exists in the form of media literacy elements being taught in a multitude of academic disciplines (Silverblatt, 2014), as well as experts often specializing in various subsets of media literacy such as Internet culture, digital production, civic media, and the media representations of various socioeconomic groups (Hobbs, 2016). Again, with scholars teaching and researching from such a wide array of viewpoints under the umbrella of media literacy, diversity and specialization already exist within the field. The one aspect of these two assumptions that can be seen as insufficient would be the successful application of collaborative or collective ex- 
pertise, due to the decentralized view of recognizing an entire field of study as an organization. Shared policy guidelines that develop specific structures and processes specific to higher education settings could help alleviate issues of active collaboration among a farreaching collection of individuals (Kanter, 1994).

The overwhelming amount of diversity in media literacy education can be viewed as both positive and negative. On one hand, this diversity allows for true lateral coordination from an organizational position (Bolman \& Deal, 2008), creating a more flexible structure that can quickly and easily adjust in response to shifts in culture, technology, and communication (RobbGrieco, 2014). Simultaneously, these significant levels of specialization and diversification, which are often of a reactionary nature, are not congruent with the structural frame's grounding in strategic decisions being focused on long-term objectives through the utilization of allocated resources (Bolman \& Deal, 2008). In general, media literacy can be seen as possessing somewhat vague strategic goals that consist of complex, diverse, and always-changing actionable methods for achieving those goals.

The fourth assumption of the structural frame, which places the importance of rational decisions over personal agendas, is also problematic in the context of media literacy education. Because media literacy instruction is sporadic among higher education settings, often due to personal interest from specific faculty, its inclusion typically arises from personal agendas. That is not to infer that rational decision-making does not occur within the field, but it must be acknowledged that the scattered presence of media literacy courses in higher education is often due to individual passion rather than policy or administrative requirements. 
The fifth assumption, that a structural framework must be designed around an organization's present circumstances, is congruent with the current reality of viewing the field of media literacy as an organization in the context of this study. As has been mentioned previously, media literacy is flexible, as it is an always-shifting form of pedagogy. Since media literacy is a response to constant changes in culture, technology, and communication, it must always be ready for continued adjustments and restructuring (RobbGrieco, 2014). A perfect example is the shift from protectionist theoretical frameworks that traditionally anchored media literacy pedagogy, to the more current empowerment frameworks that are more compatible with today's media landscape; these changes will be explored further in the upcoming scholarly review. While this arrangement might not be compatible with Bolman and Deal's (2008) understanding of what constitutes structure, it reinforces the understanding of how media literacy education is structured.

The sixth and final assumption of the structural frame, which states that problems emerge from flaws in organizational structure (Bolman \& Deal, 2008), is an appropriate method through which to view media literacy education from an organizational stance. The objective of viewing the structure in this fashion is to mitigate issues through restructuring. Accomplishing this within a broad, complex, and admittedly liberally defined organization such as the field of media literacy education is difficult on account of many factors. First, the objectives of the field are still vaguely defined and not completely agreed-upon. Second, the amount of specialization and diversity within the field is so vast that it is challenging to fully appreciate. Third, the presence of the discipline on most university campuses is a result of individual agendas and interests. Finally, the field is flexible to an extreme, complicating any attempt to fully appreciate its structure. 


\section{Political Analysis}

The political frame has been chosen as a secondary analysis approach primarily due to the policy focus of this dissertation-in-practice. The relationship between political democracies, educational effectiveness, and policy work has long been established (Malen, 2011a). Policy development is political by nature, arising from systems of government that are aligned more closely with politics than rational decision-making (Swanson, 2009). Bolman and Deal (2008) designate the political frame for investigating organizations where separate individuals and coalitions possess their own independent agendas while contending with one another to gain what scarce resources are available. As has been made clear, this definition essentially describes the current state of media literacy education in U.S. higher education, with the exception that individuals and coalitions are not necessarily contending with one another; rather it has been shown that all the players have at least somewhat similar objectives. The field is scattered and tends to be local in scope, it is lacking in policy endorsement, and there are inconsistencies in pedagogical approaches, learning outcomes, and assessment requirements (Mihailidis, 2008; Schilder et al., 2016). Due to the political nature of these problems, the purpose of this section is to examine the various political aspects related to media literacy in postsecondary education settings.

Senge (2005) acknowledged that despite individual talent, complex organizations often have difficulty in maintaining stability or furthering growth, "because they are unable to pull their diverse functions and talents into a productive whole" (p. 441). Multiple internal and external factors can also influence an organization's success, such as competing interests for power and resources; perpetual conflict; differences in approaches and 
needs; and issues of compromise, bargaining, coalition-building, and negotiation (Bolman \& Deal, 2008). According to Bolman and Deal (2008), when viewing an organization through the political frame, complications often originate from power being too broadly dispersed. This is a perfect example of the current, broadly defined association of the media literacy discipline in higher education, with leaders in the field often working within their individual silos, while being limited in extending their influence and expertise outside of their narrow setting. The previous statement is not intended to belittle those dedicated individuals working diligently to further the continued advancement of media literacy in post-secondary education, but instead highlights how deficiencies in resources, coordination, and disbursement of political power can hinder the field.

Kanter (2005) identified easy access to resources and information, as well as successful collaboration, as being key in relation to power effectiveness. This is difficult to achieve in a field of study as sparsely dispersed as media literacy. However, opportunity does exist for influential leaders in the field to interact and form alliances; examples include the National Association of Media Literacy Education (NAMLE) in the U.S., and the Association for Media Literacy (AML) in Canada; there are literally hundreds of such organizations now present in the U.S. at local, regional, state, and national levels. Creating valuable environments such as these professional development networks can contribute to the political power of the field of media literacy education (Kanter, 2005).

While organizations such as NAMLE have achieved some success in regard to policy progress and overall awareness of the subject matter, the main obstacle is that these coalitions are transitory by definition and do not allow for the development of all forms of power as identified by French and Raven (2005). Reward and coercive power 
(French \& Raven, 2005) are of less concern to this organizational analysis based on the expansive approach being undertaken here, however the ability to demonstrate legitimate, expert, and referent power (French \& Raven, 2005) will be key if media literacy is to gain footing in U.S. educational policy. Expert power will be covered more in-depth in the following leadership analysis section; it is evident that thought leaders in the media literacy field are instinctively experts within their academic discipline.

In viewing the entire field of media literacy education in the context of this study, the fundamental deficiencies are a lack of both legitimate and referent power. Legitimate power requires the acceptance of a social structure (French \& Raven, 2005), and it has been demonstrated that post-secondary media literacy education lacks such structure due to the absence of national or state policies that are paradoxically present in PK-12 settings, for example. Policy development can assist in overcoming this obstacle by acting as a form of a legitimizing agent that is accepted by the individuals and alliances within the organization, as well as those external to the organization (French \& Raven, 2005). Referent power is based on worthiness or a shared identification (French \& Raven, 2005). As will be described in the scholarly review, there are multiple barriers to media literacy, many of which contribute to this lack of referent power. Considering the expansive premise of this organizational analysis, the most effective method for media literacy to achieve referent power would be to collectively utilize the field's high levels of expert power through policy development at the institutional level, demonstrating their extensive knowledge in a manner more easily understood by those outside of the field. Next, a leadership analysis of the practitioner setting is conducted through a combination of identifying the leading advocates and experts in media literacy education, as well as 
identifying the various leadership approaches they have employed to further advancement of the field within the U.S. educational system.

\section{Leadership Analysis}

This leadership analysis incorporates a brief examination of some of the thought leaders who have been fundamental to the advancement of media literacy education with a discussion of how various leadership approaches could successfully influence future policy development for media literacy in higher education settings. The term thought leader as used in the context of this leadership analysis refers to those individuals who have gone beyond simple expertise to demonstrating a body of scholarly work that demonstrates vast experience through a combination of their knowledge, influence, and innovation. Through an explanation of the travails these leaders have had to endure over recent decades to spread the doctrine of media literacy, one can more productively comprehend the current state of media literacy and possibilities for the future (RobbGrieco, 2014). The leaders to be addressed in this analysis are noticeably cited throughout this dissertation-in-practice.

The leaders considered in this section are not limited to the media literacy movement in the U.S.; international media literacy scholars and practitioners have had considerable impact on the field in the U.S. in regard to research, pedagogy, and policy development based on successes in their respective countries. However, identifying all of the thought leaders in the worldwide media literacy field is beyond the scope of this dissertation; the brief recognition of the leaders highlighted here is intended to demonstrate the leadership approaches they have utilized to successfully incorporate media literacy into the global education conversation. 
Similar to the field itself, leaders in media literacy education show a diverse array of understanding and perspectives from which they approach their work. These varying and sometimes conflicting approaches also demonstrate how personal intellectual viewpoints can lead to disagreements on foundational aspects such as theoretical frameworks and the terminologies used when describing media literacy (De Abreu, 2014; Hobbs, 2016). This has led to many coalitions forming within the field itself, which can be useful for the continued success of an activist pedagogy which contains divergent stakeholders (Hobbs, 2016). However, some cooperation among the leaders of these varying coalitions will be required if success is to be expected within the policy arena.

\section{Thought Leaders of Media Literacy}

Before establishing the thought leaders in the field of media literacy, some specific exclusions must first be justified. Educators such as Marshall McLuhan and Len Masterman, considered by most media educators as intellectual titans, were more focused on furthering media studies than media literacy specifically. McLuhan's text Understanding Media: The Extensions of Man (1964) and Masterman's book Teaching the Media (1985) are considered cornerstones of media education programs.

While both contain obvious components of media literacy pedagogy, it is due more to coincidence and the similarities among components of media literacy and media education than the intention of either scholar to give a focal point to the literacy component of media education. Furthermore, as was discussed in more detail in the history of the organization section, media literacy was still in its infancy at the time both works were published, with a somewhat agreed-upon definition not being established until the early 1990s. Although both authors continue to be enduring influences on media studies 
programs and the leaders to be discussed in this section, neither should necessarily be considered leaders of the media literacy education movement itself, but rather have inadvertently influenced the field through the close associations of their scholarly output.

Elizabeth Thoman. Early grassroots media literacy education in the U.S. in the 1960s and 1970s had a direct link to the Catholic church and other religious organizations, which spearheaded efforts in curriculum practices based on highlighting the disparities found in mass media representations and conservative Christian values (Heins \& Cho, 2003). Out of this religiously backed protectionist movement, Sister Elizabeth Thoman, a Catholic nun and graduate student at the University of Southern California in the late 1970s, began publishing a quarterly magazine called Media $\&$ Values, which was published from 1977 until 1993 (Heins \& Cho, 2003; RobbGrieco, 2014).

Media \& Values promoted a social analysis approach to media messages, incorporating the works of Paulo Freire with traditional Catholic teachings (Heins \& Cho, 2003). The publication was initially given financial backing by various Catholic organizations, with the Media Action Research Center, a multi-denominational religious organization, taking over the funding for the publication in 1983 (RobbGrieco, 2014). While it is important to recognize the religious foundations of the magazine, it should also be noted that the publication was groundbreaking in its scholarship and led to the creation of the Center for Media and Values, later renamed the Center for Media Literacy, which was the flagship non-profit organization promoting media literacy in the U.S. in the 1990s.

It is also noteworthy that the magazine, originally based on protectionist frameworks, shifted towards the empowerment paradigm in later years in conjunction with changes happening within the field. This openness to change displays Thoman's use of 
servant leadership (Northouse, 2013) to continue her work in promoting media literacy; with changes in the financial backing of her publication and organization, as well as changes in the way scholars view media literacy, she has always been attentive to the concerns of the field as a whole, rather than attempting to serve only her financial backers or the associated religious organizations, for example. She has proven that her goal is to serve the greater good of the organization, or broader community of media literacy education (Northouse, 2013). She has often been singled out among her own scholarly peers as being exceptional at developing and nurturing a community of media literacy stakeholders through her editorial work on the Media \& Values publication (Jolls, 2011). Thoman continues to be a thought leader in media literacy today through her work with the Center for Media Literacy in creating media literacy curriculum kits for educators and her continued scholarly research.

Barry Duncan. Barry Duncan's work in Canada, first originating from within his home province of Ontario, has cast a wide net of influence over worldwide media literacy education, and is noteworthy for a few reasons. Duncan helped to establish the Association for Media Literacy (AML) in 1978, which still remains the leading media literacy organization in Canada through the creation and open sharing of curriculum materials, theoretical framework development, and guiding national policy initiatives (Heins \& Cho, 2003). The Media Literacy Resource Guide, published by the AML in 1989, established eight basic concepts of media literacy that have since been cited frequently and incorporated into multiple other definitions and theoretical frameworks outside of Canada (Heins \& Cho, 2003). These concepts include: (a) media are constructs of reality, (b) media construct versions of reality, (c) audiences actively negotiate meaning, (d) media messages 
have economic implications, (e) media texts include value and ideological messages, (f) media texts have social and political implications, $(\mathrm{g})$ content and form are closely related, and (h) each medium has a unique aesthetic form (Association for Media Literacy, 2016).

In conjunction with the publication of the Guide in the late 1980s, Ontario was the first province to enact policy requiring media literacy to be included in its curriculum (Heins \& Cho, 2003). Following the lead of Ontario, all provinces have since developed policy ensuring media literacy's inclusion in secondary English courses, as well as some standalone classes in select provinces (Duncan \& Pungente, 2014). Media literacy has also become a required curriculum component in elementary education across all provinces (Andersen, 2013). Duncan's work with the AML has not only been responsible for the development of the frameworks and learning goals for all media literacy courses in Canada, it has also created the infrastructure necessary for the successful deployment of national policy initiatives.

It can be argued that Duncan represents transformational leadership through his long-term focus on the processes that affect and change people, or in this particular case, the Canadian education system (Northouse, 2013). While transformational leadership often focuses on instilling moral values and demonstrating ethical patterns for one's followers (Northouse, 2013), it is impossible to know if Duncan follows these guidelines personally and is a transformational leader among his direct followers. However, in the context that these organizational and leadership analyses are considering the field of media literacy as a whole, Duncan has demonstrated transformational leadership traits such as an idealized influence over pedagogy and learning outcomes, inspirational motivation for 
other provinces to follow the policy examples set forth in Ontario, and intellectual stimulation in allowing other media literacy instructors to creatively utilize the multiple frameworks introduced by the AML in contexts that work best in their particular classroom environments (Northouse, 2013).

Patricia Aufderheide. Patricia Aufderheide is most closely linked to being the author of the seminal report coming out of the 1992 Aspen Institute conference that established a definitive definition and basic framework for media literacy that has guided almost all other media literacy instruction in the U.S. since its creation (Heins \& Cho, 2003), and is cited as often as any scholar working in media literacy due to this publication. Before the conference, the field was inconsistent in its aims, often consisting of various passion projects enacted by individual faculty members, without any formal collaboration or uniformity among the players. Aufderheide, and thus the conference, identified four immediate needs for the field to be successful: data, publicity, infrastructure, and the creation of collaborative networks (Heins \& Cho, 2003).

Since the collaborative agreement of media literacy as being the ability to access, analyze, evaluate, and communicate media messages (Aufderheide, 1993), Aufderheide's definition has been considered the foundation by which almost all other definitions and frameworks are built upon. The pedagogical basis of this model is one of learning through hands-on participation in a process-driven, group environment (Aufderheide, 1993). While this definition is concise and contains the essential components of media literacy, it is limited to the extent that it is not adequate in our current climate of diversification and convergence of media (Livingstone, 2002; 2004). However, Aufderheide's successful utilization of expert power (French \& Raven, 2005) through her collaboration 
with scholars at the 1992 Aspen Institute conference displays how the situational leadership style can be effective within an ad-hoc organization with specifics demands in a singular, particular situation (Northouse, 2013).

Renee Hobbs. Renee Hobbs is considered one of the more recent pioneers of the media literacy movement in the U.S., being actively engaged in scholarly activity directly related to digital and media literacy since the early 1990s (Heins \& Cho, 2003). She was one of the early proponents of shifting the methods through which media literacy was taught from a protectionist approach, which had traditionally been the instructional method from media literacy's inception through the 1980 s, to the newly emerging empowerment theoretical frameworks. Empowerment frameworks view media not as obstacles that students must be guarded from, but rather as challenges which can be overcome through acquiring media literacy competencies (RobbGrieco \& Hobbs, 2013). Hobbs discovered that students at both PK-12 and post-secondary levels were becoming unresponsive to the notion of themselves being helpless victims of the influence of the media (Hobbs, 1998).

Much of Hobbs' early scholarly work focused on media literacy in PK-12 classrooms. She was involved in what was initially a controversial study which allowed Channel One, a for-profit news organization aimed at children, to broadcast in classrooms where she was conducting primary research to determine its effectiveness (Heins \& Cho, 2003). While the study showed that students exposed to the Channel One programming, and thus the most comprehensive media literacy curriculum, significantly outscored students not provided the full curriculum, issues of conflict of interest arose from Channel One's inclusion of two minutes of advertisements for every ten minutes of educational 
programming, as well as criticisms that an activist pedagogy such as media literacy was being commandeered by private interests in order to avert criticism of their products (Heins \& Cho, 2003). However, it should be noted that services similar to Channel One, such as CNN student news, have now become commonplace in American PK-12 classrooms since the conclusion of the study.

Since then, Hobbs has taught at the post-secondary level at Temple University and the University of Rhode Island, where she initiated the concept of the Media Education Lab for primary university resources to assist in advancing media literacy education through scholarship and community outreach (Jolls, 2011). However, Hobbs singles out the multidisciplinary nature of media literacy as being a hindrance to building a truly interdisciplinary program that addresses all areas and facets of media literacy education (Jolls, 2011). Hobbs, like Thoman, has demonstrated her servant leadership abilities through attempting to serve the greater good of the media literacy community through her research and outreach programs (Northouse, 2013). Hobbs demonstrated awareness (Northouse, 2013) in her Channel One study by understanding that by engaging directly with the media industry in order for the study to take place, it would be considered controversial among many of her peers, which could have personally negative effects. However, by being aware of the political environment at the time, she was able to view the situation from both her own perspective and the perspective of Channel One in the greater context of furthering media literacy research. In addition, she has demonstrated a commitment to the growth of others and an interest in community building (Northouse, 2013) through the creation and application of the Media Education Lab at the University of 
Rhode Island, with a mission to further media literacy education through community service and further research.

Buckingham and Livingstone. The scholars David Buckingham and Sonia Livingstone are being discussed together in this section due to their overlapping influence in media literacy policy development in the United Kingdom. Buckingham, outside of being one of the leading scholars of media literacy in Europe, has also been a consultant on policy issues for UNESCO, the United Nations, and the Office of Communications (Ofcom) in the U.K. (Jolls, 2011). Livingstone is similarly understood to be a thought leader in scholarly research and teaching, with a research focus on publics and youth in an ever-changing digital media environment (The London School of Economics and Political Science, 2016). Her outreach and policy work includes leading the Preparing for a Digital Future project, directing the EU Kids Online network which consists of thirtythree member countries in the European Union, and serving on the U.K.'s Council for Child Internet Safety (The London School of Economics and Political Science, 2016). Both of these scholars are some of the most oft-cited academics in the field of media literacy due to their decades of scholarship, outreach, and policy involvement in both the United Kingdom and the European Union.

The U.K. has a long history of media education, and while media literacy is not viewed there as a standalone subject, the outcome from a U.K.-based media education is an understanding of the, "process of teaching and learning about media" (Buckingham, 2003, p. 4). Both scholars consulted on the creation of the Ofcom 2003 Communications Act, which broadly deregulated the media industry in the U.K. while simultaneously ad- 
vocating for the promotion of media literacy in the education system (Wallis \& Buckingham, 2013). Unfortunately, since the inception of the 2003 Communications Act, digital literacy, which has a skills-based focus, has become the leading catalyst within the U.K. educational system, becoming the main context through which media literacy is referenced (Wallis \& Buckingham, 2013). In order to counter this approach, European Union policy proposed media literacy assessment tools for all of its member states (Lunt \& Livingstone, 2012); however, the continued success of this additional policy intervention is tenuous with the recent decision by U.K. voters to leave the European Union. Exacerbating the situation, even Buckingham (2016) himself has declared that media literacy as a policy initiative, one in which he was so closely involved in its development, is now essentially nonexistent.

Despite these recent setbacks, Buckingham and Livingstone have demonstrated authentic leadership by influencing national policy development through a combination of using their formal, expert power (French \& Raven, 2005) and by demonstrating relational transparency (Northouse, 2013) to create collaborative networks and organizations within the U.K. to further the media literacy agenda. Critical events are also pivotal for authentic leaders (Northouse, 2013); Buckingham's (2016) recent article lamenting the current stagnant nature of media literacy in policy discussions does not simply bemoan the end of a losing battle, rather in lieu of recent events on the European continent it challenges colleagues to discover new avenues to rejuvenate media literacy within U.K. media education curriculum. By taking a balanced approach to the constant changes connected to media literacy policy in the U.K., Buckingham and Livingstone have been able 
to continually analyze the current environment and consider public sentiment in order to engage in sound decision-making (Northouse, 2013).

Henry Jenkins. Over the past decade, Henry Jenkins has been considered by many to be the leading scholar in the U.S. in regard to concepts such as new media, media convergence, and participatory cultures. His groundbreaking book Convergence Culture, published in 2006, addressed the issue of how new, emerging forms of communication such as social media could best be utilized in conjunction with traditional forms of media such as print and television (Jenkins, 2006). Similarly, Jenkins was the lead author of a white paper for the MacArthur Foundation titled Confronting the Challenges of Participatory Culture: Media Education for the 21st Century that demonstrated the potentials of participatory culture in an age of new media technologies that can lead to creative expression and civic engagement (Jenkins et al., 2009). He also founded the Comparative Media Studies graduate program at MIT, one of the leading media studies programs in the U.S.

Jenkins' work in the field of media education has been nothing short of revolutionary; since the introduction of modern digital communication tools such as social media and mobile networking, it would be difficult to find any scholarly research on the topic without seeing his work referenced. While his research has not always specifically focused on media literacy, his work has been immensely influential to field and is the basis for many theoretical frameworks. Jenkins is an ideal example of a transformational leader through his continued influential scholarly work and charismatic vision (Northouse, 2013). The concepts he has introduced or popularized display transformational traits such as influencing others through setting a strong example, displaying high 
levels of competence in his area of expertise, clearly articulating what the goals of his research and others' should be, and has also communicated the high expectations he has for the continued relevance and elevated reputation of media literacy education in the U.S. (Northouse, 2013).

\section{Leadership Approaches to Influence Policy Development}

There are numerous possible leadership approaches that can lead to successful policy development in the area of media literacy. This leadership analysis, through a detailed review of some of the thought leaders of the field, has highlighted the leadership approaches of servant leadership, situational leadership, transformational leadership, and authentic leadership as being essential to future policy work. Servant leadership aims to serve the greater good of society through efficient communication, creating awareness of an issue, persuading others that change is necessary, possessing the ability to provide clearly understood goals to non-experts through conceptualization, and developing a community around which all stakeholders can rally (Northouse, 2013). Taking a situational approach to policy development is clearly suited to a field such as media literacy since it is in constant stages of modification in response to shifts in culture, technology, and communication (RobbGrieco, 2014). Situational leadership is best applied at the early stages of enacting strategy, and due to its fluid nature, applying a situational approach at the beginning stages of media literacy policy development is appropriate.

Transformational leadership combines charisma and innovation in an approach that, unlike most forms of leadership, is not based on power but rather engagement with others in order to motivate the field as a whole (Northouse, 2013). Clear articulation of goals and setting a strong example (Northouse, 2013) is especially crucial in policy work 
where practitioners and policymakers are often at odds and find difficulty in agreeing upon mutual objectives (Cohen \& Moffitt, 2011). Finally, authentic leaders can positively affect a complex issue such as policymaking through applying self-awareness and balanced processing, while at all times working transparently to ensure collaboration and support from others (Northouse, 2013). With the organizational and leadership analyses of media literacy education concluded, the next section discusses the future implications for research in the practitioner setting.

\section{Implications for Research in the Practitioner Setting}

While this organizational analysis utilized the structural and political frames (Bolman \& Deal, 2008) because those particular frames are best suited to analyzing media literacy from a policymaking standpoint, further analyses through the symbolic and human resource frames (Bolman \& Deal, 2008) would also be constructive for further leveraging media literacy's standing in higher education. Those in charge of policy tend to create or modify policy as a reaction to societal or political trends, often not taking into account research or suggestions from thought leaders (St. John et al., 2013). In order to reframe the policy discussion surrounding media literacy, successful collaboration among the stakeholders must be initiated in order to shift policy decisions so they are founded upon research and expertise rather than opinions and reactions to trends. It is expected that the results from this study, as well as the contributions to practice and scholarship, will assist in remedying this policy issue.

St. John et al. (2013) identified multiple approaches at the institutional level to address policy research with actionable objectives. St. John et al.'s (2013) critical-empirical approach will guide the upcoming contribution to practice section, however it is worth 
noting here the action inquiries that take place at the institutional level as examples of the implications for future research and practice at the settings for this study. In order to address both policy and organizational change, institutions must (a) use institutional data for the assessment and evaluation of upcoming policy challenges, (b) identify the most pressing social challenges in their settings, (c) organize collaborative groups to address these challenges through information audits, (d) organize an understanding of the challenges by considering possible internal and external solutions, (e) determine alternatives to the current situation and incorporate them into possible solutions, (f) run pilot tests in order to gather evidence and correctly evaluate the best policy options, and (g) reestablish policy intervention methods based on the collected data in order to align plans of action with institutional objectives (St. John et al., 2013). These action inquiries are intended to inform existing practices alongside future policy development with the least amount of disruption possible.

All of these future implications are based on enacting significant institutional change, which has the potential to impact multiple stakeholders at the practitioner settings. Implementing these policy changes will require calculated political action to ensure stakeholder approval. To be effective, actionable strategies will need to be developed with an understanding towards the specific setting and the most effective means of collaboration, influence, and communication particular to that institution (Bolman \& Deal, 2008).

Caffarella and Daffron's (2013) interactive model of program planning, used in conjunction with St. John et al.'s (2013) critical-empirical approach to institutional policy development, can help ensure a successful design and application of the institutional 
change to take place. The model includes eleven components to program planning, as well as five areas of foundational knowledge that must also be considered when enacting change (Caffarella \& Daffron, 2013). The five areas of foundational knowledge include technology, adult learning, cultural differences, relationship building, and power and interest (Caffarella \& Daffron, 2013). The eleven components to program planning consist of context, evaluation, details, learning transfer, marketing, instruction, goals and objectives, budgets, needs assessments, scheduling, and support (Caffarella \& Daffron, 2013).

Caffarella and Daffron (2013) point out that the model should be considered a guide rather than a master plan in actual practice and is an interactive method for thinking about the best ways to execute institutional change. An in-depth application of the interactive model of program planning (Caffarella \& Daffron, 2013), in combination with the critical-empirical approach to institutional policy development (St. John et al., 2013), will guide the upcoming contribution to practice section of this dissertation-in-practice. It is worth noting here though, as well since the implications from these two models will guide possible future change for the practitioner settings in this study.

\section{Summary}

A review of the history of media literacy education in the U.S. demonstrates how a small-scale movement originally based on the personal agendas of a handful of stakeholders has grown over the years to become an important and necessary instructional concern. Though the subject is presently being taught in some institutions of higher education, the breadth of the field is still unknown while disagreements surrounding the pedagogical approaches, learning outcomes, and proper assessment tools still abound (Mihailidis, 2008; Schilder et al., 2016). The political and structural frames (Bolman \& Deal, 
2008) are appropriate to understanding U.S. media literacy education as a collective organization because policy development is inherently political (Swanson, 2009), while the immense diversity and disparities found within the field requires some method for integrating all of the individual goals with the overarching organizational objectives. Leadership approaches such as servant leadership, situational leadership, transformational leadership, and authentic leadership (Northouse, 2013) can assist in helping to solve these organizational dilemmas through various methods. An appropriate utilization of all of these considerations in regard to policymaking will help media literacy shift from being on the fringes of post-secondary education to becoming a core component of the curriculum. 
Chapter Three: Scholarly Review for the Study 


\section{Introduction}

Media literacy is the viewpoint from which we access the media, critically evaluate its content, and create meanings from the images, words, and sounds in the messages we encounter (Aufderheide, 1993; European Union, 2007). Instruction in media literacy develops engaged, knowledgeable citizens who are acculturated to living in our mediasaturated society through lifelong use of their skills and experiences to construct their own meanings from media messages (Hobbs, 2010; National Association of Media Literacy Education, 2007). Practical understanding of this form of literacy is a basis for effective knowledge production and civic engagement in a democratic society.

Unlike other nations such as Canada, the United Kingdom, and countries in the European Union, the U.S. does not have a fully developed set of policies regarding media literacy education. This lack of policy support has contributed to discrepancies in the instruction and learning outcomes for media literacy in higher education. Mihailidis (2008) states that the, "inconsistencies in definition, use and adoption have ... hindered media literacy's ability to produce tangible and coherent learning outcomes for higher education" (p. 1). This lack of consistency manifests itself through a gap in the literature concerning media literacy pedagogy, assessment tools, and learning outcomes (Literat, 2014; Mihailidis, 2008; Schilder et al., 2016).

In the following sections of this scholarly review, an overview of the current media landscape and how students interact with media will be addressed, followed by a discussion of the foundations of media literacy. Next, the educational settings and theoretical frameworks through which media literacy instruction typically takes place will be examined. A review of media literacy educational policies in other countries will then be 
described and contrasted with the dichotomous policy environment in the U.S. Finally, a synthesis of the literature will lead to the creation of new theoretical framework intended to guide future media literacy instruction in higher education classrooms; this framework is also be used to compare and align the findings from the study. This scholarly review is comprehensive in order to best determine the current instructional strategies and learning outcomes for media literacy instruction in higher education, which will in turn contribute to future policymaking decisions (Harden, 2010; Sandelowski et al., 2006).

\section{Learning Media}

Media are a pervasive aspect of contemporary daily life and have arguably the most impact on our social discourse (Gaines, 2010). As technological innovations allow for new forms of media, the public adapts to them, thereby changing the ways in which people interact with one another and seek out information. These perpetual changes in communication forms and technology mean that the act of learning how to successfully interact with media is a lifelong process. A critical understanding of media communication establishes a greater understanding of the processes and effects of media and is essential to becoming a media literate individual (Gaines, 2010).

This is not a novel concept. Decades ago, Masterman (1985) argued why an education in media was an urgent priority. Masterman (1985) included media saturation, media's strong societal influence, the manufacture and dissemination of information, and the infiltration of the media into our democratic processes as some of the most important reasons for students to gain media literacy competencies. As well as the reasons above, possibly of greatest relevance is the need to educate our students to meet the demands of the future (Masterman, 1985). 
So how does one learn the media? Again, looking at the foundation of media education as presented by Masterman (1985), any type of media instruction, whether it be in media literacy, production, or media studies, requires an understanding of the core concepts, a proper theoretical framework, and a method of inquiry. This form of instruction takes place in a formal education setting, though it must be noted that informal learning is concurrently taking place outside of the classroom. The concept of combining informal knowledge with formal instruction, as will be furthered in later sections, is fundamental to successful media literacy instruction.

Media literacy educational settings, theoretical frameworks, and policy issues are examined in later parts of this scholarly review; this section explores the key principles of media literacy education, based on the development of media literacy over time, and the characteristics that make it unique among other new forms of literacy. Before considering the foundations of media literacy, however, an examination of the current media landscape in the U.S. will occur. It should be noted that this view of the current media environment is treated as a snapshot in time, as media and communication technologies are continually evolving.

\section{Current Media Landscape}

To understand the present media landscape is to recognize the strong influence that convergence has had upon all media industries in recent years. Media convergence occurs when distinct channels of communication that were once disconnected are now interwoven together so deeply that any unique characteristics that used to separate them are now completely nonexistent (Potter, 2013). Digital communication tools and the Internet, specifically, have led to a technological convergence among all media channels 
based on two-way communication. Interactive media is the most significant development to have grown out of this recent converged media environment. Unlike non-interactive media that is produced by professionals, interactive media requires that its audience also create content, interact with fellow audience members who are also content producers, and interact with media professionals (Potter, 2013).

Social media is a perfect example of a recently popularized interactive media platform. The three broad functions of social networking are social contact, the acquisition of information, and competition through some type of gaming (Potter, 2013). Social networking is unique in that it is an interactive media driven by interests and friendships (Potter, 2013). Researchers have found that communication within interactive media environments varies depending on the level of involvement; for instance, simple communication among friends is the least engaging type of interaction through social media (Ito et al., 2008). As youth grow up with interactive media being a key part of their daily lives, a new type of media literacy is required. This new literacy is based on the conscious use of informal language, subtle social norms for interactive engagement, and new types of media representation such as mashups, media blogs, and fan communities (Ito et al., 2008).

Twenty-first century literacy skills demand a new understanding of the traditional view of literacy; students must have multiple competencies because they are actively participating in a multi-literacy environment (Funk et al., 2016). This new form of literacy takes into account the diverse array of social situations where understanding is constructed and acted out by audience members who are now participants (HeeYoung Kim et al., 2016). However, today's students can still be segregated into groups of either active 
participants or passive consumers. Future ready learning: Reimagining the role of technology in education, the most recent national educational technology plan from the U.S. Department of Education, acknowledges that, "a digital use divide continues to exist between learners who are using technology in active, creative ways to support their learning and those who predominantly use technology for passive content consumption" (Office of Educational Technology, 2016, p. 5). This means that a student who simply uses social media to communicate with their friends does not necessarily possess a critical understanding of these new literacies.

In this section, a definition of today's learners being viewed as digital natives is first be established. Participatory cultures are next explained, as well as the role youth have within this culture as active digital citizens. Finally, the barriers that exist in hindering the acquisition of media literacy competencies are addressed.

Digital natives. The term digital native is often applied to today's youth because digital media and technology are so essential to their daily existence (Bates, 2015). These students have grown up in a networked culture with on-demand access to media through multiple platforms. The phrase itself was coined by Marc Pensky (2001) in his article Digital natives, digital immigrants. The term signifies a way to differentiate how today's youth, “think and process information fundamentally differently” (Pensky, 2001, p. 1) from past generations. Digital natives are seen as being the native speakers of more recent digital communication technologies such as the Internet and social media. It also positions anyone not born within the past two decades as being an immigrant, or outsider, to these technologies. The average mother who uses Facebook on a daily basis might disagree with this sentiment. 
The idea of a separation of generations or subcultures based on knowledge of communication technologies dates to the emergence of the Internet as a transforming cultural force. In John Perry Barlow's (1996) treatise, A declaration of the independence of cyberspace, he suggested the difference between past and future generations as those who lived within cyberspace and those who did not. His manifesto, while intended to cause provocation and highlight the independence inherent to the medium, was unintentionally accepted literally and further divided younger generations from older ones based on the assumption that natives possessed exceptional technological skills (boyd, 2014). The consequence of a term such as digital natives being understood without a critical analysis of its claims is that it simplifies the distribution of literacies among all youth, further complicating the notion that all students are prepared to meet the demands of the future (boyd, 2014), as extolled by Masterman (1985).

Digital communication technologies, specifically, have often been linked to a growing generational alienation (Silverblatt, 2014). As stated earlier, since today's youth have grown up with access to networked communication technologies, adults tend to assume that all youth are automatically literate in these technologies (boyd, 2014). Although digital media are innate to the average student's daily experience, it does not mean they have a natural understanding of the complexities for successfully navigating all of the communication technologies afforded to them. Despite now receiving more information than ever before, this exposure does not equate to a more informed citizenry (Papacharissi, 2010).

A decade ago, researchers were questioning whether a generational gap existed primarily between a new digital generation and their teachers (Buckingham et al., 2006), 
furthering any kind of digital inequality that existed in the public imagination. With the convergence of media and digital technologies since that time, however, it is now known that this gap is impermanent and participatory engagement with media is taking place among citizens of all ages (Jenkins et al., 2009; Jones \& Shao, 2011). It is understood, though, that different generations do tend to utilize different types of media in differing ways (Silverblatt, 2014).

Media play a key role in the socialization process, establishing and reinforcing social norms (Silverblatt, 2014). As children mature in an environment of constant connectedness, the media continue to have an ever-increasing role in their daily lives, dictating how they spend their time and conduct their social interactions (Livingstone, 2002). Our society is now inundated with information, and access to information continues to escalate at ever increasing rates. With the rise of new digital communication technologies, the problem has changed from attaining access to insulating ourselves from too much information. Other factors such as socioeconomic status and cultural context also play a role in the access, support, and knowledge of digital media (Bates, 2015; Morrell et al., 2013). Even the term digital native can be interpreted as loaded language, since there is no single generation of students entering the higher education system which can encapsulate the complex changes that are taking place with the continuous introduction of new technology and media distribution systems (Jones \& Shao, 2011).

Rather than blindly accepting assumptions regarding innate skills, educators and policymakers should instead focus on insuring students from varying backgrounds all have access to formal instruction on how to critically understand and navigate our net- 
worked culture. This new experience that views the public as equal participants and contributors to the media narrative is known as participatory culture. The convergence of both old and new media has created a participatory culture that allows for a greater diversity of media content, types of ownership and distribution, as well as a focal shift from one-way communication to interactive communication (Jenkins, 2006; Livingstone, 2002). The next section looks at the intersections of participatory communication and technology, as well as the potentials and pitfalls inherent with these constant communication technology interruptions.

Participatory cultures. The concept of participatory cultures is a neologism similar to the idea of digital natives. The term was popularized within a media education context in Jenkins et al.'s (2009) white paper for the MacArthur Foundation, Confronting the challenges of participatory culture: Media education for the 21st century. Participatory here means that citizens do not act as mere consumers of media and information, rather they also contribute to the culture by producing and distributing their own original content. Jenkins et al. (Jenkins et al., 2009) define a participatory culture as one with (a) low barriers to civic engagement and creative expression, (b) encouragement and support for individuals to create and share their content with others, (c) informal mentorship from experienced participants to beginners, (d) participants believing their contributions are significant, and (e) individuals feeling a social connection to others through their participation. Forms of participatory culture can include formal and informal partnerships, the creation of innovative media forms, collaborative problem solving, and creating the systems through which media content can circulate (Jenkins et al., 2009). 
One of the overarching objectives of understanding participatory culture through the lens of media education is to change the focus of any possible digital divide between generations from, "questions of technological access to those of opportunities for participation and the development of cultural competencies and social skills needed for full involvement" (Jenkins et al., 2009, p. xiii). This creates the opportunity for multiple potential benefits from different forms of participatory culture, such as destabilizing traditional media forms, opportunities for empowered civic engagement, a diversity of cultural expression, and creating new ways to teach and learn in the classroom (Jenkins et al., 2009; Mihailidis, 2014b). Having an audience member as an active participant in the producerconsumer relationship with traditional media means that the audience is now dictating how the media environment is shaped (Mihailidis, 2014b). Mihailidis (2014b) and others have also argued that as participation becomes more accessible and innate to the average citizen, the diversity of voices heard will continue to grow.

As stated previously, the assumption that students acquire these participation competencies naturally and on their own is incorrect. Jenkins et al. (2009) identified three crucial learning disparities that require pedagogical intervention: a participation gap, issues of transparency, and ethics concerns. A gap is present in reference to access of participatory media among youth, meaning that there are unequal opportunities to learn the knowledge and skills required to be a full cultural participant in the future (Jenkins et al., 2009). The problem of transparency relates to the challenges all younger generations face in recognizing biases and the ways in which media shape our societal norms (Jenkins et al., 2009). Finally, educators must ensure that students are properly socialized into the new emerging ethical standards that arise with the introduction of new models of 
communication and technologies where their private lives are now made public (Jenkins et al., 2009).

While not all members of a participatory culture must contribute, it is important that all members possess the necessary skills to be able to participate at any given time in the future. In a similar fashion to the idea of being a digital native, care must be taken to ensure that the ways in which we now define our youth and the society they are participating in are not defined by the technology itself, rather the interconnection between digital communication technologies, the citizens and cultural groups arising from them, and the participatory activities they allow. Next, an understanding of how our current participatory culture has redefined what it means to be an empowered citizen in a democratic society is established.

Active digital citizenship. One important aspect of the participatory culture we now find ourselves in is the seamless integration of digital media technologies into the daily lives of most people. This pervasive assimilation has led to, "the point that young citizens no longer organize their lives around information but instead organize information around their lives" (Mihailidis, 2014b, p. 18). Digital media have become so commonplace in our daily existence that they have become the primary way in which citizens engage in the construction of knowledge, communication with others, and the expression of individual creativity (Banaji \& Buckingham, 2013). With information overload now considered the norm, the need for media literacy competencies are more important than ever for students to understand how current issues affect democratic decision-making (Gaines, 2010). Recent studies also suggest that youth tend to eschew civic 
activities that are duty-oriented in favor of political engagement that is low-barrier and serves personal fulfillment (Powers et al., 2016).

These societal and technological shifts have led to a civic culture dependent on daily media integration, an understanding of community issues, and personal interaction with others in various participatory networks (Jenkins, 2006; Mihailidis, 2014b). To ensure the ideals of democracy, engaged citizens must acquire media literacy competencies that include the ability to communicate in a multitude of digital formats and use this understanding to better engage in their civic and political lives (Hobbs, 2010). Masterman (1985) specifically identified media education as crucial to a participatory democracy. The important relationships among democracy, literacy, and civic participation in everyday life demonstrates how crucial media literacy is in the 21 st century.

Current policy pressures of standardization and high-stakes testing are forcing public education in the U.S. to focus on global competition, to the detriment of democratic ideals (Funk et al., 2016). A key element of media literacy education is to strengthen civic participation through the teaching of media as a truly democratizing force, reasserting the promise of true democracy through an engaged, active citizenry. Both traditional and new media provide a means through which communicators focused on social change can reach their audience (Silverblatt, 2014). The recent growth in new digital platforms built on interactive communication through networked environments is allowing for collaborative interactions in a many-to-many form of communication not constrained by physical boundaries or demographic differences (Mihailidis, 2014b).

These new technologies have destabilized traditional media power structures, changing the ways in which citizens become informed about issues. Important examples 
of this shift include the Arab Spring, the Black Lives Matter movement, and recent upheavals in the U.S. political election process. Social change is now dominated by citizenled movements empowered by tools such as social media and mobile technologies (Fry et al., 2014; Mihailidis, 2014b). This new digital media culture is becoming central to civic society, reshaping how citizens interact with their communities and participate in civic culture in the 21 st century (Mihailidis, 2014b).

Where does media literacy education fit into this new collaborative environment? Mihailidis (2014b) suggests that by placing media literacy at the crux of the rationale for being a digitally engaged citizen, educators will understand why media literacy should, "be integrated into formal and informal education systems before it's too late" (p. 5). As advanced in the work of Paulo Freire (2010), media literacy education promotes an appreciation of the political nature of education and the understanding of literacy as a fundamental component of transformative learning (Funk et al., 2016). This ideal of today's empowered citizen can be understood through an activist media literacy education as promoted by Fry et al. (2014). This approach promotes the teaching of media literacy as a truly democratizing experience, questioning the status quo, and turning control back to participants and communities so they can take responsibility for engaging in information societies (Fry et al., 2014). A principal caveat to this methodology is the understanding that current students' involvement with civic and community activities is different than past generations due to their constant connectedness with digital communication technologies, thus educators cannot rely on past benchmarks of what defines political engagement (Powers et al., 2016). 
The activist media literacy education approach is based on the recognition of the political and economic power behind media institutions, critical analysis of media content, understanding biases and how these change media content in various ways, the realization of different media environments and how these social and mobile technologies shape how we communicate, and the ability to produce media content for civic good (Fry et al., 2014). This type of participatory education is based on more than just the consumption and understanding of media messages, but also the ability to produce and share one's own. This combination of critical inquiry and critical expression is at the core of media literacy's ability to accomplish an engaged digital citizenship (Hobbs, 2010; Mihailidis, 2014b).

Dewey (1938) emphasized the necessity of education for the existence of a strong democracy. The direct link between literacy and democracy means that students must have the abilities to critically understand meanings, engage with representations, and create their own content (HeeYoung Kim et al., 2016). Participatory approaches to media literacy education promote the current engagement-based concept of citizenship through critical interaction with media texts. With students now engaging in political action in differing ways from past generations (Powers et al., 2016), the consequences of media literacy's ability to provide students with the necessary tools to become knowledgeable citizens who endeavor to create a better society through their own personal expression are more important than ever. The next section considers the barriers that exist that can hinder the acquisition of media literacy competencies both inside and outside of the classroom. 
Barriers to media literacy. Mihailidis (2014b) identifies access as the origin of a successful media literacy education. Without simple access to media, a student cannot then move along the continuum of gaining media literacy competencies. In the context of media literacy, access here means access to both information and technology (Mihailidis, 2014b). Students should have access to (a) diverse sources of content, (b) diverse media texts, (c) adequate methods for seeking out and storing information, (d) the proper tools and skills for searching, producing, and using media texts, (e) the proper tools and skills for collaborating, distributing, and learning with media texts, (f) diverse audiences to receive their content, and (g) communities that further their lifelong learning and cultural participation (RobbGrieco \& Hobbs, 2013). Exposure to both a diverse array of messages and the tools that facilitate message creation and distribution should be seen as a fundamental right to citizens in a democratic society (Mihailidis, 2014b).

As will be examined thoroughly throughout this scholarly review, the most significant barrier to media literacy instruction is its lack of inclusion in the curriculum at all grade levels in the U.S. Many other nations have developed domestic policies that guide the teaching of media literacy in the classroom (Andersen et al., 2013; European Union, 2007; Lundgren et al., 2013). In the U.S., these policies are either nonexistent, or they include media literacy as a subset of other new literacies or are vaguely included in national standards (National Association for Media Literacy Education, n.d.). This lack of policy support has led to inconsistencies in an understanding of what media literacy actually is among educators, in its learning outcomes, and ultimately in its adoption (Mihailidis, 2008). Additionally, media literacy education instruction and research often focuses on PK-12 education, with a lack of study for higher education settings (Koltay, 
2011; Schmidt, 2013). Exacerbating these issues are the recent policy preoccupations with standardized tests, which have moved fundamental media literacy components such as critical thinking, project-based learning, and group work to the fringes of American classrooms (Kellner \& Share, 2005).

Along with the lack of access to media literacy instruction for students, a lack of resources for educators also hinders its ubiquitousness in the curriculum. Post-secondary educators, for instance, cite a lack of university-provided resources to digital tools and media as being a significant hindrance (VideoBlocks EDU, 2015). This lack of resources is often a byproduct of administrative ignorance, with many administrators confusing media literacy with mere technology access (Considine et al., 2014). Media literacy is also often taught in a cross-curricular environment, which means that many faculty are not properly trained in media studies, making teaching and assessment especially difficult (Schilder et al., 2016).

Contrarily, recent research has suggested that digital media and communication technologies are now commonplace in the classroom; the issue then becomes not whether they should be integrated into the curriculum, but rather how instructors can best incorporate digital and media literacies into their preexisting courses (VideoBlocks EDU, 2015). As schools and universities have continued to be cautious in gaining the resources necessary for media literacy to be included in their courses, students are then forced to gain media literacy skills outside of the classroom and without formal instruction from their teachers (Buckingham, 2007; Jenkins et al., 2009). Faculty attempting to include media literacy pedagogy into their classrooms also have to navigate inconsistent and varying policies from one location to the next (Considine et al., 2014). 
Cultural insulation is another significant barrier to media literacy and happens mostly outside of the classroom. Cultural insulation occurs when individuals selectively expose themselves only to media that reflect their personal interests and ideology, remaining willfully ignorant and indifferent to alternative viewpoints (Silverblatt, 2014). This insulation threatens the very ideals upon which culture is based, preventing common values and experiences to be shared among citizens. The ability for individuals to culturally insulate themselves has become easier with continued media deregulation which creates further narrowcasting and decreased diversification of content (Copps et al., 2005). More recent digital communications such as social media have also become exceptionally specialized as to further assist in this insulation, allowing for a microcasting system that caters to the individual tastes of single users (Silverblatt, 2014). One way to combat cultural insulation is through content analysis, which is a technique that looks for patterns in media messages. This methodology is a component of some media literacy pedagogical frameworks and is useful for identifying potential biases and underlying ideologies present in various texts (Silverblatt, 2014). Now that today's media landscape has been established, the next section introduces the foundations upon which media literacy is built by looking at its history, what distinguishes it from the other new literacies, and the key principles to this pedagogy.

\section{Foundations of Media Literacy}

In order to understand the instructional frameworks for media literacy, as well as the associated policy issues, a foundation of how and why media literacy developed as a pedagogical practice must first be addressed. This field can be viewed as always shifting, a type of living pedagogy. Because media literacy is a response to constant changes in 
culture, technology, and communication, it must be fluid and always ready for further modification (RobbGrieco, 2014). However, it is important to distinguish the media and educational infrastructures upon which media literacy is based. This section identifies the foundations of media literacy by discussing the different models of communication, what distinguishes media literacy from other new forms of literacy, as well as the key principles inherent to all theoretical frameworks.

Communication models. Communication models explain how different pieces of information travel from one individual or group to another. These different models have shifted over time, from the interpersonal communication between individuals to the introduction of mass communication in the last century, to today's postmodern model based on digital communication and interactive technologies (Silverblatt, 2014). Interpersonal communication consists of the communicator, message, channel, and audience. In this model, communication tends to be from one entity to another, with information being shared through spoken means to another party (Silverblatt, 2014). It is also important to denote the ways the elements in these different communication models shift over time; the interpersonal model consists of a communicator sharing a message through a channel to their audience.

In the mass communication model, the channel is served by different mediums such as text, visuals, sounds, or a combination of all three. With the rise of mass media institutions in the early $20^{\text {th }}$ century, these channels most often took the forms of newspapers, radio, and television. In the mass communication model, the channel has now taken the primary role in communication (McLuhan, 1964). With mass media gaining dominance, this model is represented by the channel serving as the first line in information 
sharing among large groups of people separated in both place and time from the media channel (Silverblatt, 2014).

During this era of the mass communication model, media literacy first emerged as a mechanism for protecting citizens from any possible negative or corrupting influences of the media (Nkana \& Silverblatt, 2014). Early media literacy education frameworks reflect this protectionist viewpoint based on the mass communication model (Buckingham, 2003; Nkana \& Silverblatt, 2014), the details of which will be covered in a later section of this scholarly review. The emergence of the Internet saw a significant shift in both communication models and the approaches to teaching media literacy.

Digital communication ushered in another change in communication models, from a mass communication model to a postmodern communication model based on the interactive participation of the audience. Digital communication is defined as interactive correspondence between an initiator and a receiver, with messages being transmitted through digital technologies that mimic human communication patterns (Silverblatt, 2014). This new model gives much more power to the audience and less to the channel; audience members are now able to bypass what is now perceived as traditional media outlets to become their own content curators and creators.

Web 2.0 technologies such as social media have been the strongest arbiter of this cultural shift, creating a participatory culture based on digital networking as being the most effective way of sharing information to and among citizens (Jenkins, 2006). In conjunction with this revision in communication models, the frameworks through which media literacy is taught also underwent a significant change, deviating from a protectionist 
model to one of empowerment, better reflecting the changes in communication and related technologies. This shift in theoretical frameworks owes to the influence of Dewey (1938), who believed that education was essential to societal progress; the specifics of the advancement of empowerment frameworks are examined in a future section as well. Now that the structure of media communication has been established, the characteristics that set media literacy apart from other new literacies is described, as well as how these literacies developed independently from one another.

Different forms of literacy. The traditional view of literacy as having a knowledge of letters and text, then turning that knowledge into the ability to read and write, now applies only to the print medium. Our society is now in a post-typographic age, with digital media allowing for information to be available in a variety of formats in once-unimaginable quantities (Koltay, 2011; Lankshear \& Knobel, 2008). Literacy now contains components of visual, electronic, and digital forms of communication, which are all tied to technology and culture (Koltay, 2011).

There is now what can be defined as a multi-literacy movement in education, which attempts to address all of the needs of 21 st century students (Van Heertum \& Share, 2006). Multi-literacy as a pedagogical approach arose from the availability of diverse new forms of digital communication and the advancement of globalization (Cope \& Kalantzis, 2009). This new multi-literacy environment includes media literacy, visual literacy, information literacy, digital literacy, and technology literacy, among others (Koltay, 2011). Visual literacy is now most often seen as a component of media literacy, while technology literacy has been folded into digital literacy. The three most prevalent segments of multi-literacy education today are media literacy, information literacy, and 
digital literacy (Koltay, 2011). These literacies have also more recently been combined into a catch-all competency known as new media literacy (Chen et al., 2011).

With these multiple forms arises significant confusion and discrepancies among scholars. The definition of media literacy has been established in this scholarly review, yet there are continued definitional variations that have created problems for digital and information literacy, and thus through vague connections for media literacy as well (Koltay, 2011). For the purposes of this study, information literacy is defined as the ability to select and retrieve information in any setting, especially in schools, libraries, and the workplace (Koltay, 2011). This literacy also emphasizes cognition, critical thinking, and very specific steps to successfully locate information in a variety of contexts (Koltay, 2011). It is most often tied to library sciences and the search for information online and in library databases.

While there are, at best, cursory similarities between media literacy and information literacy, digital literacy is much more closely aligned to media literacy, thus creating more inconsistencies when the two forms are mentioned together. Digital literacy was first introduced in the 1990s as a way to signify those who could or could not interact with hypertext on the Internet (Koltay, 2011), or rather the ability to successfully utilize information from specifically digital sources (Bawden et al., 2008). The four core components of digital literacy include (a) internet searches, (b) hypertext navigation, (c) assembly of knowledge, and (d) evaluation of content (Bawden et al., 2008). Digital literacy has a wide and varying definition, as well as a direct relationship with media. Martin (2006) identifies digital literacy as: 
The awareness, attitude and ability of individuals to appropriately use digital tools and facilities to identify, access, manage, integrate, evaluate, analyze and synthesize digital resources, construct new knowledge, create media expressions, and communicate with others, in the context of specific life situations, in order to enable constructive social action; and to reflect upon this process (p. 19).

Key terms such as evaluate, analyze, create, and communicate stand out in this definition because of their direct link to various definitions of media literacy. A consensus has also formed around the notion that digital literacy must include some form of media (Rosenfeld et al., 2016). Because of these similarities, some scholars will include media literacy as a component of digital literacy, or vice versa. To further complicate matters, digital media literacy is a more recent term that is sometimes viewed as a subset of media literacy. Digital media literacy refers to a wide range of interactive media devices, including social media, the Internet, mobile communication, and still and video production technologies (Silverblatt, 2014).

For the purposes of this study, the three most prevalent new literacies of information literacy, digital literacy, and media literacy are all viewed as distinct literacy approaches in our current multi-literacy education environment. Bawden (2008) designates that there is no single literacy that could possibly encompass all of the necessary life skills required for citizens to successfully navigate the constantly changing circumstances of our digital information environment. Because of this, media literacy must be able to stand on its own as a specific form of literacy within education, or at a minimum as one 
of the key components of a multi-literacy education (Koltay, 2011). Next, the key principles that are core to all definitions and frameworks of media literacy education are explained.

Key principles of media literacy education. Media literacy can be generally defined as being a critical thinking skill that helps citizens to develop the ways in which to analyze, discuss, and produce media texts while simultaneously understanding the impact media has on society (Silverblatt, 2014). These competencies help people to intelligently use and construct media, view media messages with skepticism, and critically understand different media forms and the effects and uses that come with them (Kellner \& Share, 2005). Generally speaking, this pedagogy incorporates elements from a combination of media studies, constructivist learning, and cultural studies. While there are numerous frameworks available through which to teach media literacy curriculum, the core concepts as adopted by both the National Association of Media Literacy Education (NAMLE) and the Center for Media Literacy (CML) guide in identifying the key principles included in all definitions or frameworks regarding media literacy education in the U.S.

The six core principles of media literacy as identified by NAMLE provide a framework that focus on critical thinking, personal involvement, and cultural socialization (Mihailidis, 2014b). The core principles state that media literacy education (a) requires active inquiry and critical thinking regarding media texts, (b) expands the definition of literacy to include media, (c) reinforces skills for learners of all ages, (d) develops engaged and informed citizens that are necessary for a democratic society, (e) observes 
media as an important part of culture and socialization, and (f) maintains that people understand meanings based on their personal skills, beliefs, and experiences (National Association for Media Literacy Education, 2007). These principles allow the field of media literacy education to include what is taught as well as how it is taught, distinguishing the core principles as a foundation of media literacy education, and not solely media literacy itself (HeeYoung Kim et al., 2016).

The five concepts as defined by the CML position media literacy education as being equally important to both the creation and intent of media messages (Mihailidis, 2014b). Based on these core concepts, Thoman and Jolls (2003) define media literacy as having:

Basic higher-order critical and creative thinking skills, e.g. knowing how to identify key concepts, how to make connections between multiple ideas, how to ask pertinent questions, formulate a response, identify fallacies, that form the very foundation of both intellectual freedom and the exercising of full citizenship in a democratic society. (p. 6)

The CML core concepts include (a) authorship, (b) format, (c) audience, (d) content, and (e) purpose (Thoman \& Jolls, 2003). Authorship means that all media messages are constructed, while format views media messages as being constructed using a creative process with unique rules (Thoman \& Jolls, 2003). The concept of audience means that different individuals experience similar media messages in varying ways (Thoman \& Jolls, 2003). Finally, content views media as having an embedded value judgement or point of view, while purpose understands all messages as having some aim to gain power or profit (Thoman \& Jolls, 2003). 
Taking into account the key principles of media literacy education from both NAMLE and the CML, as well as Aufderheirde's (1993) definition of media literacy as being the ability to access, analyze, evaluate, and communicate messages, a solid foundation can be established that informs all modern theoretical frameworks. Comprehensively, media literacy can be viewed as promoting critical thinking to empower citizens to make informed decisions in response to media messages (Silverblatt, 2014). Critical thinking as a pedagogical component appears regularly in regard to media literacy, as is evidenced throughout this study. This critical thinking is dependent on the process of inquiry, or the ability to utilize one's critical thinking skills to question, analyze, and ultimately understand issues presented through the media (Silverblatt, 2014). Keeping in mind these key principles through the lens of critical thinking will allow a full understanding of the base informing media literacy education. This instruction, rather than being based on replacing student perspectives with the instructor's, is founded upon the sharing of competencies that allow students to critically question and analyze media messages for themselves (Silverblatt, 2014).

\section{Conclusion}

Our media landscape is currently at a compelling juncture, offering unique opportunities and challenges. Today's students have matured in an environment of ubiquitous access to information and media through constantly changing digital communication technologies. The practices emerging from our new participatory culture are allowing for all voices to now be heard. Participatory approaches to media literacy further an engagement-based perception of citizenship through a critical interaction with media messages. 
Successful media literacy instruction provides students with the necessary tools to become knowledgeable citizens who endeavor to create a better society through their own personal expression. Key to achieving these outcomes is to overcome possible barriers to a media literacy education such as issues of access and inadequate teacher resources.

Media literacy initially developed as a method for protecting vulnerable members of our society from any perceived negative effects from the media. Not until the early 1990s did the media literacy movement reach a moderate consensus in its definition, educational approaches, and core components; during this time the field shifted its focus to empowering citizens to contribute their respective voices to society through critical media analysis and creative interactions. Media literacy also emerged as one among many new forms of literacy that has updated the definition to include other forms of communication aside from print. The story of media literacy is one of educators and the public developing an understanding of media communication that allows everyone to fully participate in a society where media plays an increasingly significant role. With the current status of media's role within American society and an understanding of the background of media literacy firmly established, this scholarly review next explores the frameworks and settings of media literacy instruction in the U.S.

\section{Media Literacy Education}

The inclusion of media learning activities in the classroom allows for students to both explore new media forms and participate in digital cultures (RobbGrieco \& Hobbs, 2013). This form of learning emphasizes a focus on the learners engaging with media through collaboration, play, problem solving, critical thinking, and socialization with their peers (Jenkins et al., 2009); National Association for Media Literacy Education, 
2014; RobbGrieco \& Hobbs, 2013). However, schools have been slow to incorporate media literacy instruction into their curricula, hindering students from being able to practice their new media literacies and skills in the classroom (Buckingham, 2007; Jenkins et al., 2009), and instead are expected to gain media literacy competencies outside of the classroom without formal instruction. While media and digital communication technologies are in a constant state of development and becoming increasingly integral to daily life, media literacy instruction has not matched this pace and remains on the margins of the education system.

Government and school policies currently maintain that technological, information, and digital literacies are of more importance in the classroom than media literacy. These forms of literacy are based on the efficient implementation of particular skills and have only cursory similarities to media literacy (Buckingham, 2009; National Association for Media Literacy Education, 2014). While there is no single literacy that encompasses all of the proficiencies required for successful navigation in today's society, media literacy's inclusion in some form of multi-literacy education is indispensable as our interaction with media continues to grow on a daily basis. A more in-depth investigation of the implications of these multimodal literacy policy decisions appears in a later section of this scholarly review.

Since schools in the U.S. have decided that media literacy education should be kept on the periphery of the classroom, the responsibility has often fallen to after-school and local community programs to teach youth how to navigate this new participatory culture (Jenkins et al., 2009). These programs are most often based on the notion that youth media production is the most effective method for teaching media literacy (Van Heertum 
\& Share, 2006), which is often viewed only as a component of the more robust theoretical frameworks that guide instruction in formal settings. The potential for media literacy to empower youth as active citizens also exists (Buckingham, 2007), however, it is difficult for learners to decipher contrasting media messages such as factual information, content manipulation, and advertising on their own (Seiter, 2007). Media literacy is, in essence, a cultural competence that should be formally taught to students in a collaborative, social classroom environment (Jenkins et al., 2009).

The importance of media literacy education should not be viewed as a burden of an additional subject that has to be added to an already overcrowded curriculum; rather this literacy emphasizes teaching students how to access and critically evaluate meanings from the images, words, and sounds found in the daily media messages they encounter (Aufderheide, 1993; Potter, 2004). Unfortunately, with technology and media communications in a constant state of flux, it is only with varying levels of success that teachers have been able to translate the new realities of the intersection of culture, media, and technology to their students (Van Heertum \& Share, 2006). This section of the scholarly review examines the ways in which formal media literacy instruction takes place in American education, with a more in-depth view of the different curriculum approaches utilized within a higher education setting. This is followed by an investigation into the development of prominent media literacy frameworks over recent decades, as well as the specific pedagogical approaches contained therein.

\section{Settings of Media Literacy Education}

The teaching of media literacy in a formal classroom setting guides students to investigate how media impacts both our society as a whole and themselves as individuals. 
This instruction teaches critical-thinking skills and helps students to become more cognizant of the impact that various media have on their thoughts and actions. This study posits that all students would benefit greatly from participating in media literacy coursework. However, due to the nature of media literacy courses, it can be difficult to convince administrators and policymakers that this curriculum is necessary. Media literacy as a subject is built around the consumption and discussion of media, which equals a great deal of screen time and media exposure for students both inside and outside the classroom. Despite this, the reality is that most students are already engaging with media for significant periods of time each day; media is the predominant place where they exist and learn.

One of the oldest debates among media literacy educators involves the merit of stand-alone media literacy courses versus an integrated approach in which media literacy principles are incorporated into other established disciplines (Silverblatt, 2014). Historically in the U.S., media literacy instruction has been incorporated into language arts, health education, and social studies courses, while only occasionally as a distinct course (Heins \& Cho, 2003). As will be seen, there are positives and negatives to both approaches in all settings of media literacy instruction. Additionally, though not a focus of this study, it should be noted that in the U.S. media literacy is often taught in after-school, community, and lifelong learning programs as a stopgap measure to address the lack of instruction in formal education environments (Jenkins et al., 2009; RobbGrieco \& Hobbs, 2013). The next sections of this scholarly review examines the various ways in which media literacy is taught in PK-12 and higher education settings.

PK-12 settings. In the U.S., formal media literacy instruction has traditionally been most prevalent in elementary and secondary education. Though this study focuses 
on media literacy in a higher education context, it is important to recognize both the positive strides that media literacy has taken in recent years in PK-12 settings, as well any previous media literacy instruction university students could have been exposed to earlier in their educational careers (Schmidt, 2013). An examination of media literacy in PK-12 education requires an understanding of the different subject areas that include a component of this instruction, as well as how it connects with the Common Core State Standards (CCSS).

Media literacy as a standalone subject in PK-12 classrooms is nearly nonexistent in the U.S.; instead, it is integrated into multiple subjects throughout the curriculum. The most prominent subject to include elements of media literacy instruction is English language arts. Literacy is the foundation of English language classes, and many teachers use media literacy as a method for connecting print to critical thinking through engagement with the media (RobbGrieco \& Hobbs, 2013). English teachers also often incorporate elements of information literacy and critical media literacy to connect media and technologies that students are already familiar with to current events or themes of social justice (RobbGrieco \& Hobbs, 2013). Media literacy also has the strongest ties to the English Language Arts standards present in the CCSS. Other PK-12 subject areas that often incorporate elements of media literacy instruction are social studies, journalism, science, technology, and health studies (Kubey \& Baker, 1999; RobbGrieco \& Hobbs, 2013). Media production courses, though not usually prerequisite subjects, will decidedly include media literacy as a core component of the course. At the elementary and secondary level, media production courses focus on giving students a voice through creative production projects, as well as addressing issues of representation of different groups in their 
work (RobbGrieco \& Hobbs, 2013), giving many of these classes a predisposition towards critical media literacy frameworks.

As stated earlier in the discussion of the history of media literacy education in the U.S., the National Communication Association developed formalized standards for PK12 educators in the late 1990s, which led to the adoption of competency standards in all fifty states by the early 2000s (Yates, 2004). The recommendations for initiating these standards were voluntary (Yates, 2004), however, and were not part of any standardized, national program such as the current Common Core standards. More recently, with the adoption of the CCSS, media literacy now has multiple direct and indirect connections to the standards in English Language Arts (National Association for Media Literacy Education, 2014) as part of a national curriculum in PK-12 classrooms. The teaching of media literacy competencies correlates especially well with those in the English Language Arts standards, since the instruction addresses an understanding of any message found in the media, be those based on visual, audio, print, or interactive texts.

NAMLE has identified five connection points with the CCSS that can be tied directly to media literacy instruction through proper assessment in project-based environments (National Association for Media Literacy Education, 2014). These connections include (a) the relationship between authors and audiences, (b) an expansion of the concept of literacy, (c) conducting research with current events, news, and information, (d) empowering students to be critical thinkers through media production and analysis, and (e) understanding differing points of view through ethical reflection (National Association for Media Literacy Education, 2014). Specific examples include the emphasis that, "the 
need to conduct research and to produce and consume media is embedded into every aspect of today's curriculum" (Common Core State Standards Initiative, 2016, p. 4), or that students should, "actively seek to understand other perspectives and cultures through reading and listening ... to communicate effectively with people of varied backgrounds" (Common Core State Standards Initiative, 2016, p. 7). These descriptions from the CCSS tie directly to key principles of media literacy, such as the view that it can be integrated across all subjects as a common base for understanding (Jolls et al., 2016), as well as its promotion of social justice through the representation and acceptance of diverse voices. The integration of media literacy competencies into the CCSS is a robust subject worthy of its own inquiry and is beyond the scope of this study. However, it is worth noting the policy reinforcement present in regard to media literacy instruction in PK-12 settings that is lacking in higher education.

Higher education settings. Media literacy as a subject in U.S. institutions of higher education is much less prevalent than in elementary or secondary education and can be seen as being nearly nonexistent in any kind of standardized fashion. Research in the field is also limited, with a few surveys administered in the mid-2000s still being the only organized attempt at understanding the breadth of media literacy instruction in university settings (Mihailidis, 2008). Media literacy is still unknown to most faculty. Those that are aware understand the need, however few are qualified to teach the topic (Schwarz et al., 2014). Similarly, most university students are also unaware of media literacy's definition or existence (Schwarz et al., 2014), with those not enrolled in mass communication or media studies majors unlikely to experience any type of media literacy instruction during their university career (Duran et al., 2008). Conversely, in a recent 
survey of current university students, a majority of respondents said that the inclusion of digital and media literacy elements within various curriculum positively enhanced the instruction (VideoBlocks EDU, 2015). The overall view of media literacy in higher education is one of duplicity; as new media and technology initiatives appear at ever-increasing rates, the ignorance regarding the subject persists.

Mihailidis (2008) identified inconsistent definitions, a focus of media literacy scholarship on PK-12 teaching, and the lack of adoption in the U.S. when compared to most other countries as being the main reasons why media literacy curriculum is difficult to pinpoint in higher education. Because of the inequality of adoption when compared to PK-12 settings, identifying any type of standardized learning outcomes in the university is difficult (Mihailidis, 2008). Assessment of accepted media literacy instruction outcomes has also largely been overlooked in higher education (Schilder et al., 2016). Further, due to its inherent nature, higher education has a scarcity of national standards similar to those enacted by the National Communication Association or the current Common Core standards. University faculty also tend to create their own curriculum, barring any administrative regulation, which would then be particular to only a single institution.

The limited research into the prevalence of media literacy at institutions of higher education suggests its inclusion is infrequent. Stuhlman and Silverblatt (2007) conducted a survey in 2002 , with a follow-up survey in 2007, that found only 158 colleges offering media literacy courses or programs. In 2004, a study conducted by a team of researchers at the University of Maryland surveyed 48 journalism and mass media programs in the U.S., inquiring about how faculty viewed media literacy, where it existed within their 
curriculum, and how they viewed media literacy as an instructional component (Mihailidis, 2008). The survey revealed acute pessimism towards media literacy from the faculty, with respondents singling out the irrelevance of the topic in higher education as well as general confusion about a basic definition (Mihailidis, 2008). A more recent study conducted by Schmidt (2012) found that faculty from varying disciplines identified the importance of students developing media literacy competencies, however most were unwilling to personally undertake this instruction due to systematic or personal factors. Yet these limited findings are problematic since media literacy competencies are not only taught in standalone courses and are often taught through an interdisciplinary method across the curriculum (Schmidt, 2013). Media literacy instruction at the post-secondary level suffers from inadequate data and misunderstandings of the specific learning outcomes that university students should be achieving (Mihailidis, 2008).

The inclusion of media literacy in university curriculum offers to teach students abilities such as critical thinking, an understanding of ethics and identity, connecting threads between media and civil society, and the ideals of engaged democracy (Mihailidis, 2008; Schwarz et al., 2014). Higher education aims to create independent learners who continue to pursue knowledge in a process of ongoing, lifelong learning. Gaining media literacy competencies while in college will give students the ability to utilize their skills as they continue to seek relevant information as an engaged citizen after graduation (Mihailidis, 2008).

The most straightforward discipline where media literacy would be taught is within mass communication and media studies programs. However, media literacy is 
taught in multiple settings in higher education. The most prevalent include (a) an integrated approach, which incorporates media literacy instruction into other disciplines, (b) as a general education requirement, (c) as a theoretical prerequisite in mass communication or media studies programs, (d) as a component of teacher education programs, or (e) as a discipline within a contemporary area of study such as advertising, business, or political science (Silverblatt, 2014). The next sections further investigate these five settings and the various disciplines within each.

Integrated approach. The integrated approach to teaching media literacy at the post-secondary level incorporates media literacy components into other established subjects such as language arts, social studies, and the health sciences (Silverblatt, 2014). This approach is the most similar to how media literacy instruction is included in elementary and secondary education settings. Though this method is not the most prevalent in higher education, integrating media literacy into other courses such as introductory communication courses has been successful (Silverblatt, 2014). This approach can be especially appealing to universities with already crowded curriculum requirements.

Proponents of this integrated approach often refer to foreign language instruction as a successful example (Silverblatt, 2014); students who immerse themselves in a language by visiting foreign countries are more successful compared to students who only speak the language for a few hours each week in an isolated classroom. By integrating media literacy instruction into other disciplines, the argument can be made that students will then integrate their newly formed competencies into other aspects of their life both in and out of the classroom. Since media literacy emphasizes critical thinking, it also can be understood to enhance those other subjects with which it is integrated (Silverblatt, 2014). 
There are, however, notable criticisms with taking this approach. When media literacy is not being taught as the primary subject, it is possible for faculty to overlook key principles necessary for successful pedagogical implementation (Silverblatt, 2014). Additionally, as was discussed in the previous section, most faculty do not feel they are prepared to teach the subject (Schwarz et al., 2014). For the integrated approach to be truly successful, faculty should have a background in communication, journalism, or media studies (Mihailidis, 2008); it would be difficult to find faculty with the required background teaching in a discipline outside of one of these areas.

General education requirements. Most universities do not offer standalone courses in media literacy; the few that do typically offer these classes as requirements for their general education programs (Christ, 2004; Silverblatt, 2014). Media literacy as a component of general education could take the form of a single course, or a set of courses that fulfill a critical thinking component, for example (Silverblatt, 2014). Due to a lack of empirical data, little is known regarding the specifics of these courses, such as the theoretical frameworks utilized or assessed learning outcomes. In the context of being a general education course that could potentially be taken by students from any discipline, it would be expected that instruction would be through a media lens, with a focus on critical media analysis (Mihailidis, 2008).

Theoretical prerequisite courses. As stated previously, the most obvious discipline in which one would expect to find standalone media literacy courses would be within mass communication, journalism, and media studies programs (Mihailidis, 2008). An example of a theoretical prerequisite course would be an introductory mass communication course, with a pedagogical focus on media literacy or including key components 
therein, for students majoring in media studies or a related field (Silverblatt, 2014). Like general education courses, these prerequisite courses would be taught through a media studies viewpoint, with a focus on critical analysis. These courses tend to include instructional elements focusing on communication processes, media regulation and ownership, the impact of media on society, and the history of different forms of media (Silverblatt, 2014).

Media literacy courses should be expected to exist as a component of all media studies and mass communication programs. However, if these programs are the only places where media literacy courses can be found, students majoring in other disciplines will be missing out on this learning. As Mihailidis (2008) states, "in the 21 st century media landscape, it is important for all university students to graduate with a basic understanding of the ways in which mediated information influences individuals, societies, and democracy." This is an imperative disparity that must be addressed in post-secondary institutions.

Teacher education. The majority of teachers at all grade levels are not trained in media studies and do not feel prepared to teach media literacy concepts to their students (Schilder et al., 2016; Schwarz et al., 2014). Pre-service teacher education programs in the U.S. often do not include media literacy concepts, and the field still has little support from established faculty or administrators (Kellner \& Share, 2005). Although teacher education programs are increasingly adding technology requirements to their curricula, various factors have hindered new teachers' ability to apply these skills in the classroom (Kleiner et al., 2007). While media literacy has been advancing in post-secondary teacher programs, this support usually entails a single course or an integrated approach, 
with the inclusion usually dependent on particular university faculty who have a personal interest in the subject (Kellner \& Share, 2005).

One of the most significant challenges facing media literacy is its recognition as a legitimate subject; a domino-effect will likely occur as more teacher education programs include the topic as part of their coursework, thus enabling more future teachers to pass on that knowledge to their students. Hobbs (2010) identified teacher education as being one of the key set of recommendations to further support the development of media literacy competencies for students. The three recommendations regarding teacher education include (a) support for interdisciplinary connections in post-secondary education that would allow for the integrations of media literacy into teacher education programs, (b) the creation of district-level policies to support media literacy in PK-12 settings through media and community partnerships, and (c) partnering with media companies to allow for current events to be better integrated into education programs with an aim to promote civic engagement (Hobbs, 2010).

Complementary areas of study. Similar to the integrated approach, media literacy as a subject can complement disparate fields of study such as health sciences, political science, business, and advertising (Silverblatt, 2014). Media literacy can either be integrated into these subjects in prerequisite courses, or as standalone courses taught through the lens of the discipline itself. One example could be advertising students critically analyzing media texts as reflections of societal values or cultural attitudes; an understanding of these messages could later inform the development of advertising campaigns (Silverblatt, 2014). Political science students could apply their media literacy 
competencies to analyze political campaigns and create political advertisements (Silverblatt, 2014). For students studying all of these disciplines, media literacy can offer an understanding of the ethical implications of their future professional work in real-world situations.

\section{Media Literacy Educational Frameworks}

It must first be established that today's students are engaged with, and have some kind of familiarity with, media literacy before taking part in any academic instruction in the classroom (Buckingham, 2007; Mihailidis et al., 2014a). While students have varying experience with digital technologies and media, this informal knowledge does not necessarily translate to competency or expertise in media literacy abilities (Mihailidis et al., 2014a). Since this study is focused on future university policy development regarding media literacy education, this scholarly review only focuses on formal media literacy instruction taking place in educational settings. The frameworks which are discussed in this section are all considered scholarly instruction based on the funding, support, and resources that are required for teaching media literacy in the classroom.

Media literacy is a critical thinking competency that comprises access, analysis, evaluation, and communication of media messages (Aufderheide, 1993; European Union, 2007; Hobbs, 2010). Media literacy education offers a viewpoint for understanding and negotiating these messages through active inquiry, self-expression, and critical thinking (Morrell et al., 2013; National Association of Media Literacy Education, 2007; Potter, 2004; Thoman \& Jolls, 2003). Acquisition of this literacy requires the competence to understand and describe different forms of communication, which in turn leads to the ability 
to utilize and interpret media through an expansive analytical understanding (Buckingham, 2003).

There are multiple frameworks in the academe currently being used for media literacy instruction, with each falling under the umbrella of either protectionism or empowerment. Protectionist frameworks guide students on how to overcome possible harmful influences from the media, while empowerment frames are based on students developing thinking skills regarding media that allow for them to fully participate in society (RobbGrieco, 2014). Though the debate between these two paradigms has generally been agreed upon, with most scholars and policymakers emphasizing empowerment over protectionism (Lunt \& Livingstone, 2012), it is still important to view the development of different frameworks over time to see how certain frames informed one another. This section creates a foundation for media literacy pedagogy based on some of the most oftcited conceptual frameworks, with an aim of developing a comprehensive pedagogical model for higher education at the conclusion of the scholarly review. Again, it must be emphasized that while students live in a media-saturated environment, the ability to learn about the media and understand what makes messages valid, factual, and valuable does not occur by coincidence, rather through formal media literacy instruction (Potter, 2004; Thoman \& Jolls, 2003).

Protectionist paradigm. Media literacy education was originally based upon a protectionist paradigm. This developed from a goal of protecting youth by providing cognitive defenses against the media to protect them from potential harm (HeeYoung Kim et al., 2016). Protectionists view media as being driven by politics and profits, without consideration of the public interest (RobbGrieco \& Hobbs, 2013). Protectionist 
frameworks are chiefly concerned with safeguarding vulnerable members of the public such as adolescents, the elderly, those with disabilities, as well as other historically disadvantaged groups such as minorities or those in the LGBTQ community (RobbGrieco \& Hobbs, 2013). A major limitation of this approach is that protectionism presents teachers with the greatest influence possible over students when interpreting and deconstructing media messages; this has been shown to lead to students becoming cynical towards media since they are seen as being unsuspecting targets of propaganda (HeeYoung Kim et al., 2016). Ultimately, the protectionist approach is seldom utilized currently for media literacy instruction in the U.S., but it does serve as a type of gateway for professionals in education and health care to ensure children are safeguarded against possible negative effects from media distortions (HeeYoung Kim et al., 2016; Lunt \& Livingstone, 2012).

Though most current media literacy frameworks are oriented towards empowerment over protection (Lunt \& Livingstone, 2012), it is still important to view how protectionist frameworks helped to establish the definitive definition for media literacy created at the 1992 Aspen Institute conference, as well as how it continued to develop in the years after the conference. A knowledge of the foundations of protectionist paradigms is essential for understanding current empowerment frameworks that have grown from both an intention of empowering students with critical thinking abilities, and the major shifts in our culture over the past few decades due to the growth of participatory networks and digital communication technologies. It should also be noted that the following protectionist frameworks did not develop sequentially, rather independent of one another at different time periods, as is explained further. 
Four core competencies. The most widely used definition of media literacy comes from the Aspen Institute conference proceedings over twenty years ago, which state that media literacy is the ability to access, analyze, evaluate, and create messages in a variety of forms (Aufderheide, 1993). These four components of access, analysis, evaluation, and content creation compose a basic, skills-based framework for media literacy education. The pedagogical basis of this model is one of learning through hands-on participation in a process-driven, group environment (Aufderheide, 1993). This framework is arranged in a nonlinear fashion, with any one component leading to a deeper understanding of the additional components in practice (Livingstone, 2004). While this definition is concise and contains the essential components of media literacy, it is limited to the extent that it is not sufficient in our current climate of diversification and convergence of media (Livingstone, 2002; Livingstone, 2004). However, it should be viewed as the definitive definition upon which all other media literacy educational frameworks are based.

Buckingham (2003) expanded on this basic framework by adopting a student-centered approach that is positioned around past experiences and pre-existing knowledge of the media. This modified framework somewhat de-emphasizes the protectionist stance of the Aspen Institute's original definition, and instead encourages students to make their own informed decisions regarding media interactions (Buckingham, 2003). The strengthening of Aufderheide's (1993) original definition by Buckingham (2003) can be viewed as an important shift in media literacy educational frameworks from protectionist intentions to empowerment objectives.

The four components of Aufderheide's (1993) definition have evolved here into four elements: representation, language, production, and audience (Buckingham, 2007). 
Buckingham (2007) defines representation as the media interpreting the world through selective ideologies and values. Students who are media literate are also expected to understand how the language employed by the media actually operates, instead of simply being able to use the same language (Buckingham, 2007). This ability is tied directly to Aufderheide's (1993) literacy component of access; in order to access a form of media, one must understand the language through which it is structured.

Production can also be tied to teaching media literacy through a protectionist lens (Mihailidis, 2009), since it focuses on the safety aspect of understanding who is communicating to whom (Buckingham, 2003; 2007). Being able to create content and communicate through media is an important aspect to this as well, however, understanding commercial and noncommercial influences is key to this ability (Buckingham, 2007). Audience, the final component of this framework, involves being aware of one's position as an audience member for particular forms of media, ensuring conscientiousness of how audiences are targeted and how those audiences then respond (Buckingham, 2007).

As stated earlier, Buckingham's (2003) work is a key arbiter of change towards an empowerment lens in media literacy education. While this framework is not wholly original and is rather a modification of Aufderheide's (1993) original definition, it was one of the earliest frameworks that explicitly focused on students themselves. Buckingham (2003) states:

Media education is now no longer so automatically opposed to students' experiences of the media. It does not begin from the view that the media are necessarily 
and inevitably harmful ... on the contrary, it adopts a more student-centered perspective, which begins from young people's existing knowledge and experience of media, rather than from the instructional imperatives of the teacher. (p. 13) This focal shift away from protecting youth against the negative effects of media towards empowering them through the use of their own cognitive abilities signals both a change in the paradigms of media literacy education and the creation of an instructional foundation based on viewing media literacy as a critical pedagogy. As will be discussed, empowerment through a critical pedagogy defines today's media literacy education frameworks. Before turning to current empowerment-based frameworks, however, an examination of two additional protectionist frames that developed independently of the Aspen Institute's definition will assist in understanding how the current frameworks originated.

Ideological analysis. Ideological analysis is a framework for better understanding the ways in which the media reflect, reinforce, and shape ideological systems (Silverblatt et al., 2009). The framework states that ideology, or the system of beliefs that determine how a culture thinks and behaves, is intrinsic to all aspects of media production, distribution, exchange, and consumption (Silverblatt, 2014). In his groundbreaking treatise on media education, Masterman (1985) acknowledged that facilitated discussion based on the questioning of ideological frames, in combination with teaching critical thinking skills, is a preferred method of media literacy instruction.

This media literacy framework is grounded in cultural studies, looking at media texts as a way to reveal how a society or culture operates. This cultural approach is based on four principles: (a) the inequitable distribution of power in society, (b) dominant 
forces inherent to our social system, (c) that similar ideologies are constantly repeated through a variety of media texts, and (d) that audiences absorb this additive cultural information and create new material from it, thus reinforcing dominant ideologies (Silverblatt et al., 2009). This foundational base of cultural studies allows educators who are utilizing the framework to focus on what media messages reveal about society, rather than on any aesthetic aspects of the message or effects it has on individuals.

There are six objectives included when conducting an ideological analysis of any media text. These objectives include (a) examining media messages as a way to identify the message's prevailing ideology, (b) enabling audiences to become more perceptive to ideology of content, (c) enabling the audience to understand the impact media has to reflect, reinforce, and shape a culture's ideology, (d) broadening the exposure of societal subcultures to others, (e) determining when ideological shifts take place within a culture, and (f) encouraging ideological withdrawal through the development of skepticism (Silverblatt et al., 2009). This approach can provide insight into the behaviors, values, and preoccupations of an audience or culture (Silverblatt, 2014). This framework is significant because it moves beyond simple descriptions of media texts to critical discussions about the values implicit in media texts, and whose interests are best served. Another key aspect to this approach is the concept of media not simply reflecting or reinforcing culture, but rather shaping thought by promoting the dominant ideology through cultural hegemony, which is the ability of dominant groups to maintain political and economic control over subordinates (Silverblatt, 2014).

Different lines of inquiry of ideological analysis include media ownership patterns, organizational analysis, worldview, historical contexts, point of view, rhetorical 
techniques, narrative analysis, genre analysis, and production elements (Silverblatt et al., 2009). Examples of using these lines of inquiry when examining a media text might include an understanding of how specific types of media ownership influence message construction, how the internal structures of media organizations impact content, or considering the point of view of a media message and understanding how the dominant ideology best serves the interest of that viewpoint (Silverblatt et al., 2009). Narrative analysis in this context can help audience members to discover the underlying assumptions of a message and separate themselves from any kind of unquestioned acceptance of it, while genre analysis allows for an understanding of any shared ideological orientations inherent in a particular genre of messages (Silverblatt et al., 2009). While these lines of inquiry are important and should be understood by any media literate person, this framework is limited in that it has the potential to develop an understanding of the construction of media messages, but it in no way offers the tools necessary for students to be literate citizens who are able to express themselves by questioning or changing these dominant ideologies.

Similar to Buckingham's (2003) modification of the Aspen Institute definition of media literacy, this framework can be seen as another significant shift away from protectionism towards empowerment. Through encouraging students to consider their ideological assumptions, they can learn to question what is considered the status quo (Funk et al., 2016). However, this framework also places much emphasis on organizational analysis, media ownership patterns, and rhetorical techniques for safeguarding oneself against manipulation, and should still be viewed as belonging to the protectionist paradigm. This framework suggests that media have emerged as one of the predominant methods through 
which ideology is introduced and reinforced, and that media can be understood as a product bequeathed to subordinates from the prevailing system. Effectively, this framework can be defined as one based on a protectionist approach that also includes some elements of critical pedagogy.

Empowerment paradigm. Empowerment frameworks are based on Dewey's (1938) emphasis on education being fundamental to civil societies. As was discussed in the section on the history of media literacy education, the empowerment approach began appearing in the U.S. in the 1960s and 1970s, during an era of significant educational reform (RobbGrieco \& Hobbs, 2013). The works of Paolo Friere (2010) were also significant to the development of the empowerment paradigm, which underscored the importance of critical analysis of the media and society in order for students to become truly engaged citizens.

Empowerment frameworks give students the ability to contribute their respective voices to society through media analysis and criticism, artistic expression, and production. Unlike protectionist frameworks, the empowerment paradigm approaches issues such as media control and influence in distinctive ways. Empowerment views media power imbalances as a participation gap, and precarious uses of media as ethical dilemmas; all of these issues are seen not as obstacles that students must be guarded from, rather as challenges which can be overcome through the attainment of media literacy competencies (RobbGrieco \& Hobbs, 2013).

Though there are multiple empowerment frameworks in the academy, the most common themes that appear among them include elements of critical thinking, critical reflection, inquiry-based instruction, group and project-based learning, and creative media 
production. Empowerment frameworks have become the predominant pedagogical approach to teaching media literacy over the past two decades, in conjunction with new media technologies and shifts in how individuals and societies communicate. While these frameworks have often developed independently of one another yet also simultaneously, they are presented here in a specific order. These frameworks should be viewed as building upon one another, from a focus on cognitive abilities to inquiry-based learning, then incorporating new media literacies and media production, which then culminate in the critical media literacy framework which incorporates elements of all of these approaches.

Cognitive model of media literacy. Potter (2004) continued Buckingham's (2003) transition from a protectionist to an empowerment lens with the cognitive model of media literacy, which is based on cognitive science and psychology. Potter (2004) defines media literacy as being able to, "empower individuals to shift control from the media to themselves" (p. 68). He also states that media literacy supports citizens' ability to adapt to a constantly shifting reality through an understanding of media messages and the ability to construct messages to convey that understanding to others (Potter, 2013). A cornerstone of this cognitive model is the concept of media literacy knowledge being perpetual, with individuals placed somewhere along a continuum based on their knowledge and media literacy competencies (Potter, 2013).

The cognitive model of media literacy is based on four important factors, the foundation level being knowledge structures (Potter, 2004). Knowledge structures are organized sets of information in one's memory, built up over time with attention and close precision (Potter, 2013). Information is the fundamental element of knowledge structures; we organize information into patterns which later help us recall knowledge. In 
terms of media literacy, this framework includes five knowledge structures: (a) media effects, (b) media content, (c) media industries, (d) the real world, and (e) the self. Potter (2004) suggests that if students acquire an understanding of these five knowledge structures, they will be better able to seek out useful media messages to serve their personal goals. Individuals who possess more experiences in the real world are better able to analyze and acknowledge media content (Potter, 2013). These knowledge structures provide a context through which media messages can be understood; the more knowledge structures one has, the better able one is to critically understand these messages.

The five knowledge structures then inform students' personal locus, the second factor of this model. This is the point where personal goals determine future informationprocessing tasks through the filtering of media messages (Potter, 2004). This stage is critical in the media literacy development process; the more actively engaged a student is with his or her locus, the more aware they will be about their decision-making processes (Potter, 2004). However, when someone has a weak locus, they tend to allow the media to operate with a great deal of control over their exposure to media texts and processing of information (Potter, 2013). Seemingly, the more knowledge one has about their personal locus, the better disciplined they are in regard to media influence. Since media literacy is a process rather than a product, gaining this competency means that a person engages with their locus more often over time (Potter, 2013).

The third factor of the cognitive model is a student's competencies and skills, which are the tools that are used in upcoming information-processing tasks (Potter, 2004). In order to construct knowledge structures, one must rely on their own skills. 
Potter (2013) identifies seven skills necessary for media literacy: (a) analysis, (b) evaluation, (c) grouping, (d) induction, (e) deduction, (f) synthesis, and (g) abstracting. Analysis is the deconstruction of media messages into the essential elements, while evaluation involves making value judgements based on any message element (Potter, 2013). Grouping searches for similar elements in a message and then grouping those elements together (Potter, 2013). Induction ascertains patterns among a small number of elements into larger sets, while deduction achieves the opposite in utilizing general principles to understand particular elements (Potter, 2013). Finally, skills in synthesis means one can assemble message elements into new structures, while abstracting is the process of creating an accurate description of the substance of a media message (Potter, 2013). Potter (2004) warns, however, that, "having competencies does not make one media literate, but lacking these competencies prevents one from being media literate, because this prevents a person from accessing particular kinds of information." (p. 70)

The fourth factor culminates in the flow of information-processing tasks (Potter, 2004). These tasks include filtering, meaning matching, and meaning construction, and are in an ordered sequence of actions (Potter, 2004). When we receive a media message, it must first be either ignored or filtered into our consciousness. If a message is selected to be filtered in, the layers of meanings within that message must then be recognized. Being media literate means that people do not unconsciously accept media messages, but rather through the development of skepticism one actively constructs meaning after exposure to these messages (Potter, 2004). This model is founded on a continual process of developing these skills, which leads to the continued development of further knowledge structures, ultimately making one more media literate. 
According to this model, as an individual progresses along the developmental continuum, they advance to more competent levels of media literacy skills (see Figure 2) (Potter, 2013). The lowest levels, such as acquiring language and narrative, happen in the early years of life. The development of skepticism and the motivation to continually learn occurs in childhood and young adulthood; many people do not progress past the stage of intensive development at any point during their lifetime (Potter, 2013). Once the intensive development stage is reached, people often incorrectly consider themselves to be media literate since they are able to access the messages they are seeking out and can achieve a meaning from those messages. 


\section{Figure 2}

Development of Media Literacy

\section{$\underline{\text { Development of Media Literacy }}$}

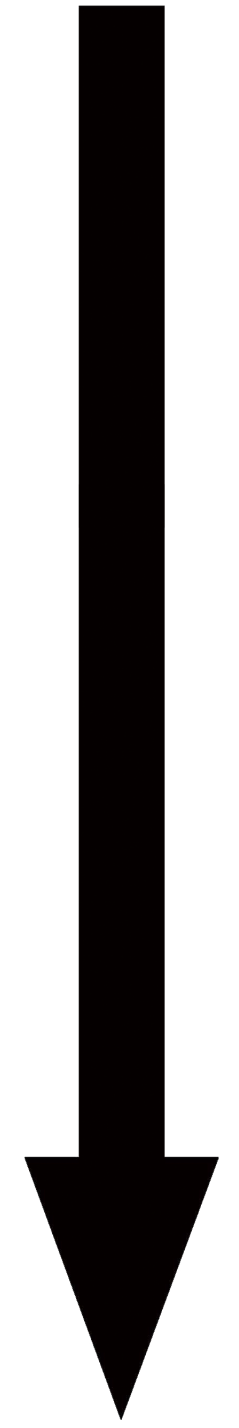

Acquiring

fundamentals

Language

acquisition

Narrative

acquisition

Developing

skepticism

Intensive

development

Experiential

exploring

Critical

appreciation

Social

responsibility
Concepts of time, shape, form, facial expressions, physical beings

Recognizing and reproducing speech sounds

Understand differences in real and make-believe

Discounting claims, understanding differences between likes and dislikes

Motivation to seek out information, quick facility in information processing

Seeking different narratives, searching for new aesthetic reactions

Evaluating messages, broad understanding of message contexts

Recognition of individual decisions affecting society

Note. This figure shows the different stages individuals move along as they develop media literacy competencies as suggested by Potter's (2004) cognitive model. Adapted from Media literacy (6 ${ }^{\text {th }}$ ed.), by W. J. Potter, 2013, Thousand Oaks, CA: SAGE. 
Only the final three stages of the development process can be seen as advanced and to contain the critical thinking skills inherent in media literacy. Experiential exploring is the realization that an individual's media exposure might in fact be narrow and works to remedy the situation by seeking out a wider range of media texts and sources (Potter, 2013). At the critical appreciation stage, people have the ability to seek out superior messages based on cognitive, emotional, moral, and aesthetic grounds (Potter, 2013). Finally, social responsibility symbolizes a critical understanding of all types of media messages with an external recognition of which messages are best for society (Potter, 2013). Moving among these stages of development requires a mindful intention to grow one's media literacy competencies. The benefits of developing a higher degree of media literacy include an understanding of a wider variety of messages and better self-control over individualized thought patterns in regard to the possible influences of media (Potter, 2004).

As stated earlier, Potter's (2004) model is an important shift from the earlier goals of developing media literacy skills in order to protect youth, to one of empowering them to develop active thinking skills and be more conscious of their media environment. The key is to encourage students to engage with media often in order to gain conscious processing skills (Potter, 2004). However, there are shortcomings in the aforementioned model. This framework was constructed more than a decade ago, and in that time the media landscape has changed significantly to now emphasizing new media platforms where students are creating their own content in what can best be defined as a participa- 
tory culture (Jenkins et al., 2009). It should also be noted that while media literacy requires reflective thinking, a continuous, high level of cognitive engagement is not possible in our current media-saturated reality (Austin \& Silverblatt, 2014).

Buckingham (2005) has emphasized that media literacy should not solely be viewed as a cognitive ability, and should instead also include cultural acknowledgment, emotional responses, and some form of gratification. Potter's (2004) framework focuses only on cognitive abilities and is missing production aspects seen as critical to a current understanding of media literacy. In order to foster media literacy that encourages students to become full participants in today's participatory culture, additional attention must be given to contemporary media literacies that include social skills based on collaboration, as well as a shift in the teacher-student relationship which encourages collaboration through the facilitation of highly structured questioning. These new competencies are built upon the foundation of critical thinking skills originating from earlier frameworks such as Potter's (2004) cognitive model.

Inquiry-based pedagogy. Inquiry-based instruction in media literacy is established on the use of relevant questions to evaluate and analyze media messages. Specifically, this pedagogical approach is undertaken by teachers, working collaboratively with their students, to link students' personal encounters with the media in order to enhance their overall learning experience (Silverblatt, 2014). Teaching media literacy through this framework has shown that as students are exposed to more media within this environment, they are better able to engage in analytical discussions about media texts and also become more accepting of differing opinions (HeeYoung Kim et al., 2016). This framework is unique, when compared to the other empowerment frameworks, in that it 
does not necessarily introduce media literacy as a new subject to teach, but rather views media literacy as a new lens through which to teach and learn (Silverblatt, 2014).

Key to this framework is the view that the traditional hierarchical nature of the student-teacher relationship will often inhibit learning, as suggested by Friere (2010). The role of instructor can be viewed as shifting from an authoritarian figure to a discussion facilitator (Silverblatt, 2014). Masterman (1985) noted that media literacy instruction should avoid attempting to have students simply reproduce the ideas or insights provided by the teacher. In order to engage students and develop their inquiry skills, teachers should instead model the methods to analyze and evaluate media messages by utilizing highly structured questioning, which then leads to students using their own understanding of media texts to engage in critical thinking (HeeYoung Kim et al., 2016). Questioning is the most important aspect of an inquiry-based pedagogy, with critical thinking arising from an awareness of sets of interrelated questions, abilities in asking and answering questions, and the active desire to utilize questioning (Browne \& Keeley, 2001).

This framework should be viewed as a democratic pedagogy as it challenges typical hierarchical classroom structures. Because of the egalitarian arrangement, it is inevitable that some course content will arise that is unfamiliar to the instructor. An inquiry approach works well in this situation, however, because it establishes listening as the most important skill for media literacy instructors to possess (Silverblatt, 2014). With the teacher no longer managing a classroom, but now moderating it instead, engagement among students and teacher can grow, leading to further critical understanding and discussion (Silverblatt, 2014). In this collaborative setting, teaching students how to ask 
specific types of questions allows for a deeper understanding of media texts (National Association of Media Literacy Education, 2007). Teachers successfully utilizing an inquiry-based approach are not expected to be truly objective, rather they should understand classroom dynamics and the appropriate time to voice their personal opinion so as not to curtail student inquiry (Scheibe \& Rogow, 2012).

The National Association for Media Literacy Education (NAMLE) produced a guide for teachers undertaking inquiry-based instruction, titled Key Questions To Ask When Analyzing Media Messages (see Figure 3) (National Association of Media Literacy Education, 2007). The guide provides different types of questions for students to ask that consider how authors, meanings, and representations might influence media messages. 


\section{Figure 3}

Key Questions to Ask When Analyzing Media Messages

\begin{tabular}{|c|c|c|}
\hline & & SAMPLE QUESTIONS \\
\hline \multirow{5}{*}{$\begin{array}{l}\text { Audience and } \\
\text { Authorship }\end{array}$} & AUTHORSHIP & Who made this message? \\
\hline & PURPOSE & $\begin{array}{l}\text { Why was this made? } \\
\text { Who is the target audience (and how do } \\
\text { you know)? }\end{array}$ \\
\hline & ECONOMICS & Who paid for this? \\
\hline & IMPACT & $\begin{array}{l}\text { Who might benefit from this message? } \\
\text { Who might be harmed by it? } \\
\text { Why might this message matter to me? }\end{array}$ \\
\hline & RESPONSE & $\begin{array}{l}\text { What kinds of actions might I take in } \\
\text { response to this message? }\end{array}$ \\
\hline \multirow{3}{*}{$\begin{array}{l}\text { Messages and } \\
\text { Meanings }\end{array}$} & CONTENT & $\begin{array}{l}\text { What is this about (and what makes you } \\
\text { think that)? } \\
\text { What ideas, values, information, and/or } \\
\text { points of view are overt? Implied? } \\
\text { What is left out of this message that } \\
\text { might be important to know? }\end{array}$ \\
\hline & TECHNIQUES & $\begin{array}{l}\text { What techniques are used? } \\
\text { Why were those techniques used? } \\
\text { How do they communicate the message? }\end{array}$ \\
\hline & $\begin{array}{l}\text { INTERPRETATION } \\
\text { S }\end{array}$ & $\begin{array}{l}\text { How might different people understand } \\
\text { this message differently? } \\
\text { What is my interpretation of this and } \\
\text { what do I learn about myself from my } \\
\text { reaction or interpretation? }\end{array}$ \\
\hline \multirow[b]{2}{*}{$\begin{array}{l}\text { Representations } \\
\text { and Reality }\end{array}$} & CONTEXT & $\begin{array}{l}\text { When was this made? } \\
\text { Where or how was it shared with the } \\
\text { public? }\end{array}$ \\
\hline & CREDIBILITY & $\begin{array}{l}\text { Is this fact, opinion, or something else? } \\
\text { How credible is this (and what makes } \\
\text { you think that)? } \\
\text { What are the sources of the information, } \\
\text { ideas, or assertions? }\end{array}$ \\
\hline
\end{tabular}

Note. This figure lists suggested questions to ask students to explore how authors, meanings, and representations might influence media messages. Adapted from Core Principles Key Questions, In The Core Principles of Media Literacy Education, n.d., Retrieved July 23, 2016, from https://namle.net/publications/core-principles/. Copyright 2007 by The National Association for Media Literacy Education. 
Example questions investigate who created a message and for what benefit, the production techniques used, the credibility of messages, and the ideas or values behind a message (National Association of Media Literacy Education, 2007). Because the questions are based on broad categories and concepts rather than specific questions, instructors are able to utilize different questions in differing classroom situations; this also allows for the key questions to be successfully used at any grade level (HeeYoung Kim et al., 2016). Instead of guiding students to specific answers, the inquiry-based approach prepares students with the ability to independently analyze media texts inside and outside of the classroom (Silverblatt, 2014).

Media literacy lends itself well to inquiry-based instruction because it is a process-oriented pedagogy (Silverblatt, 2014). While the inquiry approach can be viewed as its own framework, it can also be integrated with the other empowerment frameworks considered in this study. An example of combining these frameworks in practice is to ask students to bring in their own examples of media messages to discuss in class. Handing control over to the students allows them to critically engage with new media texts they are already familiar with, and through inquiry teachers can help guide students to using their cognitive abilities to effectively analyze media texts.

New media literacies. New media literacies are founded upon the recent convergence of media platforms as defined by media scholar Henry Jenkins. Jenkins (2006) defines media convergence as the collaborative partnership among differing media industries that allows content to appear across multiple platforms. This collaboration has become commonplace over the past decade; an example is an audience member watching a 
television show while simultaneously interacting with fellow audience members on a social media platform through the utilization of a hashtag specific to that program. Media convergence has allowed for the creation of participatory cultures, which are defined as cultures with, "relatively low barriers to artistic expression and civic engagement, strong support for creating and sharing creations, and some type of informal mentorship whereby experienced participants pass along knowledge to novices" (Jenkins et al., 2009, p. xi). In a participatory culture, collaborators see their contributions as being significant while feeling a degree of social connection with other participants. Our current participatory culture is based on a constant connectedness through social media, mobile access, and other innovative digital technologies.

Dynamic communication technologies have created an interactive media environment where consumers become prosumers; a proficiency in being able to create and distribute original content has become a requirement for current students (Chen et al., 2011; Literat, 2014). This new media landscape offers, "opportunities for peer-to-peer learning, a changed attitude toward intellectual property, the diversification of cultural expression, the development of skills valued in the modern workplace, and a more empowered conception of citizenship" (Jenkins et al., 2009, p. xii). While some have argued that students acquire these competencies independently of formal education through mere access, three challenges warrant pedagogical and policy interventions: (a) a participation gap in the form of unequal access to the experiences, skills, and opportunities needed for all students to fully participate, (b) students facing an uphill battle in recognizing the ways in which media shape perceptions, and (c) shortcomings in an ethical understanding 
of public representation that comes with participation that could be addressed through proper education (Jenkins et al., 2009).

The necessary competencies for navigating this participatory culture were outlined by Jenkins et al. (2009) into eleven new media literacies. These skills include (a) play, (b) performance, (c) simulation, (d) appropriation, (e) multitasking, (f) distributed cognition, (g) collective intelligence, (h) judgement, (i) transmedia navigation, (j) networking, and (k) negotiation (see Figure 4) (Jenkins et al., 2009). 


\section{Figure 4}

New Media Literacies

\begin{tabular}{|c|c|}
\hline \multicolumn{2}{|c|}{ New Media Literacies } \\
\hline PLAY & Experiment with surroundings in order to solve problems \\
\hline PERFORMANCE & $\begin{array}{l}\text { Adopt alternative identities for the purpose of discovery and } \\
\text { improvisation }\end{array}$ \\
\hline SIMULATION & Interpret and construct models of real-world processes \\
\hline APPROPRIATION & Sample and remix media content in a meaningful way \\
\hline MULTITASKING & Shift focus onto details based on the environment \\
\hline DISTRIBUTED COGNITION & Meaningfully interact with tools that expand mental capacity \\
\hline $\begin{array}{l}\text { COLLECTIVE } \\
\text { INTELLIGENCE }\end{array}$ & Work with others to pool knowledge towards a common goal \\
\hline JUDGMENT & Evaluate the credibility and reliability of information sources \\
\hline TRANSMEDIA NAVIGATION & Follow the flow of stories across multiple media forms \\
\hline NETWORKING & $\begin{array}{l}\text { Being able to search for, synthesize, and disseminate } \\
\text { information }\end{array}$ \\
\hline NEGOTIATION & Respecting multiple perspectives from diverse communities \\
\hline
\end{tabular}

Note. This figure shows the different competencies required for successful navigation in participatory cultures. Adapted from Confronting the challenges of participatory culture: Media education for the 21st century, by H. Jenkins, R. Purushotma, M. Weigel, K. Clinton, \& A. J. Robinson, 2009, Cambridge, MA: MIT Press. 
The new media literacy framework diverges from previous frameworks with its emphasis on active participation through both media consumption and creation. These new media literacies are viewed by Jenkins et al. (2009) as being cultural and social competencies that move beyond technological access to critical thinking skills augmented through direct interaction with the participatory culture. Social skills are seen as paramount to the development of new media literacies gained through networking and collaboration (Jenkins et al., 2009).

The skill of play means having the willingness to experiment with one's surroundings as a way of problem solving, while performance is the ability to accept alternative circumstances in order to improvise and discover (Jenkins et al., 2009). Simulation is having the capacity to interpret and construct dynamic representations of real activities, while appropriation means being able to sample and remix existing media content (Jenkins et al., 2009). The multitasking skill is seen as the ability to scan one's environment and shift focus to important details; distributed cognition is being able to meaningfully interact with digital tools in order to expand one's awareness and mental capacity (Jenkins et al., 2009). Collective intelligence is a competency in being able to pool knowledge and compare differences with others with an aim of reaching a common goal, while judgment is the ability to evaluate the credibility and reliability of information (Jenkins et al., 2009). Having the capacity to navigate the flow of multiple stories across multiple media platforms is represented by transmedia navigation, with networking being the ability to search, synthesize, and promulgate information (Jenkins et al., 2009). Fi- 
nally, the negotiation skill is the having the ability to move among diverse groups, understanding and respecting varying perspectives, and being perceptive to alternative norms (Jenkins et al., 2009).

This framework focuses on the concept of participatory cultures in preference to discussions on specific interactive technologies. Rather than viewing new media literacies against technology in isolation, Jenkins et al. (Jenkins et al., 2009) argue instead for an ecological approach focusing on the interrelationships among different media platforms and communication technologies, the cultures that manifest around them, and the interactions supported by the tools. Media are viewed here as operating within specific cultural and institutional contexts, which inform their use (Jenkins et al., 2009). Through participation, students are able to acquire the necessary skills to ensure their future success. This framework is taking a skills-based approach to media literacy, viewed through the lens of participatory culture in order for students to develop the skills, knowledge, ethical understanding, and determination to become full participants in our current culture (Jenkins et al., 2009).

As stated previously in this study, a review of the existent literature shows that one of the most significant shortcomings of media literacy instruction in higher education settings is the lack of appropriate measurement tools to assess learning outcomes (Mihailidis, 2008). Scholars have long remonstrated over the need to create a national standard for media literacy assessment as well (Scharrer, 2002). Unlike many of the frameworks covered in this scholarly review, the new media literacies framework as presented by Jen- 
kins et al. (2009) underwent an independent study by Literat (2014); the study is significant because it is one of the few quantitative studies undertaken to assess new media literacy competencies.

The intent of the Literat (2014) study was to address the methodological and assessment disparities present in media literacy research through the development and validation of a comprehensive assessment tool that could measure actual learning outcomes against the new media literacies as proposed by Jenkins et al. (Jenkins et al., 2009). The study measured twelve new media literacies and viewed both consumption and production of media as an understanding of media literacy; a twelfth literacy of visualization, or the ability to comprehend and create visual representations of information (Literat, 2014), was added to the study from a previous version of the Jenkins et al. (Jenkins et al., 2009) framework. The study also explored the relationships between the new media literacy skills of the students with levels of civic engagement, digital participation, and media exposure (Literat, 2014).

Utilizing a factor analysis methodology, the study found that ten of the twelve new media literacy skills were significantly relevant to a comprehensive understanding of media literacy (Literat, 2014). Only the skills of simulation and collective intelligence were not found to have a significant relationship (Literat, 2014). A positive relationship was also found between higher levels of new media literacy skills and increased civic engagement, increased abilities in media creation, and increased levels of media exposure. An additional finding was that higher levels of new media literacy skills only corresponded to new forms of media such as social media, the Internet, and video games, and not traditional media such as television and print (Literat, 2014). This study validated the 
new media literacy framework as presented by Jenkins et al. (Jenkins et al., 2009), and presented important correlations between media literacy proficiency and increased civic engagement and media participation. It is also exposed limitations in that these new media literacies only correspond to more recent, interactive technologies and not to more traditional, passive media. It is, however, a significant step towards the creation of a standardized assessment tool for media literacy education.

The new media literacy framework is an essential component of a current understanding of media literacy pedagogy; it addresses issues of understanding how students interact with interactive digital media and confirms the assumption that higher levels of media literacy translate to increased levels of civic engagement and media participation. The development of this framework also introduced the concept of participatory culture, which is the most accurate definition of the current setting in which students are interacting with the media. The framework also builds on the collaborative approach present in inquiry-based pedagogy but is lacking in the thoroughness of understanding the cognitive abilities required of a media literate person. Media creation is an important aspect of this framework; however, the next framework in this scholarly review, that of critical media production, addresses media creation in expanded terms.

Media production approach. Media literacy education ultimately allows for the emergence of digital citizens who will use their media-savvy influence to foster responsible dialogue and action in our participatory culture. Regrettably, the education system in the U.S. has been reluctant to acknowledge the benefits of students engaging with different forms of participatory culture such as collaborative problem solving and expressing 
oneself through media production and sharing (Jenkins et al., 2009). The media production approach gives students the opportunity to learn media literacy concepts through hands-on experience (Silverblatt, 2014). Media production also promotes the development of more knowledgeable citizens in our society (Morrell et al., 2013).

The production approach to media literacy is not intended to produce media professionals, rather to strengthen all students' theoretical understanding of media texts through direct engagement with the production process (Silverblatt, 2014). A thorough understanding of media production components such as lighting, sound, subjectivity, positioning, and editing leads to a better understanding of the processes taking place in the background before a media message is introduced to the public. Similar to the media production approach, and worth noting, is the production analysis approach that focuses on the utilization of production elements as a method for developing a critical understanding of texts (Silverblatt, 2014). When combined with the act of production itself, an analysis of production techniques also helps with an appreciation of media aesthetics, the development of social skills through collaborative work, and an understanding of the production process (Silverblatt, 2014).

Guided by Friere's (2010) work on emancipation via pedagogy and Brookfield et al.'s (2012) research on critical thinking as a reflective thought process, the media production framework for media literacy is based on the idea that critical pedagogy fosters creativity and critical media production (Morrell et al., 2013). The frame views critical media education as a process that makes students aware of the role media has in shaping our society (Kellner \& Share, 2005; Morrell et al., 2013). This framework shares aspects 
of critical pedagogy with the upcoming framework of critical media literacy as transformative pedagogy; however, it differentiates itself from all other frameworks in this study with its emphasis on active, firsthand media production. While this critical framework stresses production, it also corresponds to the literature emphasizing media literacy education's ability to foster participatory democratic engagement through critical access, analysis, communication, and creation of media messages (Buckingham, 2003; European Union, 2007; Hobbs, 2010; Mihailidis, 2008).

The media production approach exemplifies how today's students share analytical thinking with their peers through social action and awareness (Morrell et al., 2013). In practice, Morrell et al.'s (2013) model for critical media production positions learning as active, authentic, participatory, and empowering (see Figure 5). For learning to be active, students must participate in the real-world creating things that have personal meaning, while authenticity is only achieved when tasks have purpose (Morrell et al., 2013). Participatory learning is viewed as only occurring when learning is happening within a collaborative community of both like and dissimilar peers (Morrell et al., 2013). Finally, for learning to be empowering, it, "must give students power to act differently upon the world, and they must be aware of how their learning in formal environments translates into power in academics, in the professional world, and in civic life" (Morrell et al., 2013, p. 16). The benefits of producing media in a formal learning environment include active, constructive creation and student empowerment through real-world problem-solving (Funk et al., 2016). 


\section{Figure 5}

Critical Media Production Model

\begin{tabular}{|c|c|}
\hline \multicolumn{2}{|l|}{ Learning must be... } \\
\hline ACTIVE & $\begin{array}{l}\text { Learners must be doing things that matter to them in the } \\
\text { real world }\end{array}$ \\
\hline AUTHENTIC & $\begin{array}{l}\text { Tasks must have meaning and purpose whenever } \\
\text { possible }\end{array}$ \\
\hline PARTICIPATORY & Learning happens within a diverse community \\
\hline EMPOWERING & Learning gives students the power to enact change \\
\hline
\end{tabular}

Note. This figure shows the components of Morrell et al.'s (2013) model for critical media production. Adapted from Critical media pedagogy: Teaching for achievement in city schools, by E. Morrell, R. Dueñas, V. Garcia, and J. Lopez, 2013, New York, NY: Teacher's College Press.

Studies have shown creative media production to account for significantly greater levels of media literacy when compared to exclusively consumption-based pedagogies (Phang \& Schaefer, 2009). Like project-based learning, taking part in the process of production is where most learning actually occurs (Funk et al., 2016). That is not to say that media literacy instruction should be solely focused on production, however, this shows the importance of including active, participatory activities such as creative production with other media literacy pedagogies. This emphasis on active participation ties directly to the new media literacy framework espoused by Jenkins et al. (2009). 
Brookfield (2012) states that the process for critical thinking takes place through four steps: (a) identifying assumptions that frame our thinking, (b) understanding the validity of these assumptions, (c) viewing our decisions and ideas from multiple perspectives, and (d) taking informed actions based on these steps. It can be argued that the media production approach strongly emphasizes the fourth step, while not entirely dismissing, yet noticeably de-emphasizing the other three steps. For students to gain critical media literacy proficiencies, a framework must be utilized that includes all four of these steps. The next and final framework in this study, that of critical media literacy as transformative pedagogy, considers these limitations.

Critical media literacy as transformative pedagogy. The teaching of media literacy in our multicultural society naturally brings to students' attention the inequities present in society based on class, race, and gender discrimination (Kellner \& Share, 2005). Cultural studies have exhibited the pervasiveness of media in contemporary society and the need for media literacy to address issues of injustice, multiculturalism, and social differences (Kellner \& Share, 2005). Critical media literacy instruction empowers students to critically interpret information in multiple formats, to question the status quo, and to become change agents (Funk et al., 2016).

Critical media literacy pedagogy is highly influenced by Freire's (2010) critical pedagogy framework, and it recognizes the political nature of education (Funk et al., 2016) through challenging the societal hierarchies present in media texts (HeeYoung Kim et al., 2016). A critical media pedagogy ties directly to the idea of what it means to be an active citizen in today's participatory culture. This frame displays an important method 
for strengthening civic engagement among students by reaffirming the notion of democracy through empowerment (Funk et al., 2016). As mentioned previously when discussing the settings of media literacy instruction in higher education, critical media literacy pedagogy is especially significant when utilized in teacher education programs; this framework helps pre-service teachers to mentor their students in critical inquiry regarding digital communication technologies and popular culture encountered outside the classroom setting (Funk et al., 2016).

Critical media literacy instruction takes place in a collaborative environment that empowers students and teachers to use their knowledge and skills to be active citizens who challenge societal injustices (Funk et al., 2016). For critical pedagogy to be successful, teachers must accept a bottom-up approach that allows for students to bring their own experiences, culture, and insight to the classroom (Kellner \& Share, 2005). This pedagogy prepares students to analyze media message conventions, criticize dominant ideologies and stereotypes, and interpret the meanings intrinsic to media texts (Kellner \& Share, 2005). In comparison with the other empowerment frameworks presented in this study, true critical media literacy pedagogy legitimizes student perspectives to the greatest degree through the understanding of media texts as products of social production and its emphasis on self-expression and social activism (Kellner \& Share, 2005). It is also a transformative pedagogy through the utilization of a theoretical foundation combined with practical teaching methodologies that empower students to critically scrutinize dominant ideologies (Funk et al., 2016).

Unlike the previous empowerment approaches, the critical media literacy framework is not a traditional pedagogy with well-established texts, assumptions, or teaching 
methods. Instead, it is a democratic pedagogy that involves teachers sharing classroom control with students as they work collaboratively to challenge authority (Kellner \& Share, 2005), similar to the inquiry-based approach. Funk et al. (2016), however, developed a set of six concepts and questions that can be used as a structural framework for this critical instruction. These six concepts were adapted from the Center for Media Literacy's five core concepts of media literacy that were presented earlier in this scholarly review (Thoman \& Jolls, 2003). These six concepts include (a) social constructivism, (b) language and semiotics, (c) audience and positionality, (d) politics of representation, (e) production and institutions, and (f) social justice (see Figure 6) (Funk et al., 2016). 


\section{Figure 6}

Critical Media Literacy Questions and Concepts

\begin{tabular}{|c|c|c|}
\hline \multicolumn{3}{|c|}{ Critical Media Literacy Questions and Concepts } \\
\hline $\begin{array}{l}\text { Social } \\
\text { Constructivism }\end{array}$ & $\begin{array}{l}\text { Which people made } \\
\text { the choices in } \\
\text { creation of this } \\
\text { message? }\end{array}$ & $\begin{array}{l}\text { Media messages are created by } \\
\text { individuals and/or groups making } \\
\text { choices within social contexts. }\end{array}$ \\
\hline $\begin{array}{l}\text { Languages / } \\
\text { Semiotics }\end{array}$ & $\begin{array}{l}\text { How was a message } \\
\text { constructed and } \\
\text { delivered? }\end{array}$ & $\begin{array}{l}\text { Each medium has its own particular } \\
\text { language/grammar and semantics. }\end{array}$ \\
\hline $\begin{array}{l}\text { Audience / } \\
\text { Positionality }\end{array}$ & $\begin{array}{l}\text { In what ways can } \\
\text { this message be } \\
\text { understood } \\
\text { differently? }\end{array}$ & $\begin{array}{l}\text { People understand media messages in } \\
\text { differing ways based on multiple } \\
\text { contextual factors. }\end{array}$ \\
\hline $\begin{array}{l}\text { Politics of } \\
\text { Representation }\end{array}$ & $\begin{array}{l}\text { What values, points } \\
\text { of view, or ideologies } \\
\text { are represented in } \\
\text { this message? }\end{array}$ & $\begin{array}{l}\text { Biases that support or challenge the } \\
\text { hierarchies of power and privilege are } \\
\text { always present in messages and the } \\
\text { mediums through which they are } \\
\text { delivered. }\end{array}$ \\
\hline $\begin{array}{l}\text { Production / } \\
\text { Institutions }\end{array}$ & $\begin{array}{l}\text { Why was this } \\
\text { message created } \\
\text { and/or shared? }\end{array}$ & $\begin{array}{l}\text { All messages have a purpose shaped by } \\
\text { their creators or the systems in which } \\
\text { they operate. }\end{array}$ \\
\hline Social Justice & $\begin{array}{l}\text { Whom does this } \\
\text { message advantage } \\
\text { or disadvantage? }\end{array}$ & $\begin{array}{l}\text { Messages perpetuate or challenge ideas } \\
\text { about individuals, groups, and/or issues. }\end{array}$ \\
\hline
\end{tabular}

Note. This figure shows the questions and explanations that guide students to a critical inquiry of any media message. Adapted from Critical media literacy as transformative pedagogy, by S. Funk, D. Kellner, and J. Share, 2016, in M. N. Yildiz and J. Keengwe (Eds.), Handbook of Research on Media Literacy in the Digital Age, (pp. 1-30), Hershey, PA: Information Science Reference.

Social constructivism examines how messages are constructed by individuals or groups, and the social contexts within which they are created (Funk et al., 2016). Semiotics acknowledges that each medium has its own language with semantics and grammar specific to that medium (Funk et al., 2016). Positionality in this framework looks at how 
different people understand media texts in similar or dissimilar ways, along with any contributing contextual factors (Funk et al., 2016). Politics of representation recognizes the biases, ideologies, and power inherent in different mediums and messages (Funk et al., 2016). Production views all messages as having a purpose shaped by their creators, and finally social justice, in this framework, views media culture as an ecosystem that perpetuates or challenges societal ideas (Funk et al., 2016).

It was noted in the previous framework section that the media production approach focuses principally on the fourth step of Brookfield's (2012) process of critical thinking, that of taking informed actions. The critical media literacy framework addresses all four steps; the identification of assumptions are addressed through positionality and semiotics, the validity of assumptions are tied to social constructivism and politics, multiple perspectives are connected to social justice, and informed actions are accomplished through creative media production. The critical media literacy framework can be viewed as containing fundamental elements of all four previous empowerment frameworks presented in this study. Potter's (2004) cognitive approach is present here; as a student moves through the cognitive development of media literacy, they are understanding the concepts of social constructivism, semiotics, and positionality. The inquirybased approach is recognizable here through the use of critical questioning and teaching in a collaborative environment. New media literacies can be integrated into all steps of this frame since they are based on social and cultural competencies that advance students towards the use of participatory critical thinking skills (Jenkins et al., 2009). Finally, media production is addressed directly through the promotion of knowledge generation via 
production over mere knowledge consumption (Brookfield, 2012; HeeYoung Kim et al., 2016).

The inclusion of these concepts, however, does not mean that the critical framework is representative of all approaches to media literacy. The frame has its detractors as well, with some suggesting that critical media literacy should actually be placed within the protectionist paradigm due to its focus on social justice and identity politics (RobbGrieco \& Hobbs, 2013). It can be understood how addressing student vulnerabilities and viewing media as being an unjust social system can be viewed as protectionist, however, this frame encourages students to question social injustices through critical questioning and dialogue.

A study of the teaching of critical media literacy in a K-12 setting found that use of this pedagogy was only successful when students were empowered through active participation in the instruction (Gainer, 2010). The results emphasize the importance of collaboration among instructors and learners as co-facilitators in the learning taking place through critical pedagogy. Ultimately, this framework situates students as active change agents within their media literacy instruction (HeeYoung Kim et al., 2016) by empowering them to address social injustices found in the media through a critical interpretation of messages.

\section{Conclusion}

Instruction in media literacy has been shown to lead to transformative learning and a critical understanding of the role of media in our society. The relevance of this pedagogy is imperative as media and digital communication technologies become increasingly indispensable to daily life. Regrettably, media literacy instruction has not 
matched the momentum of this societal priority and remains on the periphery of American education. Due to national policy backing such as the Common Core State Standards and oversight from the Department of Education, media literacy pedagogy is becoming more commonplace in elementary and secondary education by means of an integrative approach. Post-secondary instruction of media literacy in the U.S. is much less prevalent, however. Lacking policy oversight, incorporation into university curriculum is infrequent and scattered across varying pedagogical approaches and disciplines. The isolated nature of media literacy implementation in higher education settings has also led to discrepancies in its instruction, learning outcomes, and assessment tools.

The frameworks through which media literacy is taught in post-secondary education have shifted over time, with a consensus of scholars designating empowerment frames in preference to protectionism. This scholarly review established the definition of media literacy as the ability to access, analyze, evaluate, and create messages in a variety of forms (Aufderheide, 1993), and indicated how that definition was revised over time into protectionist frameworks such as Buckingham's (2003) modified framework and the ideological analysis approach. Based on the philosophies of Dewey (1938) and Friere (2010), empowerment frameworks have ultimately become the preferred method through which to teach media literacy. Common themes across all empowerment frameworks include elements of critical thinking, critical reflection, inquiry-based instruction, group and project-based learning, and creative media production.

As stated previously, one of the most crucial barriers hindering the advancement of media literacy education in U.S. higher education is its inadequate policy oversight. 
This lack of policy support has contributed to inconsistencies in learning outcomes, instructional approaches, and assessment. The next section of this scholarly review explores media and educational policies in the U.S., and then compare them to the policy environment in other nations.

\section{Media Literacy Policy}

Comparisons of U.S. media literacy educational policy to other countries such as the United Kingdom, the European Union member states, and Canada is one of contradiction. These countries, along with many others, have passed legislation and enacted policies ensuring the inclusion of media literacy education for their citizens (Buckingham, 2009; European Union, 2007). The U.S., while being the global leader in media production and distribution, has instead situated media literacy on the fringes of the education system (Mihailidis, 2008). Superficially, this difference in approaches appears to be due to the U.S. being the largest exporter of media and entertainment in the world; the European Union, for instance, is ensuring that external cultural influences are moderated so their citizens are able to critically consume and understand their interactions with this imported media (European Union, 2007). However, it goes further than a simple fear of cultural assimilation, and instead is more deeply rooted in the different ideologies upon which policies are based. This dichotomous perspective of dominating worldwide media yet taking an indifferent stance to its inclusion in the curriculum in the U.S. is primarily due to issues of language and the deregulatory approach of current policies that focus on individual freedom over regulation (St. John et al., 2013).

Media literacy has become more prevalent in recent years, growing in parallel to concepts such as technological literacy and digital literacy (Buckingham, 2009; De Abreu 
et al., 2014). The misconceptions arising from the intermingling and substitutional use of these three different forms of literacy are creating policy challenges based on the language utilized (De Abreu et al., 2014). While educators recognize the need for teaching students how to best use new technologies, there is an imbalance in understanding how the concept of critical media literacy can be taught in conjunction with these new digital tools (De Abreu et al., 2014). The current media environment is based on continual technological change, however, the focus should not solely be on the technology itself, but the "cultural, social, economic and political" (Buckingham, 2009, p. 13) implications as well. Buckingham (2009) suggests that by taking a loose interpretation of the language used for these different types of literacy, policymakers are shifting media literacy education to the background, attaching it as a subset to digital literacy. While there are some positives for linking these different literacies together, this shuffling of the language leads to confusion about the goals and implementation of media literacy education (De Abreu et al., 2014; Mihailidis, 2008).

The framing of how media literacy is inadvertently included in U. S. educational policies as a negligible component of a vague term such as digital literacy is important when viewing these policies in comparison to more comprehensive polices in other countries. Nations such as Canada, the United Kingdom, the Nordic countries, and other European Union member states have developed explicit policies that guide the teaching of media literacy in both PK-12 and post-secondary educational settings (Andersen et al., 2013; European Union, 2007; Lundgren et al., 2013). These policies also differentiate media literacy from digital or technological literacy to varying extents (Buckingham, 2009, p. 13). The reasons for the differences in education policies in the U.S. compared 
to these other countries is due to the deregulation of media industries and the neoliberal policy environment in the education system that emphasizes technological determinism (De Abreu et al., 2014; St. John et al., 2013).

\section{Media Literacy Education Policy in the U.S.}

Media and education have historically been segmented regarding regulation and policy in the U.S. Media is overseen by the Federal Communications Committee (FCC), a government agency that enacts media policy and regulations. State and local governments have authority over education, with additional policy recommendations and oversight originating in the Department of Education, a cabinet-level department of the federal government. These departments are philosophically unstable in the long term and are under differing levels of pressure as leadership changes occur in the executive and legislative branches of government. Public sentiment and corporate influence can also command significant sway in the decision-making processes of both agencies.

Media literacy as a policy concern has emerged from multiple sectors, including education, professional, community-based, and media arts (Silverblatt, 2014). This section of the scholarly review focuses on two key policy areas. An examination of how the politics of media deregulation have influenced media policy first takes place, followed by a look at the national policies regarding educational technology and media literacy, more specifically the exclusion of media literacy in institutions of higher education.

Media policy. An approach based on deregulation and neoliberal ideology has defined the U.S. media industry since the 1980s (Freedman, 2008). With minimal governmental control over media ownership, the U.S. public has little choice but to decide on its own how to best consume and interact with the media. The key to this deregulation is 
not to ensure a free market economy, but rather to ensure the safety and success of private interests (McChesney, 2003). This differs from countries in Europe, for example, who often enact media policies to avoid cultural homogenization due to effects of globalization (Buckingham, 2009). Since media in the U.S. is already domestic in nature, U.S. policies protect the media industry so it can maintain its global dominance. On the whole, this deregulation protects private business in lieu of the greater public interest (Buckingham, 2009; McChesney, 2003).

Media policy in the U.S. is often a reaction to issues created by mass-mediated communications, as well as the notion of media being societal structures that have significant political and cultural implications (Freedman, 2008). The reaction originates from an attempt to understand how forms of communication affect the cultures that support them. This has led to media policy that, in theory, both protects the media forms seen to be key components of democracy, as well as encouraging the involvement of citizens in the political process (Freedman, 2008). To make informed judgements, it is crucial for citizens to have unobstructed access to a diverse array of information. This is one of the performance goals of the FCC, as conveyed in their most recent strategic plan:

The FCC will continue to examine its current rules and make changes as required to accommodate the policy goals of competition, diversity, and localism within the evolving media landscape. Media plays a powerful role in the democratic process, as well as in shaping perceptions about who we are as individuals and as a nation. In order for broadcasters to best serve the public interest, we must pre- 
serve an avenue through which diverse voices can be heard and the interests of local communities can be served. (Federal Communications Commission, 2015, p. 9)

The extent to which the government has pursued these intentions is inconsistent with the reality of a deregulated media industry. Regardless of this disparity, the genuine existence of media policy can still be rationalized because of the, "desire to maximize the provision of different voices and perspectives in and through the media" (Freedman, 2008, p. 8).

Policy can be viewed as both a political and social action (Bardach, 2012), concerned with benefiting the lives and well-being of the public through the creation of systems to actualize these goals (Freedman, 2008). Neoliberalism has emerged as the dominant perspective through which media policy is currently structured and critiqued. Neoliberalism is described as a philosophy that gives utmost importance to profit as the basis for democracy and consumption as the preferred form of citizenship (Giroux, 2001). The neoliberal agenda in media policy frames the public as customers, commerce as the priority, and concentrated ownership through media conglomerates as the ideal structure (McChesney, 2003). In the context of policy, neoliberalism is inextricably linked with the deregulation, privatization, and marketization efforts seen in media and entertainment since the 1980s (McChesney, 2003; Freedman, 2008).

Deregulation, or the limiting of government to administer business, is the most distinguishable component of neoliberal media policy in the U.S. Deregulation asserts that regulation should be generated through the influence of market forces. Deregulatory processes began in earnest during the Reagan administration, with an aim of limiting the 
power of the FCC and lifting regulations governing ownership and content (Freedman, 2008). Increased deregulation continued through the 1990s, with media conglomerates lobbying for further commercial interests. This deregulation eventually took hold in other parts of the world, including the United Kingdom and western Europe, as well as nations in eastern Europe following the collapse of the Soviet Union (Freedman, 2008). These changes in regulation were a political action exhibiting a preference for profitability over mere disengagement of the state from the policymaking process. As mentioned earlier, one outcome of this deregulation was the consolidation of media ownership into a limited number of large corporations. This form of ownership is dictated by consumption practices, greatly reducing the diversification of content offered through all media platforms.

In this deregulated policy environment, the public is expected to have the capacity to inform themselves in a technologically challenging setting and manage any risks that may arise. Arguments can be made for or against this approach; nevertheless, it is the current situation in regard to the American media industry, with no signs of these policies changing in the future. Media literacy does, however, advocate the prospect of citizens becoming competent, well-informed decision makers (Lunt \& Livingstone, 2012). As technology progresses, a more complex consumer landscape is emerging. People must become literate in the specificities of the media landscape to take advantage of the new opportunities afforded by technological change, while simultaneously being aware that the risks are elevated by the increased possibility of making poor decisions through more complex media interactions. 
Although media literacy can alleviate many of the issues that are passed on to consumers because of neoliberal policies, media literacy education policy cannot be restricted to the ideologies that have informed media policy over the last four decades. Neoliberal ideology encourages self-interest, consumerism, and individualism (Giroux, 2001), which are altogether at odds with a critical pedagogy concentrated on alliances, empathy, and active citizenship. Media literacy as a fundamental component of educational policy should be based on the need for students to acquire competencies for navigating our participatory, mediated society through an engaging, scalable, relevant, and sustainable practice (Livingstone et al., 2014). Policymakers do not, however, create policies that would promote this literacy. Instead, policies are enacted that encourage media use in educational settings to take place through a neoliberal pedagogical framework. The next section explores how technological and digital literacies have emerged from this neoliberal rhetoric.

Education policy. American education is unique when compared to many other countries in that it is decentralized, with local and state governments having the most control and contributing the majority of funding. There has been a significant increase, however, in the role of the federal government in education over the past few decades (Harris et al., 2011). This dynamic has created a noticeable tension between central authority and local control (Malen et al., 2011). The most significant change driving education policy over the past two decades is the role of marketization, which was guided in part due to the increased role of state and federal governments, shifts in racial politics, perceived failures of the public education system, and the general shift to marketization and privatization in American culture (Harris et al., 2011). 
This expansion of federal influence has coincided with the increased use of technology in education. As technology becomes more prevalent, for example smartphone ownership among students, it becomes more difficult to exclude it from the classroom. While arguments can be made against the presence of media and technology in the classroom, the truth is that these technologies are now ubiquitous in our society. Because technology and media play such an important role in everyday life, their inclusion in learning is necessary as a core subject. Instruction in media literacy is more important now than ever to ensure a critical understanding of these new media and technologies. This type of learning is also crucial in combatting neoliberal theoretical concepts of selfinterest, marketization, and privatization (Giroux, 2001) that are typically connected to these technologies.

The inclusion of digital technology rhetoric in U.S. educational policies has been used in the past to promote the individual skill building nature of the education experience (Buckingham, 2007; De Abreu et al., 2014). Conflict arises when comparing the frameworks through which media literacy is taught, with a focus on group collaboration, creativity, active citizenship, and critical media production (Buckingham, 2009; Hobbs, 2010; Morrell et al., 2013), to current educational technology policy that aims for the efficient standardization of learning in order for students to gain proficiency in specific skills (De Abreu et al., 2014). By focusing policy on practical technological skills with an end-goal of success in the job market, it is difficult to integrate the learning styles necessary for the proper instruction of media literacy into the classroom.

Positive steps have been taken recently, however, to include fundamental components of media literacy into technology policy in the U.S. Examination of the language 
used by policy-making institutions such as the Department of Education shows the recent change from a skill focus to a more critical application of technology:

To remain globally competitive and develop engaged citizens, our schools should weave 21 st century competencies and expertise throughout the learning experience. These include the development of critical thinking, complex problem solving, collaboration, and adding multimedia communication into the teaching of traditional academic subjects. (Office of Educational Technology, 2016, p. 8)

This most recent national educational technology plan shows that momentum is shifting towards the types of pedagogy buttressed by media literacy; personalized learning, project-based learning, creative production, learning outside the classroom, and transformative learning opportunities are all emphasized (Office of Educational Technology, 2016). Despite these ideological developments, the inclusion of media literacy education into national policy still has a long road ahead. For instance, the term media literacy is not mentioned once in the Department of Education's entire educational technology plan. By continuing to disregard media literacy, the federal government is ensuring that it will continue to be a misunderstood competency without clear goals or definitions, and that media is seen simply as a tool rather than an important pedagogical requirement.

Renee Hobbs (2010) created a set of recommendations to establish media literacy as a key component of education in the U.S. This plan includes local, state, and national initiatives that would have strong implications for future policy development through the support of community-based media literacy projects, bolstering media literacy's inclusion in teacher education, the development of assessment tools, and national stakeholder en- 
gagement. Although this proposal was written in response to the previous national educational technology plan published in 2010, the recommendations and the challenges that must be addressed for these initiatives to be successful are still relevant in light of the updated educational technology plan. While we are seeing a paradigm shift in technology policy towards more critical thinking, the same issues of a lack of media literacy inclusion in policy and the ambiguous use of language for different types of literacy allows legislators to keep media literacy education outside of the national policy discussion.

In order for media literacy to be included in the policymaking process, five challenges must be overcome to ensure success: (a) thinking outside of the paradigm of digital tool access equaling a critical understanding of said tool, (b) understanding the risks associated with current media technologies, (c) a continual update to the understanding of the concept of literacy, (d) reinforcing the ability of students to gauge the credibility and quality of media messages, and (e) the inclusion of current events coverage into educational settings (Hobbs, 2010). As stated earlier, recent federal education plans have focused on technology access, with an underlying assumption that being able to use any piece of technology automatically equals a critical understanding of it. These expectations parallel the communication gap present in the concept of digital natives versus digital immigrants, and do not consider differing skills and understanding among students of differing socioeconomic backgrounds.

Finally, another major development stemming from the exclusion of media literacy in U.S. education policy is a growing disparity between how students are interacting with media at home and in the classroom (Buckingham, 2007). While independence and critical participation are encouraged as youth navigate these new mediums on their own, 
they are expected to adhere to a pedagogy based on standardization and isolated skillbuilding in the classroom (Buckingham, 2007; St. John et al., 2013). This discrepancy has led to more educators not being prepared to include curriculum based on new digital tools being taught through innovative learning styles (De Abreu et al., 2014). This imbalance will only continue to grow as more pre-service teachers are neglected media literacy instruction during their post-secondary studies.

It is a momentous challenge for media literacy policy to exist in a neoliberal system that relies on marketization to create a workforce with basic technical skills that can compete in a globalized marketplace. Media literacy instruction contradicts the individual, market-driven, and privatized focus of policy surrounding both media and education. A method of working within a neoliberal policy environment must be discovered to develop active citizens with a critical understanding of media and technology. Viewing how media literacy is incorporated into educational policy in other nations can guide how media literacy education can gain a foothold in the U.S.

\section{Global Media Literacy Education Policy}

At odds with the shortcomings of enacting cohesive policy in the U.S., countries such as Canada, the United Kingdom, and member states in the European Union have successfully established formal media literacy instruction in various educational settings. The European Union, while being similar to the U.S. federal government due to a lack of legitimate authority it holds over member states, has nevertheless witnessed substantial achievements through policy initiatives and research. All member states are strongly encouraged to enact media literacy programs in their schools for citizens to better understand the role, possibilities, and challenges that come with media use in a digital economy 
(European Union, 2007). The United Kingdom, Canada, and the Nordic countries all particularly stand out as having instituted successful media literacy policies through varying means. All of these nations have also seen significant media deregulation over the last thirty years, though to a lesser extent than the U.S. Simultaneously strengthening domestic media industries through deregulation while limiting outside competition, namely American media, has been the guiding principle in these countries.

An understanding of media ownership and industry deregulation in different countries is crucial, in terms of media literacy, as ownership patterns influence both what content is presented by the media and how that content is presented (Silverblatt, 2014). When viewing media literacy educational policy in countries foreign to the U.S., there must be an awareness of the media ownership patterns in that nation. The three types of media ownership are state, public, and private ownership; each system has its particular form of influence on the construction of media messages. State-owned media tend to promote governmental agendas and limit alternative expression, while public media are funded through a system of public financing, allowing for autonomy from governmental oversight and an increase in the diversity of media producers and messages (Silverblatt, 2014). Private ownership, which is the predominant form of media ownership in the U.S., is based on a market-driven economy focused on boosting profits for shareholders (McChesney, 2003). In the interest of maximum profits, the number of media companies in countries with high levels of private ownership has led to a small number of large corporations controlling nearly all media content (Silverblatt, 2014). Private ownership impacts media in multiple ways; content tends to be homogenous, the status quo is reinforced, programming is seen as a commercial product, production and content is heavily 
influenced by advertisers, and conflicts of interest abound (Silverblatt, 2014). Although the Internet has helped to infiltrate the obstructions that come with the marketization of media content, there is currently a proliferation of various regulations being proposed in most developed nations around the globe that attempt to limit net neutrality and bring new digital communication tools into the fold of privatization. In addition, it is striking to recognize that countries who have had the most success in executing media literacy educational policy also tend to favor public media ownership.

Although different countries possess their own individual circumstances as to how they approach the teaching of media literacy, there are some common areas of agreement of the basic concepts that define media literacy. These shared conceptual foundations include the notion that media literacy is a critical thinking skill, that it gives citizens the tools to make independent decisions regarding media consumption and interpretation, that media are constructions of reality, and that media literacy itself is an apolitical competency (Silverblatt, 2014). When comparing national media literacy education programs in different countries, considerations must be made as to any distinctive features of a nation's culture that might influence the teaching of media literacy (Silverblatt, 2014); cultural context can inform both the theoretical frameworks and principles through which media literacy is taught in these different locations. Numerous countries have excelled at advancing media literacy as a substantive educational policy; places such as Australia, Brazil, Germany, Hong Kong, Japan, and many nations in the Middle East have developed robust policies and pedagogical frameworks particular to their individual circumstances. 
In the interest of the scope of this study, only a small number of countries and multi-nation collectives which contain similar aspects to the education and media environment in the U.S. are being considered, with an overarching goal of informing future policy development in the U.S. This section first explores media literacy educational policy in the European Union, followed by a survey of media literacy instruction in the United Kingdom and Canada. Finally, a look at the successes and failures of the pedagogical approaches for media literacy in the multi-nation collaborative association of the Nordic countries in northern Europe takes place.

Media literacy policy in the European Union. Media literacy policy in the European Union hinges upon social and political objectives, aiming for the strengthening of a common identity among E.U. member states, as well as benefitting how these nations relate to one another in a constructed, inclusive politico-economic alliance (Sarikakis \& Sarikakis, 2007). Policy establishes a shared culture as the foundation of the E.U., creating a European experience where all members can equally take part in a dialogue as citizens of a single political body. The onus for implementing this sense of shared identity is increasingly falling on digital media and communication technologies as they continue to advance concurrently with the concept of a Europeanized community (Papathanassopoulos \& Negrine, 2011). While media policy in the E.U. simultaneously discourages foreign influence, the reinforcement of this communal culture often leads to an isolated media literacy policy (Lunt \& Livingstone, 2012).

It is only in the last decade that media literacy has been able to overcome obstacles such as political marginality and disagreements over protectionist and empowerment paradigms (Rosenbaum et al., 2004) to become a significant policy issue not only in the 
E.U. itself, but also among its member states. Media literacy is currently an important policy topic among E.U. governments, with many citing its relevance as a tool with which to engage society through economic, informational, and democratic means (Lunt \& Livingstone, 2012). Protectionist versus empowerment framework debates have also been overcome, shifting from simply trying to protect vulnerable youth from the effects of media, to empowering all citizens, both youth and adults, to be innovators in today's digital culture (Buckingham, 2007; Lunt \& Livingstone, 2012). Scholars in Europe have also been successful in advocating for a highly informed citizenry through participatory, critical learning in lieu of traditional definitions of media literacy (Lunt \& Livingstone, 2012).

The European Union has allocated numerous resources towards creating comprehensive media literacy policy for its nation states. E.U. media literacy policy focuses on critical engagement and pragmatic utilization of media (European Union, 2007). Current E.U. media literacy policy is founded on a 2007 committee report that concentrated on defining a European interpretation of media literacy in a time of newly developing digital technologies. The report highlighted six competencies; these include (a) comfortably interacting with all existing media forms, (b) the active use of media through digital communication and entertainment technologies with a focus on intercultural dialogue, (c) taking a critical approach to media in regards to the accuracy and quality of content, (d) the creative use of media through current digital distribution channels, (e) an understanding of the difference between media ownership and media pluralism, and (f) the awareness of copyright issues to ensure a culture of legality when consuming and producing media content (European Union, 2007). The definition of media literacy as adopted by the E.U. 
stresses the critical components in a more forthright way than previous ones originating from the U.S., such as Aufderheide's (1993) definition developed at the Aspen Institute conference. The report also highlighted the current inadequacies in media literacy assessment (European Union, 2007). The overall view of media literacy in Europe is founded on the need for citizens to better understand the cultural and economic aspects of the media (Koltay, 2011).

Critical engagement is the focal point of this report, stemming from the need to raise awareness of exposure to foreign media and ensure cultural control. The highlighting of an understanding of the differences between media ownership, i.e., deregulated American media, and media pluralism, i.e., the presence of a range of perspectives in European media outlets, also shows the E.U.'s struggle to compete with the U.S. and maintain cultural control of media. The concept of media pluralism is not unique to the European Union; however, it is vital to the concept of being a European citizen and reinforcing the legitimacy of the E.U. (Papathanassopoulos \& Negrine, 2011). This pluralism is crucial to European media if they are to prove their importance in a transnational democratic alliance.

The report also focused on three best practice areas in the context of digital media: media literacy in commercial communication, media literacy for audiovisual media, and media literacy for online interactions (European Union, 2007). Media literacy for commercial communication again takes a critical approach, stating that media should be presented without biases or the use of stereotypes to convey messages (European Union, 2007). The report focuses not only on consumers but on the media industry itself, making a compelling argument for transparency in financial dealings and following a code of 
conduct in the production of media messages. Media literacy for audiovisual media promotes better awareness of past European media traditions, learning creative production skills, and an understanding of copyright from both the perspective of the consumer as well as from a business standpoint (European Union, 2007). Finally, media literacy for online communication focuses on the need for media literacy competencies in relation to current economic, cultural, and technological innovations. These competencies include empowering citizens to critically assess online content, extend production skills and creativity to the online environment, and ensuring equal access to all citizens regardless of education level, socioeconomic status, or disability level (European Union, 2007).

This report is a cornerstone of media literacy policy development in the European Union by advancing various media literacy initiatives and programs with the simultaneous rise of digital media and communication technologies over the past decade. The enactment of an overarching policy from a multi-union collective such as the E.U., that can then be imparted to member states to deploy within their particular contexts, is an appropriate example for how the U.S. Department of Education could establish media literacy education policy. The next international policy example of this scholarly review, that of the Nordic countries, offers another relevant example of a multi-state collective enacting policy in an environment comparable to that of the U.S.

Nordic media literacy. The Nordic countries in northern Europe, consisting of Denmark, Finland, Iceland, Norway, Sweden, Greenland, the Faroe Islands, and the Åland Islands, stand out as another intriguing participant in the global media literacy movement when viewed in the context of policy development in the U.S. These coun- 
tries have been joined in a formal collaboration since the early 1950s, with common beliefs and a shared cultural identity (Lundgren et al., 2013). Unlike other European countries, the Nordic countries have a decentralized educational system, similar to the U.S. Contrasting from U.S. policy, both education and media are controlled by the state and freely available to all citizens (Mihailidis, 2005).

The distribution of educational governance is seen as an essential rationalization for regional collaboration. Media and information literacy in these countries is administered through the Swedish Media Council, which is a government agency responsible for empowering youth to become critical media users (Swedish Media Council, 2016). Policy development and research are coordinated through this organization among all the individual Nordic countries, guided by a circumscribed neoliberal agenda not wholly dissimilar to the U.S. (Freedman, 2008). A noteworthy difference among the Nordic countries, when compared to other European nations, is that these countries have the lowest numbers of media consumption on the continent (Papathanassopoulos \& Negrine, 2011).

The Nordic perspective on media literacy is one shared with the European Union, as a type of learning with, "the potential to redefine active citizenship and democratic participation and advance the competence to cope autonomously and critically with the communication and media environment established within, and as a consequence of, the information society" (Lundgren et al., 2013, p. 62-63). Pedagogical practices are contributed from all countries, with scholars from Denmark, Finland, and Norway making significant contributions (Erstad, 2010; Lundgren et al., 2013; Tufte, 1998). An underlying 
theme of practical collaboration and sharing of best practices defines Nordic media literacy instruction, which includes the distribution of teaching resources and research findings among these nations (Lundgren et al., 2013).

The fundamental concepts found in Potter's (2004) cognitive approach guide the teaching of media literacy, emphasizing it as a lifelong learning proficiency; containing multiple dimensions such as cognitive, emotional, aesthetic and moral elements; and giving citizens more control over their interpretation of media texts. Learning takes place through experiential, problem-based, and collaborative methods in an attempt to ensure transformative learning among all students (Lundgren et al., 2013). Nordic university faculty especially recognize the importance of the inclusion of media literacy instruction in higher education contexts (Mihailidis, 2005). Like the present situation in the U.S., however, media literacy is currently viewed as being at an ideological crossroads. Even though this kind of learning is prevalent when compared to the U.S., questions about the contexts in which it should be taught and the ability to turn knowledge into practice in formal and non-formal environments continues to challenge the position of media literacy as a pedagogical necessity among the public (Lundgren et al., 2013).

The Nordic community represents a compelling representation of individual countries, as well as a multinational collective, enacting media literacy policy through the guidance of the European Union, yet still questioning the practicality of its achievements. As observed in the European Union, basing policy decisions on maintaining cultural hegemony can often lead to an isolationist concept of media literacy (Lunt \& Livingstone, 2012). An impending intellectual shift is forthcoming in these countries, aiming to rein- 
force media literacy as a core component of lifelong learning and to isolate it as an educational subject matter. A change of context for media literacy education is seen as key; examples of ways in which media literacy can become more successful in these countries include placing media literacy as a central component in applied education and professional development, strengthening the general public's interest in media competencies, strengthening the links between research and local education districts, the promotion of UNESCO's media literacy curriculum for pre-service teacher education, and supporting equal opportunities regarding gender, socioeconomic status, and the multicultural societies in these countries (Lundgren et al., 2013). The scholarly review will now shift focus to an example of media literacy education being established as a component of media policy rather than education policy in the United Kingdom.

Media literacy in the United Kingdom. Media literacy policy in the United Kingdom is based on the 2003 Communications Act, which conferred regulatory power for old and new media to the Office of Communications (Ofcom) (Parliament of the United Kingdom, 2003). The legislation not only handed regulative control to Ofcom, but also assigned the unprecedented task of promoting media literacy as a necessary competence among the general public (Bazalgette \& Silverblatt, 2014; Lunt \& Livingstone, 2012). Although the inclusion of media literacy was an integral component of the act, the central intention of the legislation was to lessen the regulatory power of the government over media and give greater power to market forces (Buckingham, 2009).

The U.K. has a long history of media education, and media literacy is viewed not necessarily as a subject unto itself, rather the outcome from a media education that is the, "process of teaching and learning about media" (Buckingham, 2003, p. 4). In England, 
for instance, elements of media education are included in the national English curriculum, and standalone media studies courses are also made available for secondary students (Bazalgette \& Silverblatt, 2014). Media education is already a well-established subject matter in the countries of the U.K., and media literacy can be seen as a growing element of that education. This material is taught both in standalone media studies courses, as well as a component of other subject areas in all levels of education (Bazalgette \& Silverblatt, 2014).

Ofcom defines media literacy as the ability to access, understand and create communications in a variety of contexts (Parliament of the United Kingdom, 2003), and this definition has been widely adopted among other countries (Buckingham, 2009). As in the U.S., however, media literacy is being misinterpreted as a neoliberal strategy for citizens to protect themselves in a deregulated media environment without government assistance. From one point of view, this is shifting the discussion from a protectionist view to an empowerment one, but it is also framing media literacy as a personal skill rather than a social proficiency, placing responsibility on the citizens themselves (Buckingham, 2009). Ofcom is valuing media literacy as a justification for decreasing market regulation (Lunt \& Livingstone, 2012). Another similarity to the U.S. is a recent focal shift in U.K. policy from media literacy to digital literacy, with new plans calling for a spotlight on digital participation that replaces critical analysis of media with a skills-based approach to understanding how to best use emerging digital technologies (Buckingham, 2009).

The ways in which the U.K. differs from the U.S. in terms of market deregulation and neoliberal policy ideals could appear tenuous, however, there are two significant differences: first, media literacy has become a matter of media regulation due to the 2003 
Communications Act, so that it does not rely solely on the education sector for endorsement; second, the U.K. has the ability to complement its policy initiatives with those originating in the European Union. If the U.K. government can realize the goal of a media-literate public, then arguments can be made in favor of market deregulation and a decrease in consumer protection (Lunt \& Livingstone, 2012). The ability to bridge that gap is pivotal to media literacy policy discussions in the U.K. This outlook is implying that a self-regulating, knowledgeable audience serves as rationalization to increase public selfregulation and decrease state regulation (Lunt \& Livingstone, 2012).

The problem is that these initiatives are not necessarily taking place. Instead of clarifying the definition of media literacy and demonstrating the ways in which understanding can be assessed among citizens, U.K. policy is mimicking U.S. educational policy in maneuvering focus from critical pedagogies such as media literacy to more focused, skillsbased literacies such as digital and information literacy (Buckingham, 2009). Concentrating on individual skills over social capabilities limits all forms of literacy, which require social interactions to reveal authentic social and cultural contexts (Buckingham, 2003). Attempts to disregard critical learning and creative production, both key components of media literacy, allows for political ideology to inform how citizens should act and think in a mediated society (Lunt \& Livingstone, 2012). In order to counter this approach, European Union policy is proposing media literacy assessment tools for all of its member states (Lunt \& Livingstone, 2012).

Despite a long history of media education and the inclusion of media literacy in national policy, the U.K. currently finds itself in a position comparable to the U.S. Digital literacy has become the priority, with media literacy viewed as either secondary to or a 
component of digital literacy. Digital technologies were originally understood to be merely a component of the media literacy movement in the U.K., but have now become the main impetus for the continued existence of media literacy policy and the main context through which media literacy is referenced (Wallis \& Buckingham, 2013). Recently, media literacy policy in the U.K. has been declared as being nonexistent and all but abandoned (Buckingham, 2016). Buckingham (2016) states that, "any extended educational conception of media literacy ultimately gave way to a much more reductive, functional notion of technological skill; and to the need to be seen to be doing something about online safety."

It is tempting to view U.K. media literacy policy as a failure and an excuse for the continued advancement of neoliberal principles. However, it should be viewed, in regard to this study, as an example of a well-intentioned but poorly executed policy development. Attempts to enact an educational initiative, notably one based on critical pedagogy, through a disparate policy area such as media regulation have not been successful. While there are obvious connections between media literacy and media policy, it is nevertheless a pedagogy rather than a political practice. These shortcomings can inform policy development in the U.S. through a detailed example of the inherent risks when attempting to achieve an understanding of critical pedagogy through adverse ideologies such as neoliberalism. The final international policy example, the nation of Canada, is an exemplification of the positive outcomes that are possible when national guidelines are not only in place, but also actualized.

Canadian media literacy. Media literacy in Canada stems from preceding polices originating in the United Kingdom and Australia. U.K. media literacy education is 
one influenced by class structure and an awareness of ideological differences among socio-economic structures, while Australian policy emerged as a defensive response to the influence of American media with a critical focus on media production (Andersen et al., 2013). Both perspectives are foundations of Canadian media literacy policy.

Canada is in a unique position to share its only border with the largest exporter of media and entertainment in the world, and Canadians consume more American media than domestic (Andersen et al., 2013). This acute awareness of the impact of American culture has led to a sense of detachment for Canadian citizens, helping them become more reflective and analytical about what media they are consuming. Canada was also a key player in the General Agreement on Tariffs and Trades, which led to many countries, including those in the European Union, to force an exemption from free-trade regulations that would have seen an increased deregulation and liberalization of American media in their respective nations (Freedman, 2008).

Canada is similar to the U.S. in that each province and territory is responsible for its own educational system (Duncan et al., 2014). Over the last thirty years, media literacy has become a key component of the core curriculum in Canadian elementary and secondary schools, with a strong attachment to the English curriculum in secondary education (Andersen et al., 2013; Duncan et al., 2014). Canadian media literacy sets itself apart from the U.S. with its emphasis on education. Duncan (2006) states:

Media literacy is concerned with developing an informed and critical understanding of the nature of the mass media, the techniques used by them, and the impact of these techniques. It is education that aims to increase students' understanding 
and enjoyment of how the media work, how they produce meaning, how they are organized, and how they construct reality. (p. 31)

Media literacy developed as an important pedagogical necessity due to concerns over the influence of American media, a fixation among Canadian educators with emerging digital media, and the existence of educational and professional development systems that encourage perpetual curriculum changes as technological and social developments occur (Duncan et al., 2014). By the early 2000s, all provinces had enacted policy ensuring media literacy's inclusion in secondary English courses, as well as standalone classes in select provinces (Duncan et al., 2014). Media literacy is also a required curriculum component in elementary education in all provinces (Andersen et al., 2013).

The Association for Media Literacy (AML) is responsible for developing frameworks and learning goals for media literacy education in Canada. The AML has produced two frameworks through which media literacy is taught in Canadian schools; educators use one or a combination of both frameworks to teach media literacy, depending on personal preference and the learning styles of their students. The first framework, known as The Eight Key Concepts of Media Education, was first introduced by the Ontario Ministry of Education (Duncan, 1989), and gives educators common terminology to use in media literacy instruction. These include the concepts that (a) media are constructs of reality, (b) media construct versions of reality, (c) audiences actively negotiate meaning, (d) media messages have economic implications, (e) media texts include value and ideological messages, (f) media texts have social and political implications, (g) content and form are closely related, and (h) each medium has a unique aesthetic form (Association for Media Literacy, 2016). Teachers typically utilize this framework by applying a 
few of the concepts at a time to select media texts (Andersen et al., 2013). The second framework, known as the Media Literacy Triangle (see Figure 7), organizes the eight key concepts into a graphical form broken down to three categories: text, production, and audience.

\section{Figure 7}

\section{Media Literacy Triangle}

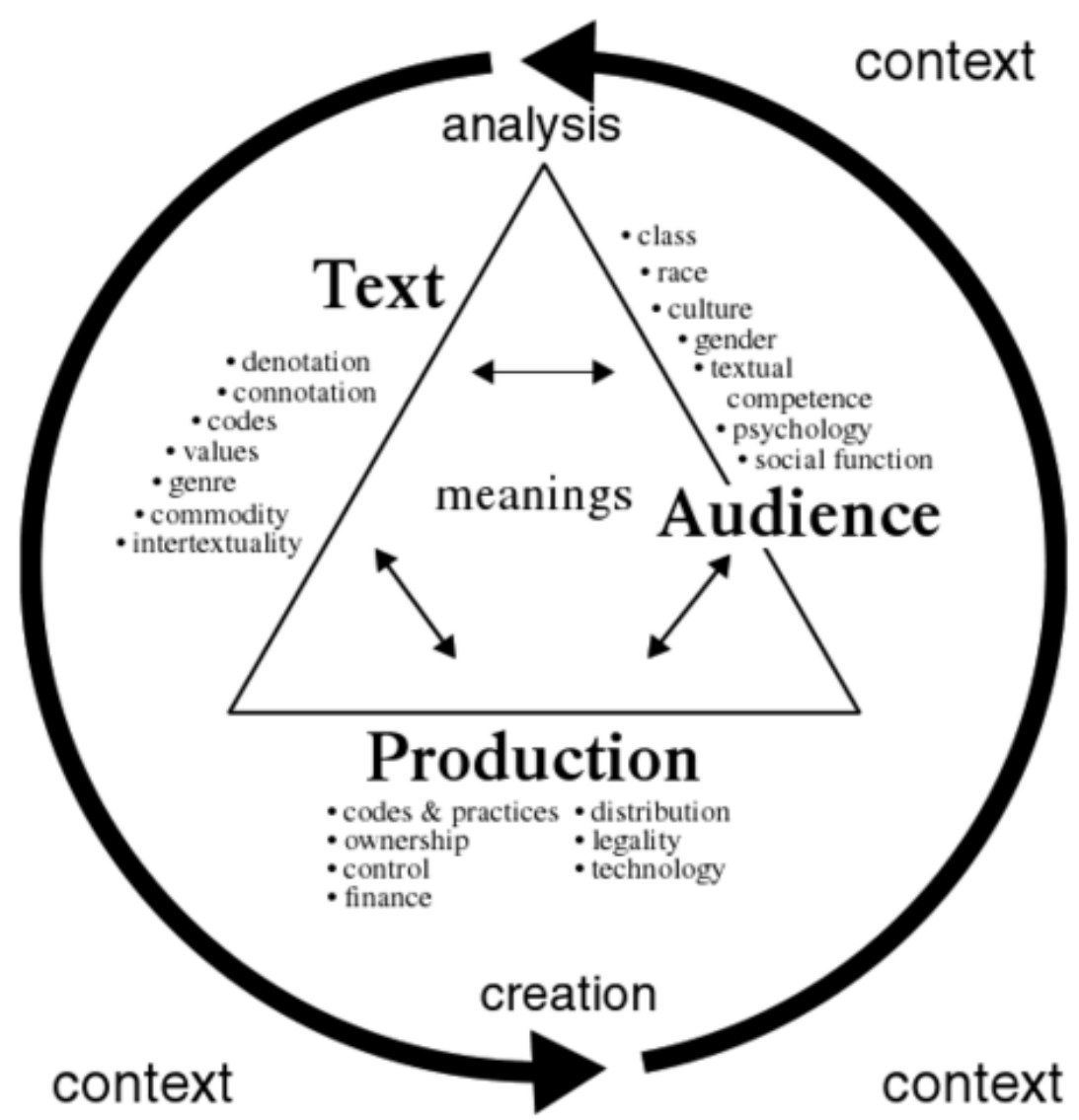

Note. This figure illustrates the three categories through which media texts can be explored. Reprinted from Formation \& Media Literacy, In Association for Media Literacy, 2016, Retrieved March 21, 2016, from http://www.aml.ca/2769-2/. Copyright 2016 by The Association for Media Literacy.

Students can utilize the triangle by placing a media text in the middle of the graphic, then exploring that text through the three different points of view (Andersen et al., 2013). 
Canadian media education also specifies nine factors necessary for the successful deployment of media literacy education. These state that (a) media literacy must be a grassroots movement with direct engagement from educators, (b) administrators must give explicit support to this type of education through mandatory inclusion in the curriculum, (c) all new teachers must understand this subject area, (d) program implementation relies upon in-service training at schools, (e) school districts are required to have consultants on hand to establish proper communication networks, (f) relevant texts and media materials must be made available to assist in this instruction, (g) a professional development network must be present across all school districts, (h) assessment instruments must be made available, and (i) collaboration is required among teachers, parents, researchers and media professionals to ensure the diverse array of skills and expertise necessary in media literacy education are successfully implemented (Andersen et al., 2013). The combination of standardized pedagogical frameworks, the inclusion of media literacy instruction across all grade levels, and policies that explicitly state the requirements for successful implementation situates Canada at the forefront of global media literacy education.

Similar to the European Union, media education in Canada hinges upon cultural studies, since media literacy is seen as a solution to understanding socioeconomic differences in the culture and how the media represents reality (Duncan et al., 2014). Creative media production is also implicit in this instruction, ensuring students are knowledgeable in emerging digital technologies found both inside and outside of the classroom. Media literacy is seen as a necessary life skill that prepares citizens to be critical thinkers and ensures they can successfully communicate in a diverse society (Andersen et al., 2013). While these characteristics are base elements of most media literacy pedagogies and not 
exclusive to Canada, media education in the country is unique, however, in its focus on audience studies that includes semiotics and critical pedagogies in the learning process (Duncan et al., 2014). Overall, Canadian media literacy education is informed by a combination of a healthy skepticism of media messages and acknowledging the importance of being able to critically consume and create communications in a society containing a diverse array of ideological perspectives.

\section{Conclusion}

Unlike the U.S., countries such as the European Union member states, the United Kingdom, and Canada have robust policies ensuring that all students are exposed to media literacy instruction. The deregulation of the media industry, as well as functioning within a neoliberal policy environment, are the primary reasons for the exclusion of media literacy in American classrooms. Educational policy has been especially affected in recent decades by the shift to marketization and privatization in the U.S. It is difficult for a critical pedagogy such as media literacy, which contradicts neoliberal media and education policy, to succeed in an environment that is focused on creating a workforce with basic technical skills with an aim to successfully compete in a globalized marketplace.

The nations considered in this scholarly review all contain comparable facets to the media and education climate in the U.S. The coalition of countries in the European Union, while being similar to the U.S. federal government due to a lack of oversight it holds over member states, has witnessed substantial achievements in media literacy education through policy initiatives and research. The Nordic countries are a meaningful example of a multi-state collective enacting local policy in conjunction with guidance from 
the European Union. The United Kingdom offers an important example of the difficulties in attempting to actualize pedagogy from a media policy standpoint instead of establishing it as educational policy. Finally, Canada has demonstrated the positive outcomes of implementing a national framework for media literacy instruction. All of the literature considered in the scholarly review, including the current media landscape, the foundations of media literacy, the educational settings and frameworks for media literacy in the U.S., and comparisons of media and education policies in the U.S. versus other nations, guides the creation of a new theoretical framework in the next section.

\section{A Pedagogical Framework for Media Literacy in Higher Education}

As suggested by Jenkins et al. (Jenkins et al., 2009), media literacy instruction should take an ecological approach in which it is acknowledged that media and audiences interact together in a complex interrelated system of technologies and culture. It has also been established that our society has developed into a participatory culture in recent decades due to of the prevalence of new media, with citizens now contributing their own original content while simultaneously consuming media and information from external sources (Chen et al., 2011; Jenkins et al., 2009). Grounded in these viewpoints, this dissertation-in-practice proposes an empowerment framework for media literacy instruction that concentrates on higher education settings. It is important to mention that while this study focuses on post-secondary settings, this framework would also be applicable in other instructional contexts such as PK-12 classrooms and adult community education.

Multiple requirements must be taken into consideration for a single framework to function within all potential contexts of post-secondary classrooms in the U.S. First, the framework must work in all possible disciplines where media literacy might be taught; 
these disciplines include media studies, teacher education, general education courses, or through an integrated approach within other subject areas. Second, the framework must be applicable in all higher education settings such as community colleges, public fouryear, and private four-year university classrooms. Third, faculty with varying backgrounds teaching across multiple disciplines must be able to utilize the framework regardless of their expertise in media studies.

This new framework, entitled the participatory ecological framework of media literacy, meets all of the requirements specific to higher education while still being grounded in ecological and participatory approaches to pedagogy. Based on an analysis of the empowerment frameworks included in this scholarly review and a synthesis of their pertinent components, the framework proposed herein is founded on critical thinking as a method for analysis, reflection, and creative production of media. The framework recognizes the definition of media literacy as being able to access media, understand and critically evaluate the different aspects contained therein, and being able to produce communications in a variety of contexts (European Union, 2007), while simultaneously being suitable for the teaching of media literacy at any level of education. Following the presentation of the pedagogical framework and its components, three example scenarios of how the framework could be utilized in a post-secondary classroom are included, highlighting the adaptability of the framework to different instructional settings and circumstances.

\section{Participatory Ecological Framework of Media Literacy}


The pedagogical frameworks presented in the scholarly review can be generally divided into two focal areas, with some negligibly discernible overlap present; the two focuses being either the analysis or production of media messages. The frameworks focusing on textual analysis include the cognitive model (Potter, 2004), inquiry-based pedagogy (National Association of Media Literacy Education, 2007), and critical media literacy as transformative pedagogy (Funk et al., 2016). Conversely, new media literacy (Jenkins et al., 2009) and critical media production (Morrell et al., 2013) have a more participatory footing in media creation. In his landmark discourse on media education, Masterman (1985) warned of the inadequacies of focusing pedagogy solely on the analysis of media texts. Similarly, concentrating instruction only on production has the potential of excluding core media literacy concepts such as ideology, politics of representation, and social justice. The framework created here from a synthesis of the scholarly review takes a unified approach by integrating the components of both analysis and production to work together in an integrative manner as a single pedagogical practice.

As previously stated, there are three key requirements for a pedagogical framework to be successful in the instruction of media literacy; those being the possibility of teaching media literacy in multiple academic disciplines, in various educational settings, and by faculty with varying levels of expertise in traditional media studies. By basing the participatory ecological framework in cultural studies and critical thinking, the subject can be taught across multiple disciplines by addressing socio-economic issues such as gender, race, class, politics, and power through the lens of the appropriate subject area. For instance, students in a teacher education course and those in a standalone general education classroom could reflect on the same media text in distinctive ways based on the 
differing backgrounds, interests, and semiotics of a particular combination of faculty and students in any given classroom situation. Similarly, the framework can be utilized in different settings such as a community college classroom or in a media studies program through a pedagogical approach particular to that environment. Finally, a proficiency in traditional media studies is not required of faculty; the framework is fluid in its adaptability and gives faculty multiple options in how they approach their teaching, whether it be in the stages of analysis, reflection, or production.

Framework components. The participatory ecological framework of media literacy takes a philosophical approach to pedagogy based on the work of Freire (2010). In this framework, Freire's (2010) guiding principles of critical dialogue, critical reflection, and action constituting a transformative pedagogy have been reconstructed into the three pedagogical approaches of analysis, reflection, and creative production of media. The framework can be visualized by a series of three concentric circles, with varying components enclosed within each, surrounding a single entity of a classroom consisting of students and faculty (see Figure 8). This framework does not occur in isolation; instead, it exists within the real-world environment that includes the media industry, policy, regulation, culture, and society.

The outer circle contains the three pedagogical approaches of analysis, reflection, and production of media. The second circle consists of the media literacy concepts of positionality, construction, and substance. The third circle includes the ten participatory methods of learning as identified by Jenkins et al. (2009) and independently verified by Literat (2014); these are comprised of play, performance, appropriation, multitasking, 
distributed cognition, judgment, transmedia navigation, networking, negotiation, and visualization. It should also be noted that the boundaries between these three circles, along with the boundaries separating the center and outside of the framework, are permeable, meaning that the environment as well as the individuals in the classroom will influence the learning taking place.

\section{Figure 8}

Participatory Ecological Framework of Media Literacy

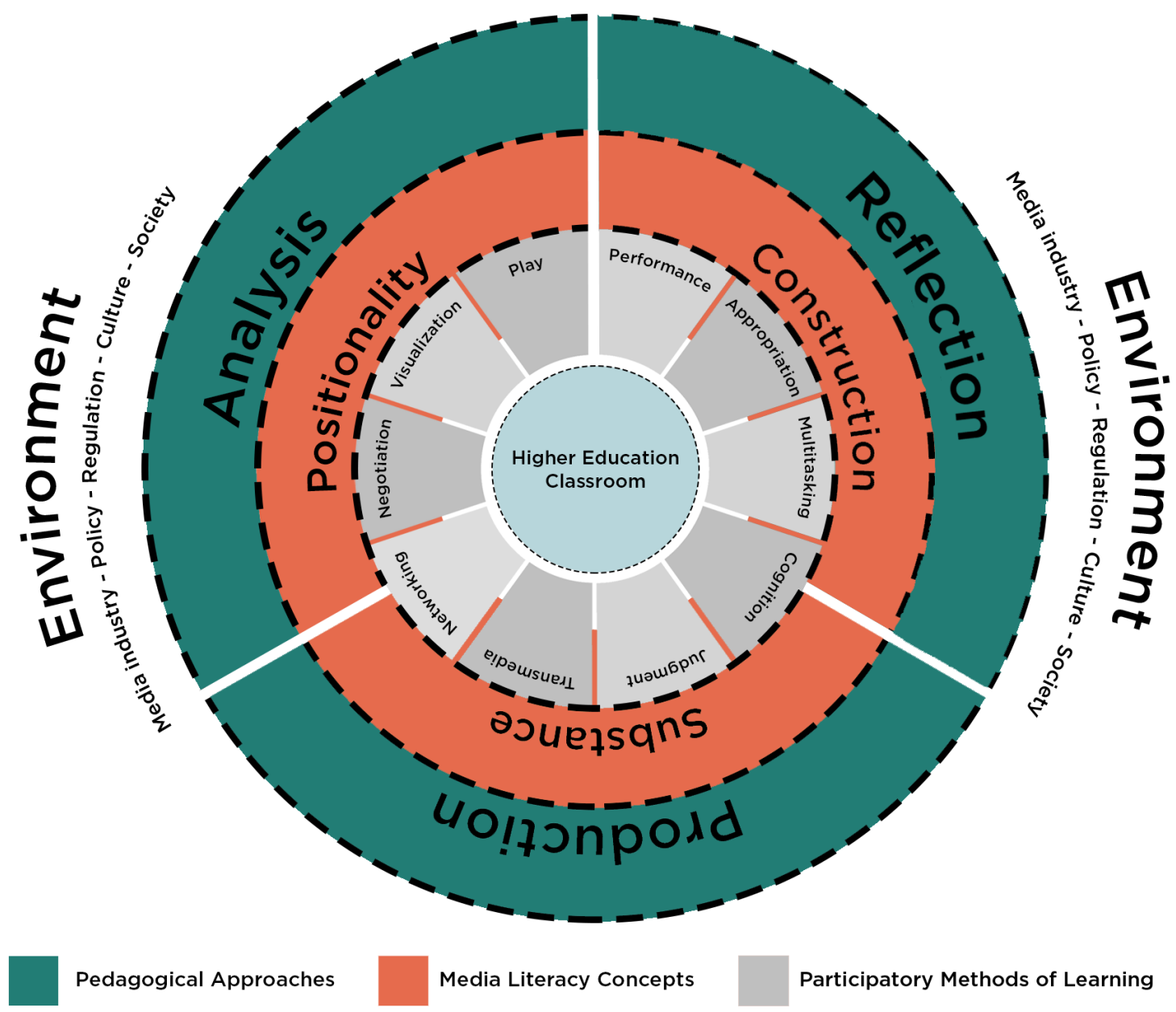

Note. This figure shows the components of the participatory ecological framework, including the pedagogical approaches, media literacy concepts, and participatory methods of learning. 
The three pedagogical approaches of analysis, reflection, and production are incorporated from the five empowerment frameworks included in this scholarly review. The framework allows for teachers to merge the three pedagogical approaches in any possible combination in their curriculum. As stated previously, the teaching of media literacy must not focus solely on the analysis or reflection of media messages, or conversely only on media production (Masterman, 1985). The three pedagogical approaches of this framework are viewed as being of equal importance and are not sufficient in teaching media literary concepts without complementing one another. While a single pedagogical approach could be employed in a segment of a larger, more comprehensive lesson, the ultimate intention of utilizing the participatory ecological framework is to consistently employ all three pedagogical approaches equitably. Again, while these three approaches are based on Freire's (2010) principles of critical dialogue, critical reflection, and action, they should not be viewed as a sequence of steps to transformative learning; rather they are independent approaches that can be taught collectively as needed based on the intentions of the learning outcomes.

The second concentric circle, containing the three core media literacy concepts of positionality, construction, and substance, are again synthesized from the five empowerment frameworks presented in the scholarly review. Positionality includes media literacy components such as audience (Funk et al., 2016), authorship, impact, interpretations, and response (National Association of Media Literacy Education, 2007). Construction consists of production, social constructivism, language and semiotics (Funk et al., 2016), purpose, and techniques (National Association of Media Literacy Education, 2007). The 
final media literacy concept of substance includes issues of politics of representation, social justice (Funk et al., 2016), content, context, and credibility (National Association of Media Literacy Education, 2007). The three media literacy concepts in this framework can work independently of one another or in any combination, and also separately or together through any of the three pedagogical approaches.

The third concentric circle includes the ten participatory methods of learning as identified by Jenkins et al. (2009) and confirmed through research conducted by Literat (2014). Specifically, these learning methods include play, performance, appropriation, multitasking, distributed cognition, judgment, transmedia navigation, networking, negotiation, and visualization. Like the media literacy concepts included in this framework, the participatory methods of learning can be used autonomously or collectively in any combination with all three pedagogical approaches, as well as include any of the three core media literacy concepts. It should be noted that while the intent is for any of the ten learning methods to work with any of the pedagogical approaches and media literacy concepts, it is evident that some combinations would work together more successfully than others; for instance, participatory learning methods such as play and appropriation perhaps more readily combine with the production approach than analysis or reflection.

Potter's (2004) cognitive model of media literacy, while not directly incorporated into this framework, is nevertheless important in guiding its construction. Upon entering higher education, students will typically fall on the continuum of media literacy development at approximately the stages of developing skepticism or intensive development (Potter, 2004), depending on the individual. The participatory ecological framework supports continued advancement along the continuum through critical analysis, reflection, 
and production. As students are exposed to the participatory learning methods of media literacy concepts through various pedagogical approaches, they will develop experiential exploring, critical appreciation, and social responsibility (Potter, 2004) through searching for alternative narratives, understanding the contexts of media messages, and discerning how individual choices can affect society as a whole.

The framework also aligns with Morrell et al.’s (2013) model for critical media production that requires learning to be active, authentic, participatory, and empowering, even though production is only one of the three recognized pedagogical approaches. Active and authentic learning takes place when the framework is utilized in a way that ensures students are accomplishing tasks that have personal meaning and significance in the real world. Participatory learning is taking place within a collaborative community of both similar and dissimilar peers, which also includes faculty members. Learning taking place through the framework is also empowering by giving students the ability to enact change outside of formal learning environments through acquiring media literacy competencies as they move along Potter's (2004) continuum of media literacy development.

Examples of the framework in action. With the components of the participatory ecological framework of media literacy firmly established, as well as an explanation of how the synthesis of concepts found in the scholarly review informed its creation, it would be helpful to demonstrate how this framework could be utilized in a real-world classroom. What follows are three example scenarios of how the framework can be used in any higher education class that includes the teaching of media literacy concepts. It should also be noted that the framework is not limited solely to post-secondary education, as it can be implemented in any educational setting since its success is based as much 
upon independent factors like the external environment and a particular classroom setting as it is on pedagogical approaches, media literacy concepts, and participatory methods of learning. As these examples are illustrated below, it will be evident how differences in the environment, classroom setting, and individuals within that classroom can affect the pedagogical approaches and learning outcomes.

Example scenario \#1. The first working example of the participatory ecological framework of media literacy takes place in a community college in the southwest U.S. The class is an introductory general education course required of all first-year students, titled Introduction to Digital Media and Communication. The semester in which this course takes place is during a major election cycle, with election day less than three weeks away.

The instructor has decided that the day's classroom activity is to analyze the election messages originating from two candidates vying for one of the senate seats in the state where the community college is located. Utilizing the participatory ecological framework of media literacy, the instructor has determined the classroom activity will focus specifically on how these messages are constructed; the students will be using the participatory learning methods of judgment, negotiation, and transmedia navigation to better understand the specifics of how the messages were created (see Figure 9). Again, construction of media messages consists of issues relating to production, social constructivism, language and semiotics (Funk et al., 2016), purpose, and techniques (National Association of Media Literacy Education, 2007). 


\section{Figure 9}

\section{Example Scenario \#1}

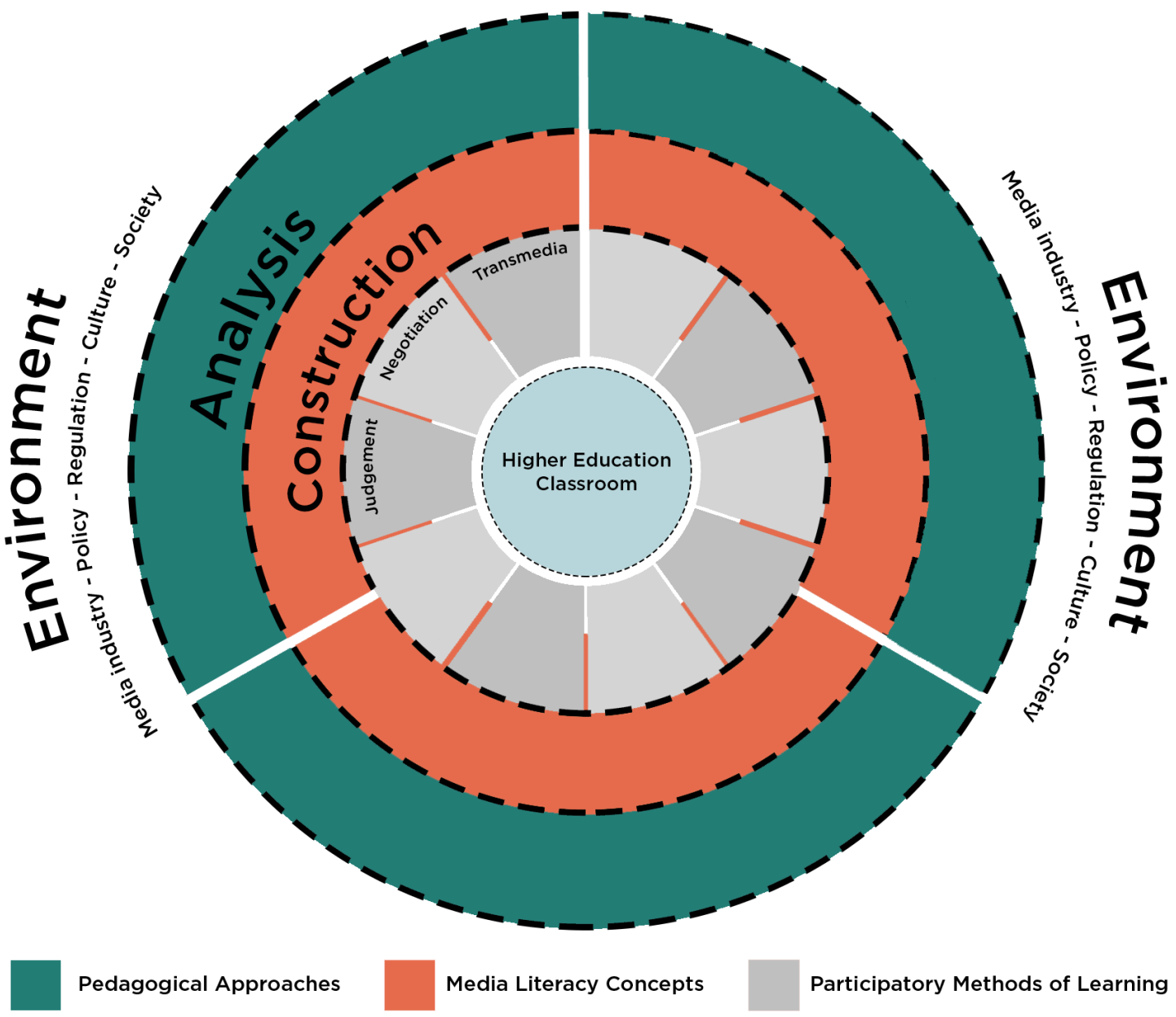

Note. This figure shows an example of the participatory ecological framework being used in a scenario that involves the pedagogical approach of analysis, the media literacy concept of construction, and the participatory learning methods of judgment, negotiation, and transmedia navigation.

First, using the judgment participatory learning method, the students and instructor analyze the credibility of some example messages from both candidates by discussing the social constructivism (Who made the choices that led to the creation of these messages?) and semiotics (What language was used in constructing these messages?) of the messages. Next, utilizing the negotiation participatory learning method, the discussion 
centers around the different perspectives that emerge in analyzing the messages from the different individuals within the classroom, based on the diverse communities they come from. Finally, the classroom activity concludes with an analysis of the transmedia navigation of both candidates' messages, and how they flow across different media platforms that include television commercials, print ads, and posts shared on various social media platforms; specifically looking at how language is used in differing ways across these different media channels.

Example scenario \#2. The second working example of the participatory ecological framework of media literacy takes place in a public four-year university in the midwestern U.S. The course, Digital Literacy, is an elective course in the teacher education program, intended for students who plan on teaching in elementary and secondary schools in any variety of subject areas. It is most commonly taken by students in their junior year, prior to any field experience courses.

The example takes place as the third of three class periods that utilize the framework; in the first two classes the education students worked through the pedagogical approaches of analysis and reflection regarding a social gaming application that is currently popular among middle and high school students in the U.S. The coursework has now moved on to the final lesson spotlighting production. In this class activity, the students focus on the core media literacy concept of positionality, utilizing the participatory methods of performance, appropriation, and visualization to create an interactive lesson plan that employs the gaming app as a learning tool for their future students (see Figure 10). 


\section{Figure 10}

\section{Example Scenario \#2}

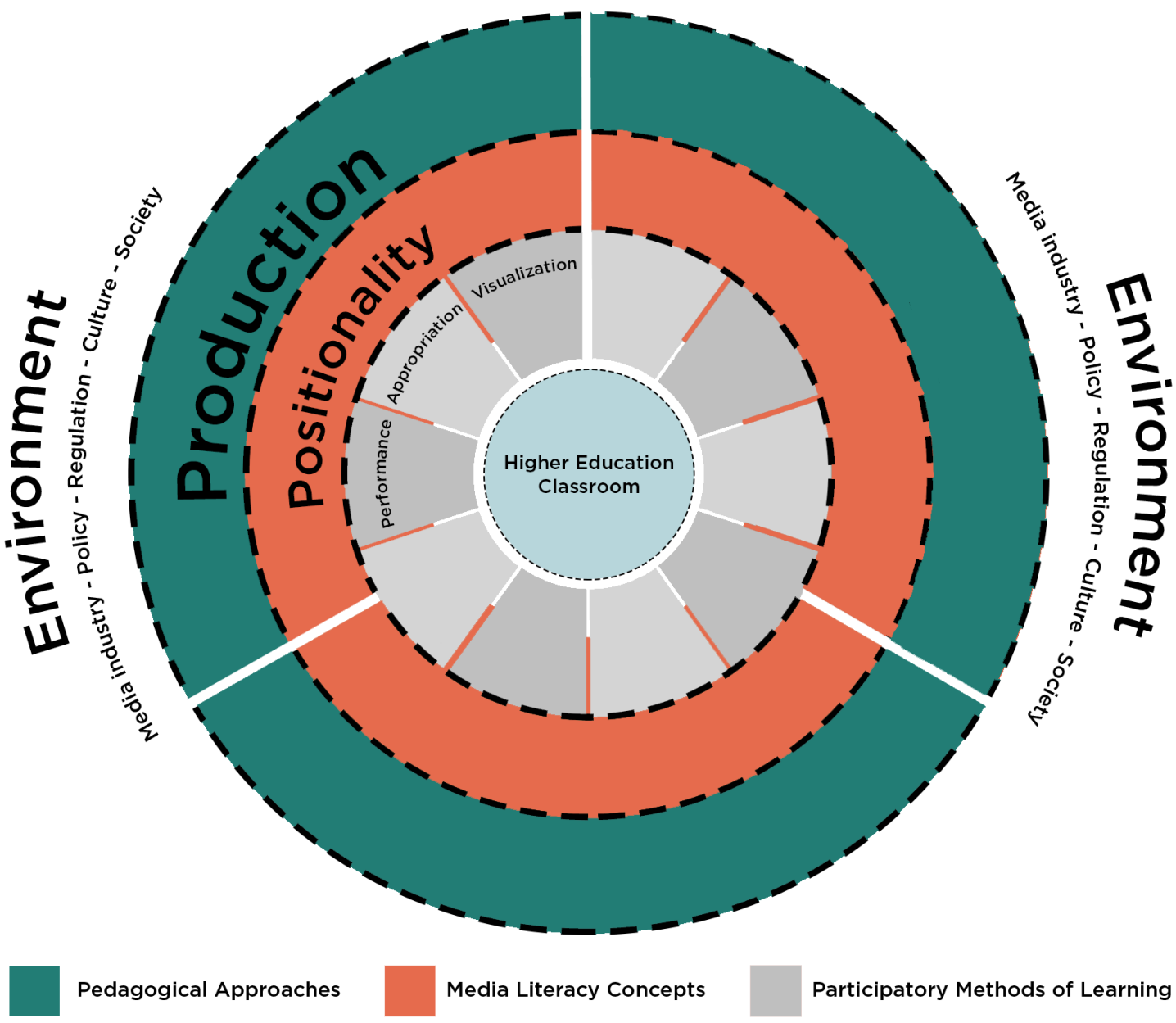

Note. This figure shows an example of the participatory ecological framework being used in a scenario that involves the pedagogical approach of production, the media literacy concept of positionality, and the participatory learning methods of performance, appropriation, and visualization.

The media literacy concept of positionality includes issues of message audience (Funk et al., 2016), authorship, impact, interpretations, and response (National Association of Media Literacy Education, 2007). The education students will use performance to take on a different identity, specifically that of their potential future students, in order to experience something new to them. In this example, the social gaming application is the 
new material; this will help them to understand the audience of the app as well as the lesson plan that is produced at the end of this activity. Appropriation will be used in the production of the lesson plan, as the education students are required to sample and remix content from the gaming app and its attached support documentation into their lesson plans.

Finally, visualization is accomplished through creating visual representations of the app in their lesson plans, the activities taking place during the lesson, and the intended learning outcomes. The students are required to pay close attention to how their future audience will interpret the visual information and respond to it in regard to the lesson plan. At the conclusion of this activity, the teacher education students will have created a new media product in the form of an interactive lesson plan that uses the social gaming app as a learning tool, specific to the environment in which they are teaching, namely the educational setting and subject area they plan to teach in the future.

Example scenario \#3. The final example of the participatory ecological framework of media literacy in action occurs at a private four-year liberal arts college in the northeast U.S.; this college deemphasizes general education and instead emphasizes a focus on students' academic discipline. The course in this example is a standalone media literacy course that is a theoretical prerequisite for the journalism program.

This example is a single class activity comprising the analysis and reflection of a collection of print stories regarding recent episodes of police shootings. The students will analyze and reflect on three stories from three different newspapers from across the country; all three stories are about a specific incident that occurred one week prior. In this les- 
son, the students will focus on the media literacy concept of substance, which is comprised of issues of politics of representation, social justice (Funk et al., 2016), content, context, and credibility (National Association of Media Literacy Education, 2007), all of which are appropriate to the subject of the news stories in question. The participatory learning methods of visualization, judgment, networking, and negotiation are used in this example activity (see Figure 11). 


\section{Figure 11}

\section{Example Scenario \#3}

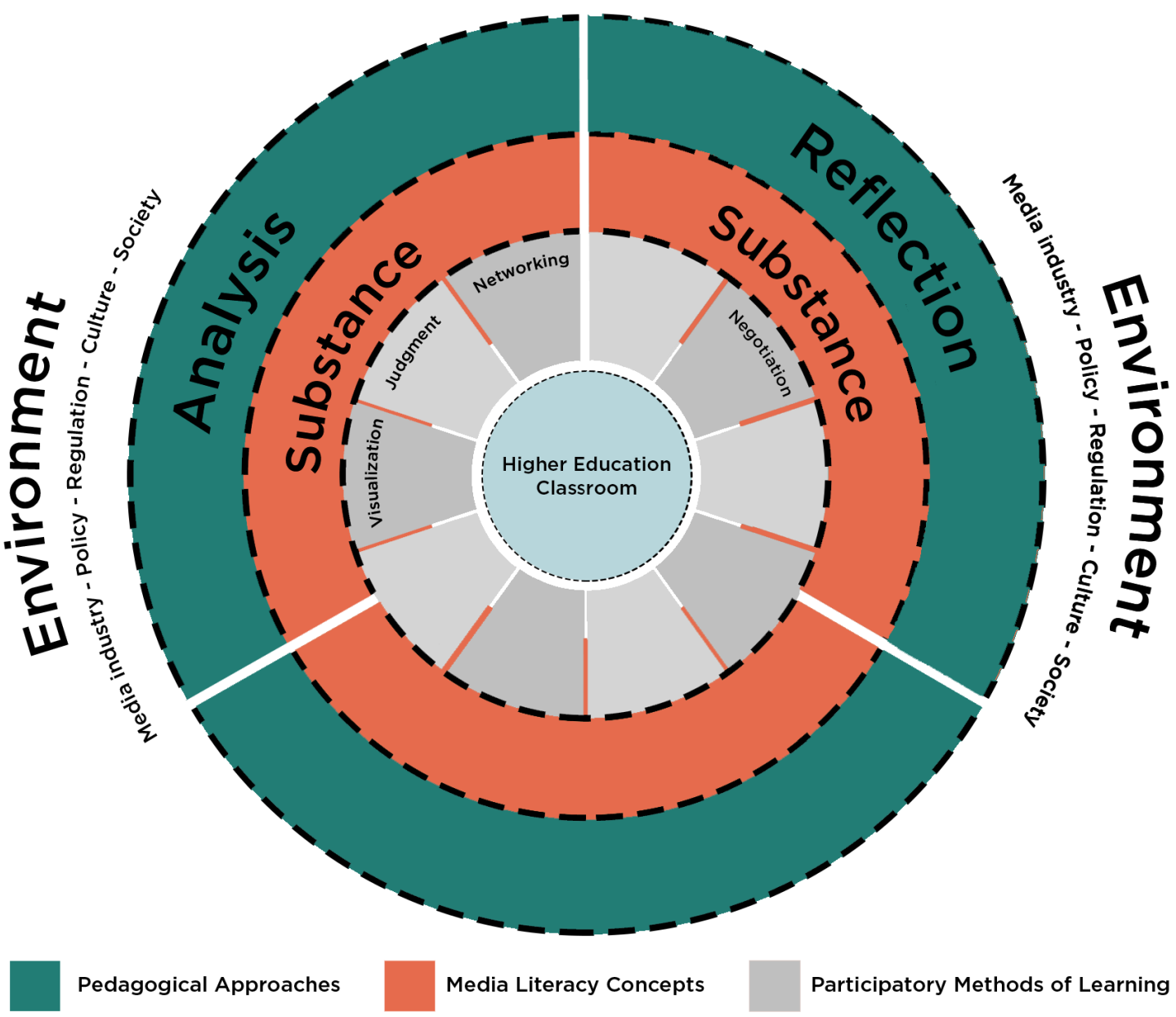

Note. This figure shows an example of the participatory ecological framework being used in a scenario that involves the pedagogical approaches of analysis and reflection, the media literacy concept of substance, and the participatory learning methods of visualization, judgment, networking, and negotiation.

The first pedagogical approach of an analysis of the substance of the three news stories is accomplished through the participatory learning methods of visualization, judgment, and networking. Visualization will take place as the students analyze the visual representations of images included in the news stories, specifically the accompanying photos and how they might be different across the three newspapers. Utilizing judgment, 
the students analyze the content and credibility of the stories, based on a perceived reliability of the sources cited in the three stories. Finally, the students use networking to search for elements of social justice and politics of representation and synthesize their findings from the three different stories; this information is later disseminated during the reflection portion of the lesson.

At the conclusion of the analysis phase of the lesson, the instructor and students in the classroom reflect on the similarities and differences found in the earlier analysis of the three media texts by utilizing the negotiation learning method. This participatory learning method focuses on how multiple perspectives can arise from diverse communities (Jenkins et al., 2009). By disseminating the information synthesized by the group in the analysis phase, they can then reflect on the similarities and differences found in the substance of the three texts by understanding how different perspectives might occur and be understood from different media sources covering the same story. This also gives the students the opportunity to understand how they as individuals understand the three stories in different ways, as well as how the external environment might influence any differences found among the stories.

\section{Conclusion}

Established through a synthesis of the scholarly review, this dissertation-in-practice proposes a new theoretical framework specific to the teaching of media literacy concepts. The participatory ecological framework of media literacy is based on Friere's (2010) viewpoint that transformative learning originates through critical dialogue, critical reflection, and action. Unlike many other empowerment frameworks for media literacy, 
the participatory ecological framework gives equal emphasis to the analysis, reflection, and creative production of media texts.

The framework allows faculty to take various pedagogical approaches to teaching media literacy through analysis, reflection, and production. The core media literacy concepts of positionality, construction, and substance are explored through participatory learning methods based on the work of Jenkins et al. (2009). The framework also takes into consideration the external environment, as well as the specific case of the individuals making up the classroom setting where instruction occurs. The framework is fluid in that the components of pedagogical approaches, media literacy concepts, and participatory learning methods can be used together in any possible combination particular to the setting and learning taking place. This framework can be utilized in any educational setting where media literacy is being taught, within any discipline, and does not require faculty to have expertise in traditional media studies.

\section{Summary}

Media literacy is still a nascent field of study when compared to many other academic disciplines, though the amount of progress and change that has taken place over the past two decades is noteworthy. This constant development mirrors the continual change in technology and media systems during the same period. It is known that the perception of a digital generation is a misinterpretation, in that inequalities exist in children and adults in regard to social and economic factors that define one's access, knowledge, and experience with both old and new forms of media (Buckingham, 2007). While there has been much development in the pedagogical frameworks of media literacy education during this period (Buckingham, 2003; Jenkins et al., 2009; Morrell et al., 
2013; Potter, 2004), frustration is still evident concerning the inclusion of media literacy in policymaking (De Abreu et al., 2014) and the inconsistencies concerning assessment tools and learning outcomes (Literat, 2014; Mihailidis, 2008).

Media literacy education is currently at an impasse. The literature has provided moderately paradoxical pedagogical frameworks through which it can be taught, but with a lack of policy backing in the U.S., discrepancies abound concerning the most effective pedagogical approach and the expected learning outcomes for media literacy in higher education (Mihailidis, 2008). While students are being exposed to this form of literacy through everyday interaction with the media outside of school, they are lacking in a fundamental understanding of media literacy that could be provided through proper classroom exposure and instruction (Buckingham, 2007). The introduction of the participatory ecological framework at the end of the scholarly review attempts to address the inconsistencies found in media literacy instruction in higher education settings. Moving forward, discussions of how media literacy instruction can be implemented in the current U.S. education system through policy development must be resolved for students to take full advantage of a critical understanding of this core cultural competency. 
Chapter Four: Contribution to Practice 


\section{Ensuring Successful Media Literacy Instruction in Higher Education: A Policy Brief}

\section{Key Points}

- Media literacy is an inquiry-based pedagogy that requires higher-level reasoning and critical thinking. This type of instruction is at odds with our current education system which emphasizes standardization and privatization.

- The pedagogical approaches and intended learning outcomes for teaching media literacy are inconsistent. This study shows that multiple, varying, and often conflicting frameworks are used by faculty in higher education settings.

- A lack of administrative oversight and policy support are environmental factors that contribute to discrepancies in media literacy instruction.

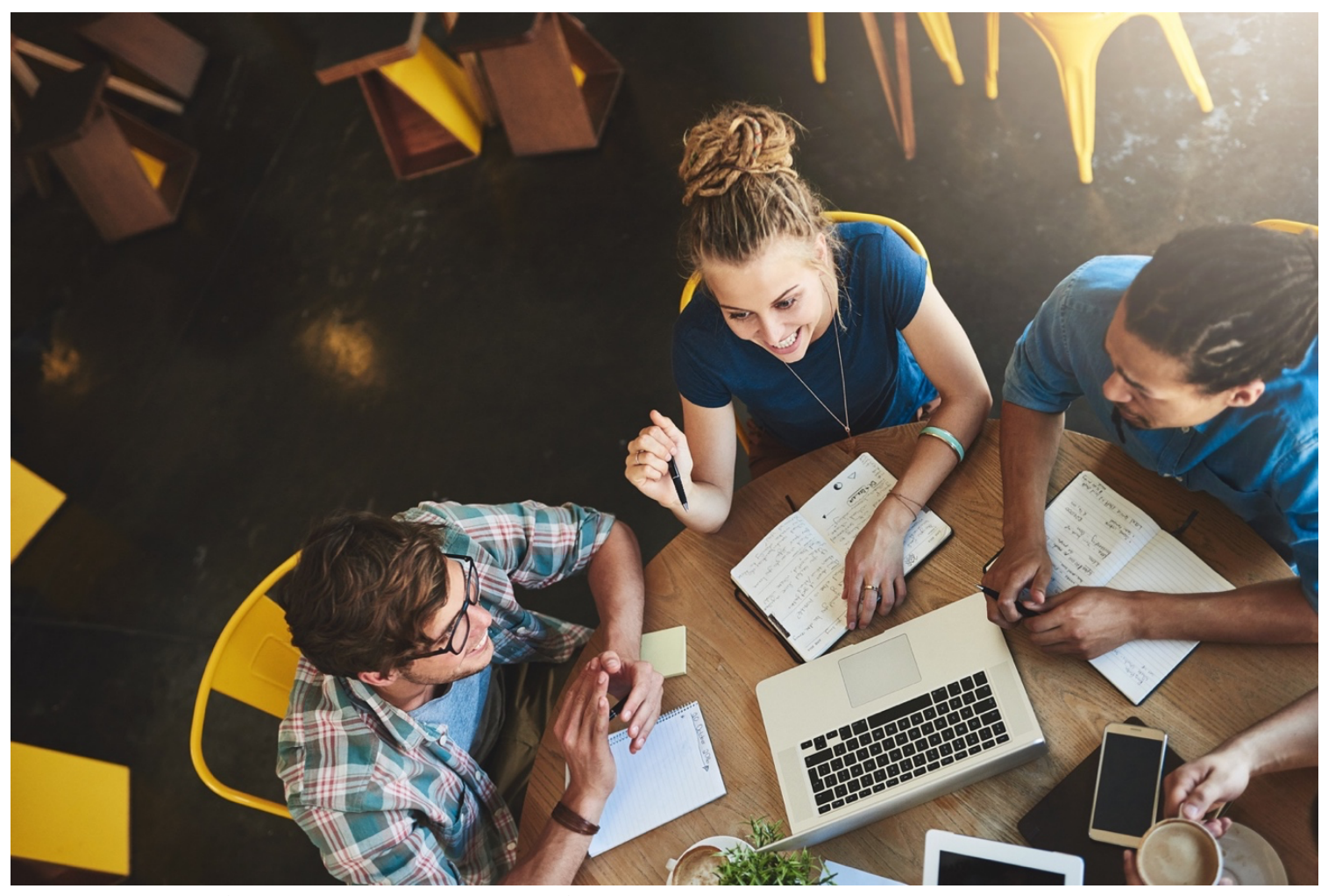

Photo courtesy of and used with permission from iStock (C)2020

\section{What's at Stake?}

We live today with an abundance of technological tools available at our fingertips at any given moment. The proliferation of high-speed Internet access, social media, mobile devices, and 
other digital technologies allow for exposure to media messages at ever increasing rates. With many Americans currently living in a reality of constant connectedness and information overload, the allocation of a scarce resource such as individual attention spans should be focused on relevant messages that produce value in our everyday experience.

Media literacy is the ability to access, analyze, evaluate, create, and act using all forms of communication'. The field is interdisciplinary by nature, representing a necessary, inevitable, and realistic response to the complex, ever-changing culture we live in. Media literacy also stresses critical thinking and creativity not only for the public to be critical consumers of media, but also to themselves become producers and communicators ${ }^{i i}$. A practical understanding of this form of literacy is a basis for effective knowledge production and active civic engagement in a democratic society.

Media literacy, while being an important issue in recent years, is still not a major field in the American education system and has little support or awareness from administrators ${ }^{\text {iii. }}$. A lack of policy support has contributed to discrepancies in the instruction and learning outcomes for media literacy in higher education, specifically. Inconsistencies in definition, use, and adoption have hindered media literacy's ability to produce tangible and coherent learning outcomes for university students ${ }^{\text {iv }}$.

The focus of media literacy instruction should be the use of media content to meet learning outcomes; however, there is a gap in the literature about what learning has actually taken place $^{4}$. Assessment tools for media literacy education are also scarce, and educators have emphasized the need for a national standard of media literacy assessment ${ }^{v}$. Finally, there is a lack of consistency in pedagogy, with competition taking place among the different conceptual models through which media literacy is taught.

To identify these inconsistencies, proposed here is a participatory pedagogical framework founded on critical thinking as a method for analysis, reflection, and creative production of media. This is accomplished through a qualitative study of 24 undergraduate courses that include elements of media literacy instruction, and a comparison of the findings from the qualitative study against the theoretical framework.

This brief aims is to inform university policy development by sharing the pedagogical approaches, intended learning outcomes, and environmental factors that influence the teaching of media literacy in higher education institutions in the U.S.

\section{A New Framework}

Media literacy instruction should take an ecological approach in which it is acknowledged that media and audiences interact together in a complex interrelated system of technologies and culture. Grounded in this viewpoint, this brief proposes and is guided by an empowerment framework for media literacy instruction that is applicable in all instructional settings.

This new framework, entitled the participatory ecological framework of media literacy, is grounded in both ecological and participatory approaches to pedagogy (see Figure 12). Based on 
an analysis of the empowerment frameworks found in the literature and a synthesis of their pertinent components, the framework included herein is founded on critical thinking as a method for analysis, reflection, and creative production of media.

The framework can be visualized by a series of three concentric circles, with varying components enclosed within each, surrounding a single entity of a classroom consisting of students and faculty.

Figure 12

Participatory Ecological Framework of Media Literacy

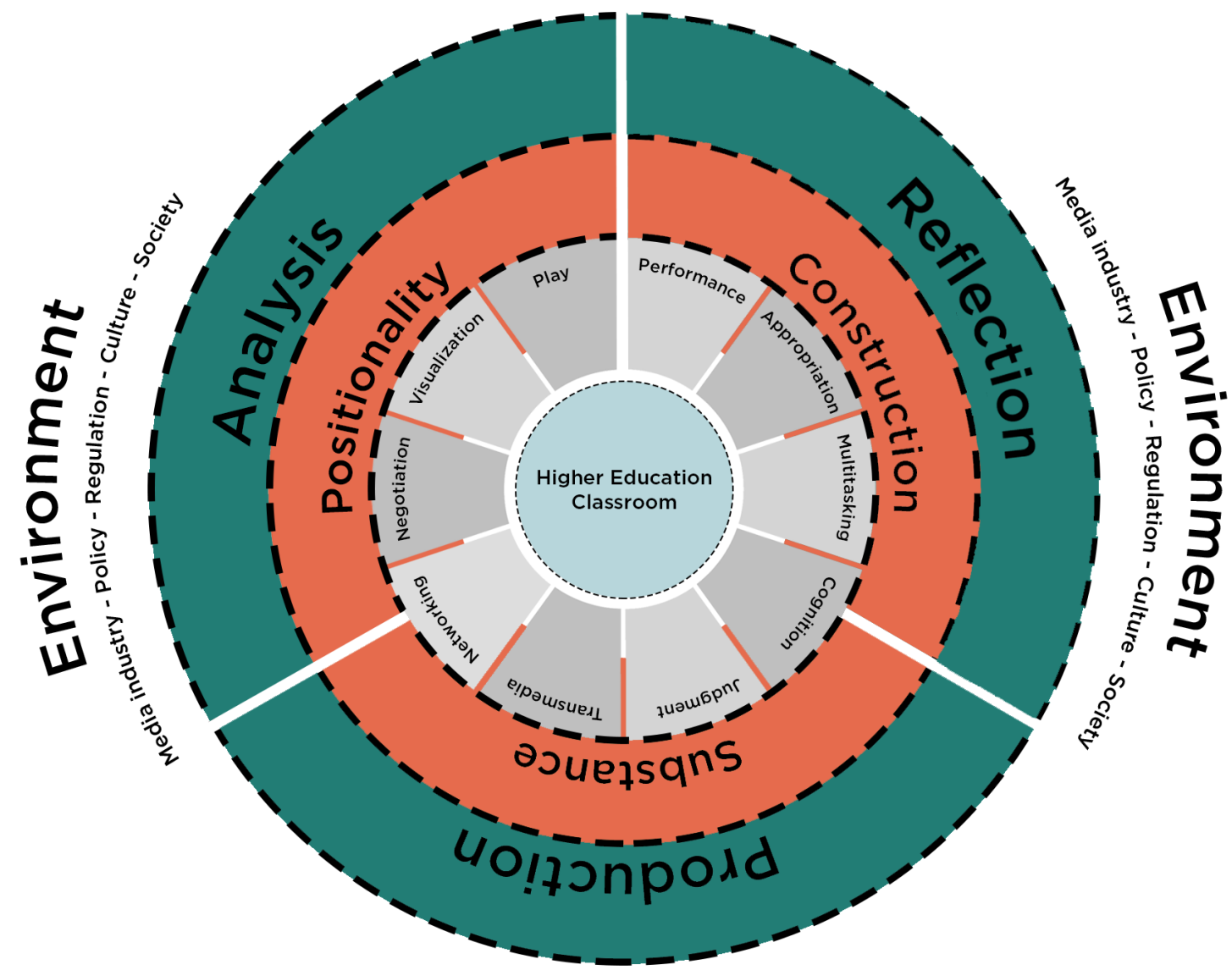

Pedagogical Approaches

Media Literacy Concepts

Participatory Methods of Learning

Note. This figure shows the components of the participatory ecological framework, including the pedagogical approaches, media literacy concepts, and participatory methods of learning.

This framework does not occur in isolation; instead it exists within the real-world environment that includes the media industry, policy, regulation, culture, and society. 
The outer circle contains the three pedagogical approaches of analysis, reflection, and production of media. The second circle consists of the media literacy concepts of positionality, construction, and substance. The third circle includes the ten participatory methods of learning: play, performance, appropriation, multitasking, distributed cognition, judgment, transmedia navigation, networking, negotiation, and visualization.

It should also be noted that the boundaries between these three circles, along with the boundaries separating the center and outside of the framework, are permeable, meaning that the environment as well as the individuals in the classroom will influence the learning taking place.

This framework allows teachers to merge the pedagogical approaches, media literacy concepts, and participatory methods of learning in any possible combination in their curriculum. This framework is used here to better understand the current landscape of media literacy courses being taught in higher education institutions in the U.S.

\section{Methods}

Data was collected from publicly available documents such as course syllabi, program overview statements, general education overview statements, and policy statements from 24 undergraduate-level courses that focused on, or included elements of, media literacy instruction, from 24 different institutions in the U.S. This included 12 courses from public universities, and 12 courses from private universities.

From these sites, a smaller subset was selected for follow-up interviews. This second phase of data collection consisted of semistructured, qualitative interviews with a subset of 6 faculty members from the sample courses in order to gain a deeper understanding concerning policies and administrative support for media literacy at their institutions.

The analysis presented in this brief was conducted using a qualitative design that included techniques of content and ethnographic analysis to focus on shared patterns across a sample of higher education courses in the U.S. containing elements of media literacy instruction.

Content analysis of documentary data addressed issues of how media literacy is being taught and what the intended learning outcomes are for its instruction. The ethnographic analysis addressed the environmental factors that influence the teaching of media literacy in university settings.

At the conclusion of these analyses, the findings from both were considered collectively and in contrast to the participatory ecological framework of media literacy introduced in this brief, to gain a full understanding of the teaching of media literacy in higher education institutions in the U.S. 


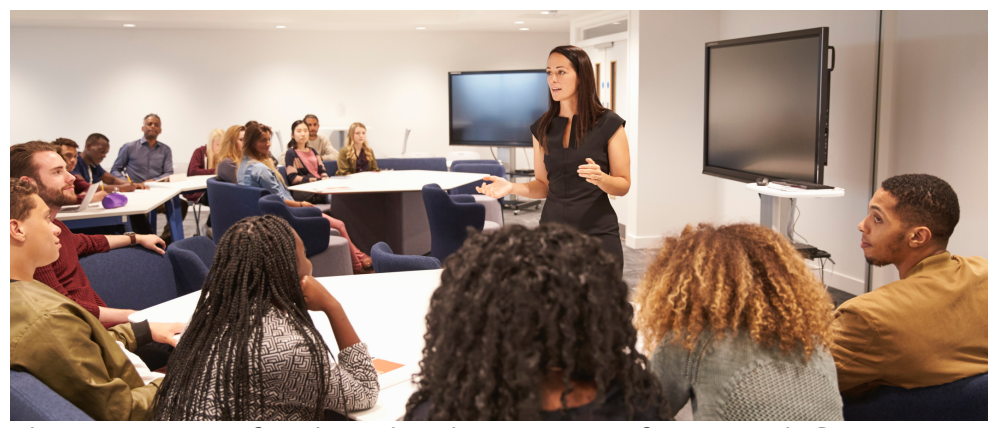

Photo courtesy of and used with permission from iStock (C)2020

\section{Results}

\section{Inconsistencies in Pedagogy \& Learning Outcomes}

Analysis of data revealed an unbalanced distribution of the pedagogical approaches of analysis, reflection, and production of media (see Table 2). Nearly all (92\%) of the courses taught at public universities included all three pedagogical approaches. In contrast, of the 12 private university courses analyzed in this brief, all 12 courses included elements of analysis, 10 of the courses included reflection, and media production was present in only 7 of the courses.

While the media literacy concepts of positionality, substance, and construction were present throughout all of the courses included in this brief, the participatory methods of learning were inconsistently applied across them.

Table 3

Findings on the Inclusion of Participatory Methods of Learning

Participatory methods of learning

$\begin{array}{cc}\text { Public } & \text { Private } \\ \text { university } & \text { university } \\ \text { courses } & \text { courses }\end{array}$

\begin{tabular}{|l|c|c|}
\hline Play & 8 & 7 \\
\hline Performance & 5 & 2 \\
\hline Appropriation & 4 & 3 \\
\hline Multitasking & 10 & 8 \\
\hline Distributed cognition & 11 & 7 \\
\hline Judgment & 7 & 6 \\
\hline Transmedia navigation & 3 & 4 \\
\hline Networking & 10 & 11 \\
\hline Negotiation & 8 & 9 \\
\hline Visualization & 7 & 5 \\
\hline
\end{tabular}


Analysis of the intended learning outcomes of media literacy courses reflect the findings of the pedagogical approaches, namely that the courses at public institutions combined both critical and creative approaches to learning more often than their counterparts at private institutions.

Examining the learning outcomes from all of the courses in this brief does reveal the breadth of lenses through which media literacy is currently being taught, supporting the belief that media literacy skills can be learned in any context. However, it was also revealed that faculty still do not fully grasp the critical thinking and inquiry-based learning methods required for a critical field such as media literacy.

\section{Environmental Factors that Influence the Teaching of Media Literacy}

Disparity of faculty capabilities and expertise

Lack of administrative direction

- Lack of institutional policy oversight

Faculty can be separated into two groups, defined in this brief as mavericks and passives.

Maverick faculty displayed innovation through the creation of multiple, unique media literacyfocused courses in varying disciplines, their teaching styles, the assignments included in their courses, and their willingness to push their students to think critically and expand their own media literacy knowledge. Mavericks also displayed an understanding of active learning concepts, encouraging their students to create products tailored to their particular learning styles, while allowing them to produce media texts based on their own learning.

In contrast, passives tended to be teaching their media literacy courses based on previous plans of study designed by other faculty. Most (75\%) of the passive instructors included in this brief were adjunct faculty. While these faculty expressed high interest in media literacy pedagogy, their syllabi and commentary displayed their teaching approach as viewing media literacy as a content area rather than a pedagogy that can be taught in any context. At no point did these faculty demonstrate a willingness to create innovative instruction that allowed for active learning to take place, rather the creation of a safe environment for students to better learn how to use technologies and recreate an expected product.

The principal environmental similarity between these two groups of faculty was the lack of administrative or policy oversight in how they teach their courses. Administrative involvement was negligible for all of these courses; both maverick faculty claimed to have created their courses of their own volition, with near-total autonomy at all stages of course design and implementation. 
The passive faculty noted little to no oversight for their media literacy courses as well, aside from the adjunct faculty being instructed to teach from the lesson plans already developed for their courses.

All of the faculty interviewed for this brief confirmed that there were no known policies at their institutions guiding the teaching of media literacy, whether those be institution-wide or departmentally focused, regardless of the discipline in which media literacy was part of the curriculum.

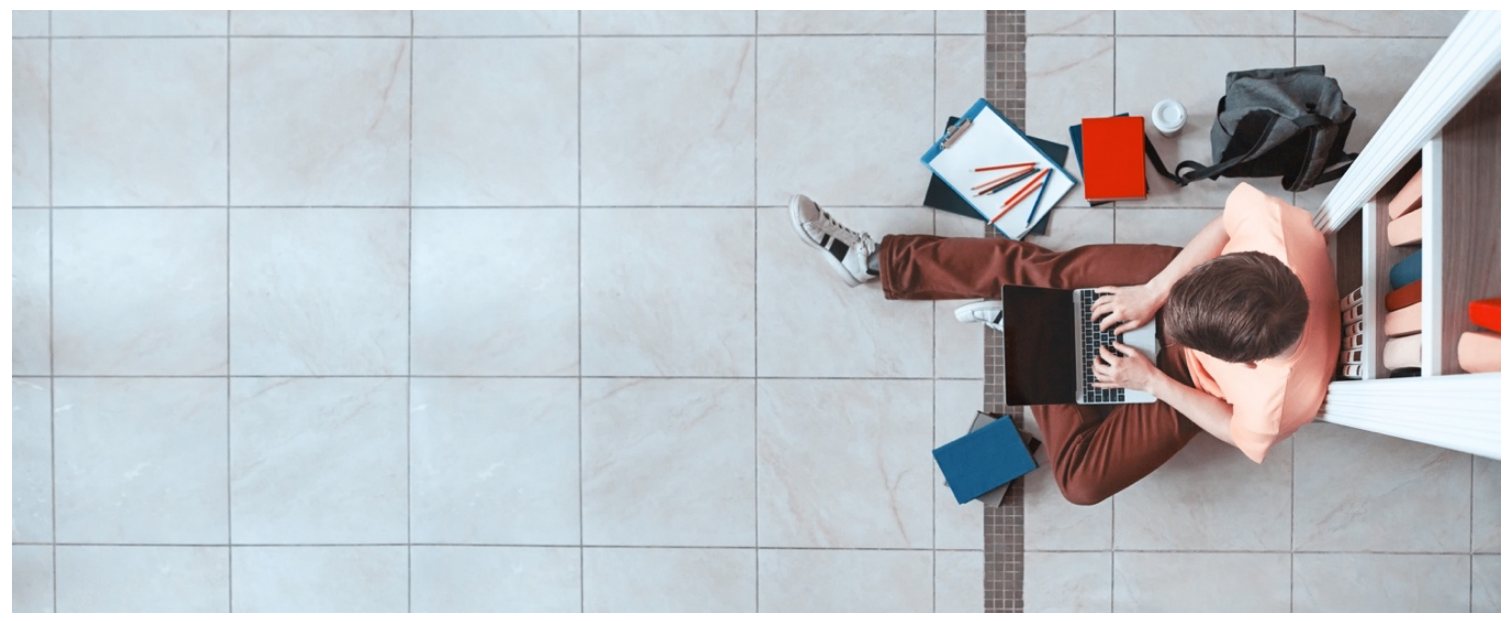

Photo courtesy of and used with permission from iStock (C)2020

\section{Recommendations}

- The inclusion of the pedagogical approaches of analysis, reflection, and creative production in all media literacy courses.

- Agreement among media literacy experts and faculty to utilize a single theoretical framework.

- Utilization of the participatory ecological framework of media literacy to ensure inclusion of the pedagogical approaches, media literacy concepts, and participatory methods of learning necessary for successful media literacy instruction.

- Administrative involvement and guidance in the design and development of media literacy courses.

Policy support at the institutional level to make clear the intentions and principles guiding media literacy instruction. 
Media literacy is a challenging subject to teach. If taught at a base level, students are asked to complete simple learning tasks, which is not significantly different than other subjects.

A small step to ensuring media literacy becomes the inquiry-based pedagogy it intends to be is the inclusion of the three pedagogical approaches of analysis, reflection, and creative production in all courses.

One solution to this problem would be the agreement of media literacy as a field to utilize a single theoretical framework, such as the participatory ecological framework of media literacy, as a starting point for all courses. This would ensure the inclusion of the necessary pedagogical approaches, media literacy concepts, and participatory methods of learning for all faculty teaching these courses.

It would be encouraged for faculty to still make their own decisions on how to utilize the framework in their courses, but this would at a minimum guarantee that all instruction has a uniform base level of pedagogical best practices.

Slight modifications to media literacy courses, or the incorporation of small, significant changes to current instruction would also be substantial. For example, by having opportunities for students to create their own questions and then produce something on a small scale would help media literacy embody the inquiry-based pedagogy it strives to be recognized as.

The inclusion of media literacy in higher education curriculum through institutional policy development is also recommended; administrative advocacy would make clear the intentions and principles guiding media literacy instruction at their institutions.

Issues such as consistency in pedagogy and the scope of its instruction at individual institutions could be substantiated through this policy inclusions. Additionally, the presence of media literacy in institutional policy can assist in future accreditation efforts as media literacy continues to become a more important, mainstream content area more akin to its level of prominence in other countries.

\footnotetext{
' Media literacy defined. (n.d.). National Association for Media Literacy Education. https://namle.net/publications/media-literacy-definitions

ii Buckingham, D. (2003). Media education: Literacy, learning and contemporary culture. Polity Press.

iii Kellner, D., \& Share, J. (2005). Toward critical media literacy: Core concepts, debates, organizations, and policy. Discourse: Studies in the Cultural Politics of Education, 26(3), 369-386.

iv Mihailidis, P. (2008). Are we speaking the same language? Assessing the state of media literacy in U.S. higher education. Studies in Media \& Information Literacy Education, 8(4), 1-14. https://doi.org/10.3138/sim.8.4.001 ${ }^{v}$ Literat, I. (2014). Measuring new media literacies: Towards the development of a comprehensive assessment tool. Journal of Media Literacy Education, 6(1), 2.
} 
MEDIA LITERACY IN HIGHER EDUCATION POLICY 195

Chapter Five: Contribution to Scholarship 


\author{
Media Literacy in Higher Education Policy: Pedagogical Choices \\ and Learning Outcomes
}

\begin{abstract}
This study proposes a participatory pedagogical framework founded on critical thinking as a method for analysis, reflection, and creative production of media through an understanding of the ways in which media literacy is being taught in higher education institutions in the United States. A qualitative design was utilized that included techniques of content and ethnographic analysis to focus on shared patterns across a sample of higher education courses in the U.S. containing elements of media literacy instruction. This study has found inconsistencies in the pedagogical approaches being utilized, a misunderstanding of the critical thinking and inquiry-based learning methods required for a critical field such as media literacy, and the lack of administrative oversight or policy support as key factors contributing to the discrepancies in media literacy's instruction and learning outcomes. Implications include recommendations for the field to consolidate theoretical frameworks, modifications to teaching, and policy and administrative advocacy efforts.
\end{abstract}

Keywords: media literacy, policy, pedagogy, learning outcomes, educational policy, higher education 
Media are a pervasive aspect of contemporary daily life and have a remarkable impact on our social discourse (Hobbs, 2010). As technological innovations allow for new forms of media, the public adapts to them, thereby changing the ways in which people interact with one another and seek out information. These perpetual changes in communication forms and technology mean that the act of learning how to successfully interact with media is a lifelong process. Combined with interest in the spread of misinformation and the popularization of colloquial terms such as "fake news," media literacy has recently shifted to become a fashionable topic in the eyes of the public. A critical understanding of media communication establishes a greater understanding of the processes and effects of media and is essential for effective knowledge production and civic engagement in a democratic society.

Despite the in-roads currently being made at the state level, media literacy education in the United States is still lacking a fully developed set of policies such as those found in Canada and countries in the European Union (Andersen, 2013). Although media literacy instruction in the U.S. is expanding with its inclusion in core competency standards in PK-12 education, a scarcity of policy support in higher education has contributed to discrepancies in definition, adoption, and instruction (Mihailidis, 2008). This lack of consistency manifests itself through a gap in the literature concerning media literacy pedagogy, assessment tools, and learning outcomes.

With no consensus present for the methods and contexts in which media literacy is currently being taught (Mihailidis, 2008), utilizing a framework founded on critical thinking is required for media literacy to successfully be taught across various educational contexts by faculty with differing levels of expertise. Faculty address support for 
the inclusion of media literacy in their programs of study, as well as the importance for institutional policies guiding its pedagogy and implementation within the curriculum. Further, the inconsistencies in understanding how media literacy should be viewed as a content area, the contexts in which it can be taught, and the lack of a determined theoretical framework across the subject matter area are hindering its understanding for students, other faculty, and the public (Hobbs, 2010; Mihailidis, 2008). This paper proposes a participatory pedagogical framework founded on critical thinking as a method for analysis, reflection, and creative production of media through an understanding of the ways in which media literacy is being taught in higher education institutions in the U.S. This framework guides the study and answer the following research questions:

1. How is media literacy being taught in higher education institutions in the U.S.?

2. What are the intended learning outcomes of media literacy instruction in higher education institutions in the U.S.?

3. What environmental factors influence the teaching of media literacy in higher education institutions in the U.S.?

Our society has reached a saturation point in the amount of information we are subjected to on a daily basis and is often rife with misinformation; no longer is any single piece or form of media communication seen as having distinctive significance (Potter, 2004). An overabundance of media messages creates difficulty in detecting opinion from fact, inaccuracy from validity, or devalued media messages from invaluable ones (Potter, 2004; Thoman \& Jolls, 2003). Decades ago, Masterman (1985) argued why an education in media was an urgent priority. Masterman (1985) included media saturation, media's 
strong societal influence, the manufacture and dissemination of information, and the infiltration of the media into our democratic processes as some of the most important reasons for students to gain media literacy competencies. A practical proficiency in media literacy helps future generations connect their media literacy competencies to emerging ethical situations that will inevitably appear as they navigate citizenship in participatory digital communities.

Media literacy instruction is at odds with our current education system based on neoliberal ideals emphasizing standardization and privatization. Unlike many other developed nations, the U.S. is currently lacking national policy support for media literacy education. For instance, in 2007 the European Commission mandated that all member states develop an assessment strategy for measuring the media literacy competency of its citizens (European Union, 2007), leading to the accumulation of substantial datasets and research coming out of the E.U. While there are many areas of innovation occurring in the U.S., they tend to still be at a grassroots level and local in scope. The inclusion of media literacy education in university policy will expand its support in the U.S. and ensure that appropriate pedagogy and learning outcomes are established.

\section{Review of Literature}

Media literacy is the viewpoint from which we access the media, critically evaluate its content, and create meanings from the images, words, and sounds in the messages we encounter (National Association for Media Literacy Education, n.d.). This form of literacy promotes social justice, an understanding of current technology and communication skills, and comprehensive global competencies. Media literacy education offers a 
framework for understanding and negotiating meanings through active inquiry, self-expression and critical thinking; this understanding helps to develop engaged, knowledgeable citizens who are acculturated to living in our media-saturated society through lifelong use of their skills and experiences (Hobbs, 2010; National Association for Media Literacy Education, 2007; Morrell, et al., 2013). Media literacy proficiencies must be established in formal educational settings to ensure active membership in our current participatory culture founded on cutting-edge technologies and innovative communication platforms (Hobbs 2010; Jenkins, et al., 2009). The facilitation of effective media literacy education is based on constructivist pedagogy, allowing learners to actively embrace critical thinking and creativity through the applied reflection of analysis, discussion, and engagement with the media (Hobbs, 2010; Literat, 2014). It is a critical pedagogy that leads to transformative learning and attached to its definition are challenging ramifications that must be addressed.

\section{Critical Media Pedagogy}

The teaching of media literacy in our multicultural society naturally brings to students' attention the inequities present in society based on power dynamics and class, race, and gender discrimination. Cultural and media studies have exhibited the pervasiveness of media in contemporary society and the need for media literacy to address issues of injustice, multiculturalism, and social differences (Kellner \& Share, 2005). Critical media literacy instruction empowers students to critically interpret information in multiple formats, to question the status quo, and to become change agents (Funk, et al., 2016). This critical media literacy pedagogy is influenced by Freire's (2010) critical pedagogy framework, and it recognizes the political nature of education through challenging the societal 
hierarchies present in media texts (Funk, et al., 2016; HeeYoung Kim, et al., 2016). A critical media pedagogy ties directly to the idea of what it means to be an active citizen in our current participatory culture and offers a practical approach to strengthening civic engagement by reaffirming the notion of democracy through empowerment (Funk, et al., 2016).

Critical media literacy instruction takes place in a collaborative environment that empowers both students and instructors to use their knowledge and skills to be active citizens who challenge societal injustices. For critical pedagogy to be successful, teachers must accept a bottom-up approach that allows for students to bring their own experiences, culture, and insight to the classroom (Kellner \& Share, 2005). This pedagogy prepares students to analyze media message conventions, criticize dominant ideologies and stereotypes, and interpret the meanings intrinsic to media texts (Kellner \& Share, 2005). True critical media literacy pedagogy legitimizes student perspectives to the greatest degree through the understanding of media texts as products of social production and its emphasis on self-expression and social activism (Kellner \& Share, 2005). It is also a transformative pedagogy through the utilization of a theoretical foundation combined with practical teaching methodologies that empower students to critically scrutinize dominant ideologies (Funk, et al., 2016).

In contrast to other instructional practices, critical pedagogy is not traditional in the sense that it uses well-established texts, assumptions, or teaching methods; instead, it takes a democratic approach to learning that involves teachers sharing classroom control with students as they work collaboratively to challenge authority. Funk et al. (2016) developed a set of six concepts and questions that can be used as a structural framework for 
this critical instruction, which include (a) social constructivism, (b) language and semiotics, (c) audience and positionality, (d) politics of representation, (e) production and institutions, and (f) social justice. Studies of instruction utilizing critical media literacy in PK12 settings emphasize that this pedagogy is most successful when students are empowered through active participation in the instruction (Gainer, 2010). The results affirm the importance of collaboration among instructors and learners as co-facilitators in the learning taking place through critical pedagogy. Ultimately, this framework situates learners as active change agents within their media literacy instruction by empowering them to address social injustices found in media through a critical interpretation of messages. This active approach to pedagogy is in line with post-secondary education's goal of fostering independent, lifelong learners.

\section{Media Literacy in Higher Education}

The inclusion of media literacy in university curriculum allows students to gain proficiencies such as critical thinking, an understanding of ethics and identity, connecting threads between media and civil society, and the ideals of engaged democracy (Mihailidis, 2008; Schwarz, et al., 2014). Higher education aims to create independent learners who continue to pursue knowledge in a process of ongoing, lifelong learning; gaining media literacy competencies while in college provides students with the ability to utilize their skills as they continue to seek relevant information throughout their lives. In opposition to this understanding, the education system in the U.S. traditionally has given little attention to media literacy (Heins \& Cho, 2003). This conflict arises when comparing the frameworks through which media literacy is taught, with a focus on group collaboration, creativity, active citizenship, and critical media production (Buckingham, 2009; Hobbs, 
2010; Morrell, et al., 2013), to current educational technology policy that aims for the efficient standardization of learning in order for students to gain proficiency in specific skills (De Abreu, 2014).

However, the subject has been making inroads in recent years, coinciding with the increased public prominence of media literacy, as educators realize this pedagogy is crucial to 21 st century learning (Jolls, et al., 2016). Media literacy's inclusion in curriculum in U.S. institutions of higher education is much less prevalent than in elementary or secondary education and can be seen as being nearly nonexistent in any kind of standardized fashion. A combination of limited research into the breadth of media literacy instruction in higher education settings and a lack of consistency in pedagogy and assessment for post-secondary curriculum is hindering many universities from formulating adequate policies and programs that address this challenge (Literat, 2014; Mihailidis, 2008). Compounding this issue is that while the basic definition of media literacy has been generally accepted for decades, the interpretation of that definition varies broadly among different academic disciplines (Mihailidis, 2008).

Regardless of an accepted belief in the need for media literacy proficiencies, few faculty members are qualified to teach the topic (Schwarz, et al., 2014). A study conducted by Schmidt (2012) found that faculty from varying disciplines identified the importance of students developing media literacy competencies, however most were unwilling to personally undertake this instruction due to systematic or personal factors. Similarly, many university students are unaware of media literacy's definition or existence (Schwarz, et al., 2014), with those not enrolled in mass communication or media studies 
majors unlikely to experience proper media literacy instruction during their university career (Duran, et al., 2008). Mihailidis (2008) identified inconsistent definitions, a focus of media literacy scholarship on PK-12 teaching, and the lack of adoption in the U.S. compared to many other countries as being the main reasons why media literacy curriculum is difficult to pinpoint in higher education. Because of the inequality of adoption when compared to PK-12 settings, identifying any type of standardized learning outcomes in the university is difficult (Mihailidis, 2008).

Assessment of accepted media literacy instruction outcomes has also largely been overlooked in higher education (Schilder, et al., 2016). Further, due to its inherent nature, higher education has a scarcity of national standards similar to those enacted by the National Communication Association or the current Common Core standards. The overall view of media literacy in higher education is one of duplicity; as new media and technology initiatives appear at ever-increasing rates, the ignorance and resistance regarding the subject persists, despite opposing views to its importance.

Due to these disparities and the absence of national policy regarding media literacy instruction in higher education settings, this paper aims to clarify the ways in which media literacy is being taught, the intended learning outcomes, and the environmental factors influencing the teaching of media literacy in higher education institutions in the U.S. After presenting these findings, a participatory pedagogical framework founded upon critical thinking as a method for analysis, reflection, and creative production of media is introduced, which is applicable to any discipline within university curriculum as well as in other educational settings such as PK-12 classrooms. 


\section{A Pedagogical Framework for Media Literacy}

As suggested by Jenkins et al. (2009), media literacy instruction should take an ecological approach in which it is acknowledged that media and audiences interact together in a complex interrelated system of technologies and culture. Grounded in this viewpoint, this study proposes and is guided by an empowerment framework for media literacy instruction that is applicable in all instructional settings. This new framework, entitled the participatory ecological framework of media literacy, is grounded in ecological and participatory approaches to pedagogy. Based on an analysis of the empowerment frameworks found in the literature and a synthesis of their pertinent components, the framework included herein is founded on critical thinking as a method for analysis, reflection, and creative production of media. The framework recognizes the definition of media literacy as being able to access media, understand and critically evaluate the different aspects contained therein, and being able to produce communications in a variety of contexts (European Union, 2007), while simultaneously being suitable for the teaching of media literacy at any level of education.

The participatory ecological framework of media literacy also takes a philosophical approach to pedagogy based on the work of Freire (2010). In this framework, Freire's (2010) guiding principles of critical dialogue, critical reflection, and action constituting a transformative pedagogy have been reconstructed into the three pedagogical approaches of analysis, reflection, and creative production of media. The framework can be visualized by a series of three concentric circles, with varying components enclosed within each, surrounding a single entity of a classroom consisting of students and faculty (see Figure 13). This framework does not occur in isolation; instead it exists within the real- 
world environment that includes the media industry, policy, regulation, culture, and society.

The outer circle contains the three pedagogical approaches of analysis, reflection, and production of media. The second circle consists of the media literacy concepts of positionality, construction, and substance. The third circle includes the ten participatory methods of learning as identified by Jenkins et al. (2009) and independently verified by Literat (2014); these are comprised of play, performance, appropriation, multitasking, distributed cognition, judgment, transmedia navigation, networking, negotiation, and visualization. It should also be noted that the boundaries between these three circles, along with the boundaries separating the center and outside of the framework, are permeable, meaning that the environment as well as the individuals in the classroom will influence the learning taking place. 


\section{Figure 13}

Participatory Ecological Framework of Media Literacy

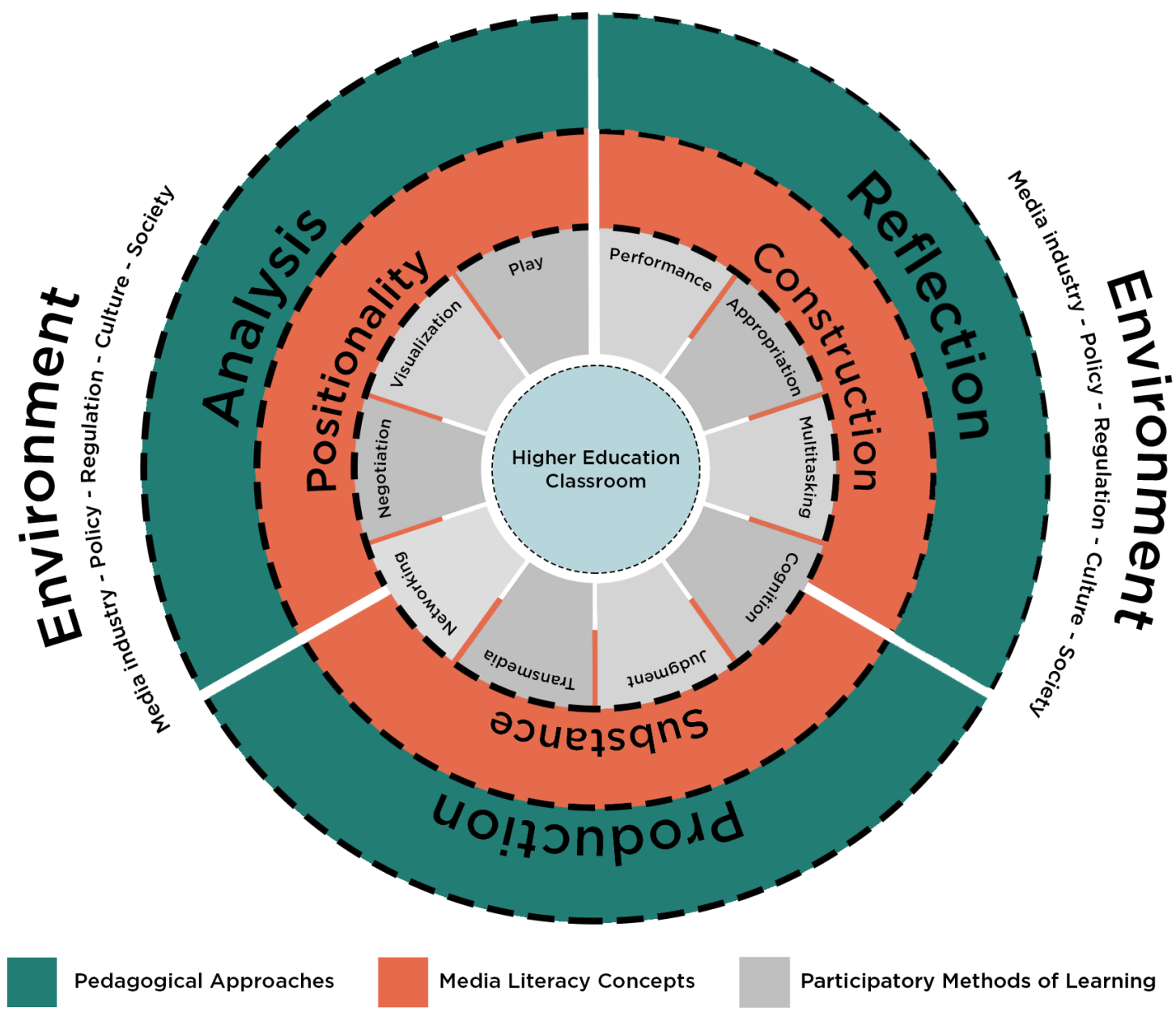

Note. This figure shows the components of the participatory ecological framework, including the pedagogical approaches, media literacy concepts, and participatory methods of learning.

The framework allows for teachers to merge the three pedagogical approaches in any possible combination in their curriculum. The pedagogical approaches of this framework are viewed as being of equal importance and are not sufficient in teaching media literary concepts without complementing one another. While a single pedagogical approach could be employed in a segment of a larger, more comprehensive lesson, the ulti- 
mate intention of utilizing the participatory ecological framework is to consistently employ all three pedagogical approaches equitably. Again, while these three approaches are based on Friere's (2010) principles of critical dialogue, critical reflection, and action, they should not be viewed as a sequence of steps to transformative learning; rather they are independent approaches that can be taught collectively as needed based on the intentions of the learning outcomes.

The second concentric circle contains the three core media literacy concepts of positionality, construction, and substance. Positionality includes media literacy components such as audience (Funk, et al., 2016), authorship, impact, interpretations, and response (National Association for Media Literacy Education, 2007). Construction consists of production, social constructivism, language and semiotics (Funk, et al., 2016), purpose, and techniques (National Association for Media Literacy Education, 2007). The final media literacy concept of substance includes issues of politics of representation, social justice (Funk, et al., 2016), content, context, and credibility (National Association for Media Literacy Education, 2007). The three media literacy concepts in this framework can work independently of one another or in any combination, and also separately or together through any of the three pedagogical approaches.

The third concentric circle includes the ten participatory methods of learning as identified by Jenkins et al. (2009) and confirmed through research conducted by Literat (2014). Specifically, these learning methods include play, performance, appropriation, multitasking, distributed cognition, judgment, transmedia navigation, networking, negotiation, and visualization. Similar to the media literacy concepts included in this framework, the participatory methods of learning can be used autonomously or collectively in 
any combination with all three pedagogical approaches, as well as include any of the three core media literacy concepts. This framework was used to examine course syllabi and accompanying documents to answer the research questions concerning pedagogical approaches and learning outcomes in media literacy courses being taught in higher education institutions in the U.S.

\section{Methods}

\section{Research Design}

This study utilized a qualitative design that included techniques of content and ethnographic analysis to focus on shared patterns across a sample of higher education courses in the U.S. containing elements of media literacy instruction. Content analysis is an inductive research approach and was selected to determine the presence of particular themes or words within the texts collected from the sample university courses (Merriam, 2009). Ethnographic analysis in the form of qualitative interviews was conducted in order to discover a deeper understanding of how institutional policies and culture possibly influenced the teaching of media literacy in the participant settings (Merriam, 2009).

The inclusion of 24 total data collection sites was intended to better the generalizability of the results (Creswell, 2014). This focused study design is well suited to investigating a practical problem such as approaches to media literacy education and the implications for its future policy development (Sykes, et al., 2009; Merriam, 2009). The content analysis of the documentary data addressed the research questions of how media literacy is being taught, and what the intended learning outcomes are for media literacy instruction in higher education institutions in the U.S. The ethnographic analysis addressed 
the third research question of what environmental factors influence the teaching of media literacy in higher education institutions in the U.S.

\section{Data Collection}

The first set of data collected was publicly available written documents consisting of course syllabi, program overview statements, general education overview statements, and policy statements from 24 undergraduate-level courses that focused on, or included elements of, media literacy instruction, from 24 different institutions in the U.S. This purposeful sampling method was selected with an underlying goal of discovery and verification of the pedagogical approaches and intended learning outcomes of post-secondary media literacy instruction (Altheide, 1987). Additionally, this substantial sample size should assist with ensuring as much variability as possible among the institutional settings.

Data was collected equally from 12 private and 12 public universities to better understand any possible differences in either setting (see Table 3). Data was collected from these 24 sites based on the following categories: (a) mass media and communication studies theoretical prerequisite courses, (b) general education courses, (c) teacher education courses, and (d) courses in various subject areas that integrate elements of media literacy education into the course content. Data was collected from three private four-year universities and three public four-year universities within each of these categories, thus equaling 24 total sites for data collection. 


\section{Table 4}

Criteria for Participant Data Collection Sites

\begin{tabular}{lllll}
\hline & $\begin{array}{l}\text { Mass media } \\
\text { and communi- } \\
\text { cation theoreti- } \\
\text { cal prerequisite } \\
\text { courses }\end{array}$ & $\begin{array}{l}\text { General educa- } \\
\text { tion courses }\end{array}$ & $\begin{array}{l}\text { Teacher educa- } \\
\text { tion courses }\end{array}$ & $\begin{array}{l}\text { Integrated } \\
\text { courses within } \\
\text { various other } \\
\text { subject areas }\end{array}$ \\
\hline $\begin{array}{l}\text { Public 4-year } \\
\text { institutions } \\
\begin{array}{l}\text { Private 4-year } \\
\text { institutions }\end{array}\end{array}$ & 3 courses & 3 courses & 3 courses & 3 courses \\
\hline
\end{tabular}

This allowance for maximum variability within the sample data enhances the transferability of this qualitative study (Merriam, 2009). The sampling of participant sites in this study can be seen as both convenient, due to the sample size not including the entire population of possible higher education courses in the U.S. containing elements of media literacy instruction (Krippendorff, 2004), as well as purposeful, since the sampling of participant sites are based on specific criteria (Merriam, 2009).

The media literacy courses included in this study were collected through Internet search engine queries that identified participant settings through publicly available syllabi that focused on, or included elements of, media literacy instruction. Identifying publicly available syllabi that met the requirements of this study, namely that a course does include elements of media literacy instruction, were subjective in nature and relied on the expertise of the researcher to correctly identify course syllabi that could be incorporated into the data sample. In order to allow for as wide a range of samples as possible, there was no expectation that the courses were traditional seated, hybrid, or online courses; data was collected regardless of the instructional design of the course. Additionally, no restrictions were placed on course length; for example, traditional 16-week semester 
courses, shorter intensive summer courses, or any other course length were considered valid with the understanding that the course is offered to students for academic credit. Once the sample courses were identified, appropriate accompanying documentation such as program overview statements, general education overview statements, and policy statements were also added to the data sample.

From these 24 participant sites, a smaller subset was selected for follow-up interviews at the conclusion of the collection and analysis of the documentary data. This second phase of data collection consisted of semi structured, qualitative interviews with a subset of 6 faculty members (see Table 5) from the sample courses in order to gain a deeper understanding concerning policies and administrative support for media literacy at their institutions.

\section{Table 5}

Faculty Participant Demographics

\begin{tabular}{lllll}
\hline & $\begin{array}{l}\text { Public 4-Year } \\
\text { Institutions }\end{array}$ & $\begin{array}{l}\text { Private 4-Year } \\
\text { Institutions }\end{array}$ & $\mathrm{n}$ & $(\%)$ \\
& & & & \\
\hline $\begin{array}{l}\text { Sex } \\
\text { Male }\end{array}$ & 0 & 1 & 1 & $17 \%$ \\
$\quad$ Female & 2 & 3 & 5 & $83 \%$ \\
Race/Ethnicity & 0 & & & \\
$\quad$ African American & 1 & 0 & 0 & $0 \%$ \\
White & 1 & 4 & 1 & $83 \%$ \\
Hispanic & 0 & 0 & 0 & $0 \%$ \\
Other & 1 & 0 & 2 & $33 \%$ \\
Years Teaching & 0 & 1 & 1 & $17 \%$ \\
$0-3$ & 0 & 1 & 0 & $0 \%$ \\
$3-5$ & 0 & 0 & 0 & $0 \%$ \\
$5-10$ & 1 & 0 & 3 & $50 \%$ \\
$10-15$ & & 2 & & \\
$15+$ & & & & \\
\hline
\end{tabular}


The purpose of these interviews was to gain a deeper understanding of how issues such as politics, policy, institutional support, and internal culture influenced the teaching of media literacy at their institutions (Merriam, 2009). Interview questions asked the participants for their perspectives on the creation or background of their course, the theories or frameworks utilized, their personal experiences teaching the course, as well as the effects of any environmental factors or polices present at their institutions.

The interviews were semi structured, ranged in time from 25 to 60 minutes, and a full recording and transcription of all conversations between the researcher and interviewees was conducted for further analysis. The original intended number of interviews in the second phase of data collection was 8 faculty, representing one interview from each category of data collection; however, due to unforeseen availability and contact issues, only 6 interviews were conducted. The collection of ethnographic data allowed for further description and interpretation of issues outside of the pedagogical approaches and learning outcomes that could not be fully understood solely from content analysis of the documentary data (Merriam, 2009).

\section{Data Analysis}

The first step of data analysis, which examined the written documentation data of course syllabi, program overview statements, general education overview statements, and policy statements, aimed to determine the presence of particular themes or words within the data in order to find generalizations in the pedagogical approaches and learning outcomes in the data. The coding rubric utilized during analysis was based on the participatory ecological framework of media literacy introduced earlier in this study. The compo- 
nents of the coding rubric included the three pedagogical approaches of analysis, reflection, and production of media; the media literacy concepts of positionality, construction, and substance; and the ten participatory methods of learning as identified by Jenkins et al. (2009) and independently verified by Literat (2014): play, performance, appropriation, multitasking, distributed cognition, judgment, transmedia navigation, networking, negotiation, and visualization. The analysis of the written documentary data aimed to clarify the ways in which media literacy is being taught and the intended learning outcomes for post-secondary media literacy courses.

After analyzing the documentary data, follow-up interviews took place with faculty members who taught 6 of the 24 sample courses. The coding approach used for analysis of the interview data was a combination of the same classification schema used for analyzing the documentary data as well as additional categories that emerged during the interviews (Merriam, 2009). This extended classification system for the ethnographic analysis allowed for sociocultural data to emerge that would otherwise go unnoticed from the content analysis phase of the study. Once the analysis of the interview data was completed, the findings from both the documentary content analysis and the ethnographic interview analysis were considered collectively in order to identify the environmental factors influencing the teaching of media literacy in higher education institutions in the U.S.

\section{Results}

The researcher conducted separate sets of analyses of the documentary and interview data, then combined those findings to answer the three research questions of how 
media literacy is currently being taught, the intended learning outcomes, and the environmental factors that influence the teaching of media literacy in higher education institutions in the U.S. The findings for each research question are listed separately below.

\section{Research Question 1}

The first research question sought to understand how media literacy is being taught in higher education institutions in the U.S. The documentary analysis of syllabi and related text materials from the 24 university courses in this study revealed an unbalanced distribution between public and private settings in regard to the pedagogical approaches of analysis, reflection, and production of media. The majority (92\%) of the 12 public university courses included all three pedagogical approaches; 11 of the courses included analysis and reflection, while all 12 included media production. In contrast, of the 12 private university courses, all 12 courses included elements of analysis, 10 of the courses included reflection, and media production was present in only 7 of the courses. Next, the researcher identified that the media literacy concepts of positionality, construction, and substance were present in all 24 courses included in this study.

The final component of the coding rubric was a search of the ten participatory methods of learning as identified by Jenkins et al. (2009) and verified by Literat (2014). Similar to identifying pedagogical approaches and media literacy concepts, the participatory methods of learning were identified by the researcher through hand-coding course syllabi from the participant sites. For example, one syllabus stated that students would "take on the role of an academic imagineer," in reference to the course being taught through the lens of Disney. This indicated the inclusion of the participatory method of 
learning of performance, which is defined as adopting alternative identities for the purpose of discovery and improvisation (Jenkins et al., 2009). Another example was the inclusion of negotiation, which involves respecting multiple perspectives from a diverse number of communities (Jenkins et al., 2009). One course syllabus emphasized community-based learning, with students creating and presenting their capstone projects to multiple diverse audiences within their local communities; many of the assignments in this course also included this participatory method of learning which culminated in the presentation of their final projects. Table 6 provides a complete list of the participatory methods of learning for both public and private university courses. The 6 follow-up interviews with faculty confirmed the findings in this section for their particular courses.

\section{Table 6}

Findings on the Inclusion of Participatory Methods of Learning

\begin{tabular}{lll}
\hline $\begin{array}{l}\text { Participatory methods of } \\
\text { learning }\end{array}$ & Public university courses & Private university courses \\
& & \\
\hline Play & 8 & 7 \\
Performance & 5 & 2 \\
Appropriation & 4 & 3 \\
Multitasking & 10 & 8 \\
Distributed cognition & 11 & 7 \\
Judgment & 7 & 6 \\
Transmedia navigation & 3 & 4 \\
Networking & 10 & 11 \\
Negotiation & 8 & 9 \\
Visualization & 7 & 5 \\
\hline
\end{tabular}

\section{Research Question 2}

The findings for the second research question investigating the intended learning outcomes of media literacy courses reflect the findings of the pedagogical approaches in RQ1, namely that the courses at public institutions combined both critical and creative 
approaches to learning more often than their counterparts at private institutions. However, examining the learning outcomes from all of the courses in this study reveals the breadth of lenses through which media literacy is currently being taught, supporting the belief that media literacy skills can be learned in any context. Learning outcome themes that emerged from the mass media prerequisite courses included an equal emphasis for the three pedagogical approaches of analysis, reflection, and production. Participatory learning methods were also given priority in these courses, with project-based learning, group projects, and active learning being present throughout, incorporating not only production but also critical thinking outcomes tied to analysis and reflection. These mass media courses reiterated concepts of digital citizenship and its link with media literacy; issues of social change, symbiotics, hegemony, representation, empowerment, and community and social change were themes that appeared in these mass media courses.

The learning outcomes in the general education courses were more fundamental in nature compared to the mass media prerequisites, with substantial emphasis on analysis of media texts. Since these general education courses are intended for students in any discipline, the learning outcomes skewed towards an understanding of basic media literacy theoretical frameworks with little significance given to demonstrating an active understanding of creative media production or critical reflection. Demonstration of production knowledge as a learning outcome was present in 2 general education courses, while critical reflection was only present in a single course. A final theme that emerged from the general education courses was a focus on concepts of digital literacy in lieu of media literacy; issues concerning digital technologies and their use were prevalent in these courses. 
Media literacy courses within teacher education programs also de-emphasized the pedagogical approach of production. Gaining a theoretical understanding of media literacy concepts and then demonstrating how one would incorporate that knowledge into teaching materials such as lesson plans was the central theme in all 6 of the teacher education courses in this study. Similar to the general education courses, digital literacy and technology topics were often emphasized over, or confused with, concepts related to media literacy. Protectionist ideology was also common, with a focus on the current media landscape, the role of media, and how media affects youth being present in multiple courses.

Finally, the learning outcomes from media literacy courses found within various other disciplines varied widely, corresponding with the subject area where they were found. For instance, a music course focused almost solely on production, while journalism, child psychology, and religious studies courses only included the pedagogical approach of analysis of media texts pertinent to their discipline. Two of the 6 courses did, however, include all three pedagogical approaches in their learning outcomes; beginning with a theoretical understanding of media literacy concepts, both courses progressed through assignments that ultimately led to students demonstrating their understanding through critical reflection and production projects that were also tied closely to their discipline. Again, similar to RQ1, the faculty interviews were utilized to confirm the findings from RQ2.

\section{Research Question 3}

The third research question in this study sought to discover the environmental factors that influence the teaching of media literacy in higher education institutions in the 
U.S. Two central themes emerged from the qualitative interviews conducted with faculty teaching the courses included in this study. First, the faculty members can be separated into two groups, labeled here as mavericks and passives. The term maverick is synonymous with one who has the leeway to create curriculum and teach it in any manner they see fit; passives refer to faculty who teach the material at a base level, with little to no input or creativity of their own injected into the pedagogical approach of their courses. Of the 6 faculty interviewed in this study, 2 faculty members would be considered mavericks, while the other 4 instructors would fall into the passives group. The second theme to emerge from the findings in regard to the environmental factors influencing the teaching of media literacy is the amount of administrative and policy oversight given to media literacy as a pedagogy or to the teaching of media literacy concepts at these institutions is noticeably slight, if not completely absent.

The maverick faculty displayed innovation through the creation of multiple, unique media literacy-focused courses in varying disciplines, their teaching styles, the assignments included in their courses, and their willingness to push their students to think critically and expand their own media literacy knowledge. One of these maverick faculty created her course on a dare from a colleague. She stated, "we're allowed to teach special topics classes. He dared me to teach a class about Disney; I told him, well, it's going to be about pedagogy because that's what media literacy is about." These faculty view media literacy as a critical pedagogy, with one saying, "you need to teach them how to think, it's all about pedagogy." The other faculty member echoed this approach. "It's not teaching people what to think. It's teaching them how to discern information to then know how to make ... those choices." 
Both of the instructors who would be considered mavericks displayed an understanding of active learning concepts, encouraging their students to create products tailored to their particular learning styles, and allowing them to produce media texts based on their own learning. The pedagogical approaches of analysis, reflection, and production were found equally in all of the courses taught by these faculty, with the ten participatory methods of learning as identified in the literature review also present at varying degrees in their courses. The courses taught by these mavericks included mass media prerequisite courses and courses falling within other disciplines outside of media literacy or mass media studies.

The instructors labeled here as passives tended to be teaching their media literacy courses based on previous plans of study designed by other faculty; 3 of the 4 instructors falling within this group were adjunct faculty. One of the adjuncts stated, "I teach what they give me. I don't have very much leeway to change the syllabus." The pedagogical approaches of analysis, reflection, and production were unbalanced, with analysis being much more prevalent. These courses included mass media prerequisites, general education courses, and teacher education courses. The passive faculty teaching in the area of teacher education shared, "my course is all about identifying the best method for teaching media texts. We don't so much focus on the "why" but the "what"." This was echoed by another passive faculty. "Most of the students aren't able to produce things, so we focus on digital media and technology. Basic computer literacy is our first goal ... it's more of a skills-focused course than anything."

While these faculty expressed high interest in media literacy pedagogy, their syllabi and commentary displayed their teaching approach as viewing media literacy as a 
content area rather than a pedagogy that can be taught in any context. At no point did these faculty demonstrate a willingness to create innovative instruction that allowed for active learning to take place, rather the creation of a safe environment for students to better learn how to use technologies and recreate an expected product. One of the adjunct faculty stated that he "wish[ed] I could do more production," but that he had not inquired with a superior about doing so. "Being an adjunct instructor," one interviewee stated, "for only a year or two ... I don't have [the] time or energy to redo the entire course."

The principal environmental similarity between these two groups of faculty was the lack of policy or administrative oversight in how they teach their courses. Administrative involvement was negligible for all of these courses; both maverick faculty claimed to have created their courses of their own volition, with near-total autonomy at all stages of course design and implementation. One of these faculty, affirming their autonomy, stated, "no one knows what I'm doing, and no one really cares as long as the students appear successful." The 4 passive faculty noted little to no oversight for their media literacy courses as well, aside from the 3 adjunct faculty being instructed to teach from the lesson plans already developed for their courses. All 6 faculty confirmed that there were no known policies at their institutions guiding the teaching of media literacy, whether those be institution-wide in the case of general education courses, or departmental policies, regardless of the discipline in which media literacy was part of the curriculum.

\section{Discussion}

The goal of this study was to explore the shared patterns across higher education courses in the U.S. in the ways that media literacy is being taught, the intended learning outcomes, and the environmental factors that influence its teaching. This included an in- 
depth exploration of the various disciplines in which media literacy is taught, whether as the focal point or within the context of another discipline, the theoretical frameworks guiding its instruction, and the possible differences found between public and private institutions where it is being taught. With these factors in mind, a theoretical framework was proposed and utilized to guide the study; this participatory ecological framework of media literacy was founded on critical thinking as a method for analysis, reflection, and creative production of media.

This study has found inconsistencies in the pedagogical approaches being utilized, a misunderstanding of the critical thinking and inquiry-based learning methods required for a critical field such as media literacy, and the lack of administrative oversight or policy support as key factors contributing to the discrepancies in its instruction and learning outcomes. While faculty shared their support for the inclusion of media literacy in their programs of study, as well as the importance of institutional policies guiding its pedagogy and implementation, the participants conversely tended to be hesitant to challenge the status quo or to incorporate small, significant changes into their instruction in order to elevate media learning above a base level of instruction. If media literacy as a field cannot consolidate its pedagogical approaches or achieve policy victories at an institutional level, it is destined to continue to be an often misunderstood, fringe topic, leading to students not acquiring the critical thinking skills necessary to become thriving citizens in our media-saturated society.

The environmental factors of media literacy instruction in higher education contribute to the extremes of its pedagogical implications and the faculty members teaching these courses. At both extremes, faculty are given a high level of latitude to do what 
makes sense for them: mavericks are encouraged and allowed to use their own creativity to initiate meaningful learning, while the passives are allowed and encouraged to stick with traditional lessons that have already been developed. Both of these groups have little to no oversight; they are informed they have either complete freedom in how they approach their courses or are restricted in teaching a course that was developed by a third party. The need for policy oversight and a standardized theoretical framework is evident in order to bring these two extremes together for a cohesive understanding of the teaching and learning of media literacy. Policy support at the institutional level would indicate an endorsement from administration, establishing a level of trust in an inquiry-based pedagogy such as media literacy. Additionally, the consistent utilization of a framework such as the participatory ecological framework of media literacy would allow for all faculty to have the tools necessary to teach media literacy concepts, no matter the content area in which they are teaching or their position at their institution.

Faculty reported overwhelming support for the inclusion of media literacy in their programs of study, as well as the importance of institutional policies guiding its pedagogy and implementation within the curriculum. Further, most participants reported that inconsistencies in understanding how media literacy should be viewed as a content area, the contexts in which it can be taught, and the lack of a determined theoretical framework across the subject matter area are hindering its understanding for students, other faculty, and the public. These findings are consistent with a previous study by Mihailidis (2008), which concluded that insufficient policy support in higher education, specifically, contributed to discrepancies in media literacy adoption, instruction, and learning outcomes. 
The differences in the teaching of media literacy at public and private universities is meaningful. Courses at public institutions incorporated the pedagogical approaches of analysis, reflection, and production of media almost universally, with media literacy courses taught at private institutions lagging behind. Likewise, the ten participatory methods of learning as identified by Jenkins et al. (2009) and verified by Literat (2014) were included in more courses at public institutions than private; the participatory methods of learning were found in 73 instances in the courses from public universities, while found only 62 times in the courses from private universities. The participatory learning methods of performance, multitasking, and distributed cognition had the largest noticeable gaps when comparing the two types of institutions, being taught more often at public institutions. No significant relationships were identified between maverick and passive instructors and the types of institutions at which they teach; these numbers were equally distributed between public and private institutions.

The difficulty of having students engaged in the intellectual rigor of critical thinking and the inquiry-based learning required of media literacy, while concurrently producing media texts, was noted by the majority of faculty as the main obstacle for media literacy to overcome in order for it to be seen as a more legitimate subject area in higher education settings. Most faculty, with the exception of those labeled in this study as maverick instructors, simply erred on the side of sharing information with their students rather than challenging themselves to incorporate the additional, difficult concepts required of critical media literacy pedagogy. While there was no dichotomy present in the acknowledgment that creative production and inquiry-based instruction are required to move media literacy past the point of teaching students what to think but instead how to think, the 
majority of participants in this study did not feel they had the expertise, resources, or support to take these additional steps.

\section{Conclusion and Recommendations}

Media literacy is a challenging subject to teach. If taught at a base level, students are asked to complete simple learning tasks, which is not significantly different than other subjects. A small step to ensuring media literacy becomes the inquiry-based pedagogy it intends to be is the inclusion of the three pedagogical approaches of analysis, reflection, and creative production in all courses. One solution would be the agreement of media literacy as a field to utilize a single theoretical framework, such as the participatory ecological framework of media literacy, as a starting point for all instruction; this would ensure the inclusion of the necessary pedagogical approaches, media literacy concepts, and participatory methods of learning for all faculty teaching these courses. It would be encouraged for faculty to still make their own decisions on how to utilize the framework in their courses, but this would at a minimum guarantee that all instruction has a uniform base level of pedagogical best practices from which to start.

In addition, inequities in creative licenses as to the approaches for faculty teaching these courses need to be addressed, particularly for adjunct instructors struggling to teach the subject at the level required of a critical pedagogy. Slight modifications to media literacy courses, or the incorporation of small, significant changes to current instruction would also be substantial. For example, by having opportunities for students to create their own questions and then produce something on a small scale would help media literacy embody the inquiry-based pedagogy it strives to be recognized as. 
The inclusion of media literacy in higher education curriculum through institutional policy development is also recommended; administrative advocacy would make clear the intentions and principles guiding media literacy instruction at their institutions. Issues such as consistency in pedagogy and the scope of its instruction at individual institutions could be substantiated through this policy inclusions. Additionally, the presence of media literacy in institutional policy can assist in future accreditation efforts as media literacy continues to become a more important, mainstream content area more akin to its level of prominence in other countries. These changes cannot happen in a vacuum; the field of media literacy must also reckon with its nonconformist history and begin to encourage and accept more oversight from departmental, institutional, and national educational policies to ensure any sense of stability or success. 
MEDIA LITERACY IN HIGHER EDUCATION POLICY 227

Chapter Six: Scholarly Practitioner Reflection 
The dissertation writing process has honestly been a long and arduous process for me. I began this journey energized and working at a relatively constant pace, and then life happened. A couple of years of personal chaos grounded everything to a halt. The fact that I was able to pick this work back up after nearly a two-year hiatus is, I believe, a testament to my growth as a scholar and educational leader during my time in the Statewide Cooperative EdD Program.

The dissertation process has influenced me as a scholar in that it has inspired me to both join in and initiate my own, and often competing, research projects which have led to scholarly articles and other publications. This process has, however, especially influenced me as an educational leader in my field. I began a new position at the University of Missouri nearly simultaneous to the beginning of the dissertation writing process, during which I introduced a research and practical approach to the field of instructional media pedagogy within my department. I have since become an expert in this field, able to apply my knowledge in a practical sense in my daily work, as well as sharing my expertise with others through invited conference presentations and other publications. This has led to my recent promotion to a managerial role within the Office of eLearning at the system level at the University of Missouri, where I am now able to work with a team to continue to push a research-based approach to the everyday practical work of instructional design and development.

I would like to first touch on how writing my dissertation has influenced my scholarly work. The simple fact of undertaking such a significant research project of this scale has greatly helped my ability to undertake scholarly pursuits. This dissertation has given me the first-hand experience of conducting my own research, data collection and 
analysis; while this has been a genuinely enjoyable experience, it also allowed me to make mistakes, learn from them, and then try again. Even though the process took much longer than I initially expected or hoped for, it was also a blessing in disguise as it allowed me the time and freedom to learn from my errors as I worked through the writing and research steps.

A few of the valuable lessons I learned include the importance of extreme vetting of sources, as my topic touched on issues including current events, disinformation, propaganda, and the news and media industries. It would have been easy to include fallible sources in my research, and I had to spend a lot of time researching and writing my literature review with this in mind. I also gained knowledge regarding the design of interviews to assist in data gathering as well as in ensuring internal validity. The second stage of data collection for this dissertation, namely the interview protocol, was by far the most difficult part of the dissertation process. I learned a lot about patience and perseverance while I had a challenging time lining up my interviews. I also learned that at some point I need to let go of the idea of perfection; I never was able to conduct as many interviews as I had originally hoped for. My committee chair finally got through to me the fact that what I had gathered was more than enough data to finally complete that step and move onto data analysis. Sometimes my stubbornness knows no bounds, but this dissertation has helped me to understand that sometimes good enough can be, in fact, quite good in and of itself.

These lessons, specifically those of resilience, patience and being more open to suggestions and changes have assisted me as I transition from a student researcher to a researcher-practitioner. I see these lessons connecting closely to the ideals I held at the 
beginning of this process. Around the time I started my master's degree, Douglas Rushkoff's (2010) Program or be programmed: Ten commands for a digital age was published; this treatise has guided my professional and scholarly work ever since and has been notably influential as I work through the dissertation process. It has been a sort of guide to help me determine and understand my own action logic (Rooke \& Torbert, 2005), how to adjust that logic as I gain more knowledge, and how this can help in my ability to lead.

In the first workspace I was in when I started writing this dissertation, I had Rushkoff's (2010) ten commands printed out and tacked onto the wall atop my computer screen so they were never out of sight. The concepts of complexity (you are not always correct), choice (you have the choice of none of the above), identity (be yourself), fact (always tell the truth) and openness (share, but do not steal) have always spoken to me (Rushkoff, 2010). I always attempted to keep these principles in mind as I conducted my research and wrote this dissertation. Complexity told me that no, I do not actually know everything; the entire idea of research and scholarly work is in line with this concept. I made innumerable mistakes during this process and I am not ashamed to say so. I always strive to be myself and be truthful in everything I do. This dissertation feels like a literal extension of my body; I could not have written about anything else as this topic is so near and dear to my heart. Finally, openness grounds so much of my personality and my actions. I am very open (oftentimes too much) in what I do, and sharing my knowledge and expertise with others, whether that be in a professional, personal, or instructional environment brings me the greatest of joys. Whether intentional or not, the idea of time (don't always be on) (Rushkoff, 2010) was not, at the beginning of this process, very 
important to me because I was always on time or early with everything in my life; the amount of time it has taken me to complete this dissertation proves that my mindset has changed in that regard.

Lastly, regarding how the dissertation process has influenced my scholarly work, it has significantly helped springboard my scholarly work outside of my doctoral program. It has given me both the confidence and experience to tackle independent research projects on my own. I am currently collaborating on two other research projects with faculty at the University of Missouri for eventual publication, and I do not feel that I would be prepared to work on those projects without the experience I have gained writing my dissertation.

The final piece I would like to touch on in this reflection is how writing this dissertation has influenced me as an educational leader in my field. As stated previously, the beginning of the dissertation process coincided almost simultaneously to me starting my first position at the University of Missouri. That position, like every single professional position I've ever had in my life, was newly created and I was the first person to fill that role. I have been very lucky in this regard, giving me the opportunity to share my expertise and initiate my own leadership approach at the outset of these positions, no matter the size or scope.

While I was hired as a media producer in charge of all of the instructional media coming out of my home department, I took it upon myself to make the position much more than the agreed upon requirements. Once I had a foothold in my new position, I independently dove into all of the research, scholarly work, professional publications, and best practices I could locate during that first year to help inform how I envisioned the 
position going forward. I feel that this exemplifies how the EdD program, and the dissertation process specifically, has helped influence my day-to-day work as both a professional and research-practitioner.

My department was folded into a system-wide office last year, as we combine our efforts across all four campuses in the University of Missouri system. In my current role I oversee a team of media designers who work with instructional designers to ensure high-quality, engaging learning experiences for digital learners enrolled in our online programs. While this new position has been a whirlwind of change management processes, the initiation of a learning culture where one previously did not exist (Gill, 2010), innumerable overtime hours, and too many pots of coffee the past few months, I view it as yet another opportunity to continue the processes I have enacted in all of my previous positions. Those principles of open-mindedness, patience, and resilience appear one more time in bright, bold letters as I navigate and lead the change I have been tasked to carry out yet again. Without the EdD program, the faculty and peers who have been instrumental in my personal growth and learning, and not only starting but finally completing the dissertation process, I would not be the educational leader I am today. 


\section{References}

Altheide, D. L. (1987). Ethnographic content analysis. Qualitative Sociology, 10(1), 6577.

Andersen, N. (2013). Media literacy education in Ontario. In B. S. De Abreu \& P. Mihailidis (Eds.), Media literacy education in action: Theoretical and pedagogical perspectives (pp. 20-26). Florence, KY: Routledge.

Association for Media Literacy. (2016). What is media literacy? Retrieved March 19, 2016, from http://www.aml.ca/keyconceptsofmedialiteracy/

Aufderheide, P. (1993). Media literacy: A report of the National Leadership Conference on Media Literacy. Queenstown, MD: Aspen Institute.

Austin, E. W. (2014). A bicycle riding theory of media literacy. In A. Silverblatt (Ed.), The Praeger handbook of media literacy (Vol. 2, pp. 538-543). Santa Barbara, CA: Praeger.

Banaji, S., \& Buckingham, D. (2013). The civic web: Young people, the Internet and civic participation. Cambridge, MA: The MIT Press.

Bardach, E. (2012). A practical guide for policy analysis: The eightfold path to more effective problem solving. Los Angeles, CA: SAGE.

Barlow, J. P. (1996). A declaration of the independence of cyberspace. Electronic Frontier Foundation. Retrieved from https://www.eff.org/cyberspace-independence

Bates, A. W. (2015). Teaching in a digital age. Retrieved from http://opentextbc.ca/teachinginadigitalage/ 
Bawden, D. (2008). Origins and concepts of digital literacy. In C. Lankshear \& M. Knobel (Eds.), Digital literacies: Concepts, policies, and practices (pp. 17-32). New York, NY: Peter Lang.

Bazalgette, C. (2014). England. In A. Silverblatt (Ed.), The Praeger handbook of media literacy (Vol. 2, pp. 874-883). Santa Barbara, CA: Praeger.

Bolman, L. G., \& Deal, T. E. (2008). Reframing organizations: Artistry, choice, and leadership. San Francisco, CA: Jossey-Bass.

boyd, danah. (2014). It's complicated. New Haven, CT: Yale University Press.

Brookfield, S. D. (2012a). Critical theory and transformative learning. In E. W. Taylor \& P. Cranton (Eds.), The handbook of transformative learning. San Francisco, CA: Jossey-Bass.

Brookfield, S. D. (2012b). Teaching for critical thinking: Tools and Techniques to help students question their assumptions. San Francisco, CA: Jossey-Bass.

Browne, M. N., \& Keeley, S. M. (2001). Asking the right questions : A guide to critical thinking (6th ed.). Upper Saddle River, NJ: Prentice Hall.

Buckingham, D. (2003). Media education: Literacy, learning and contemporary culture. Cambridge, UK: Polity Press.

Buckingham, D. (2005). The media literacy of children and young people: A review of the research literature on behalf of OFCOM (Project Report). London, UK: OFCOM. Retrieved from http://stakeholders.ofcom.org.uk/binaries/research/medialiteracy/ml_children.pdf 
Buckingham, D. (2006). Is there a digital generation? In D. Buckingham \& R. Willett (Eds.), Digital generations: Children, young people, and new media (pp. 1-13). Mahwah, NJ: Lawrence Erlbaum Associates, Inc.

Buckingham, D. (2007). Beyond technology: Children's learning in the age of digital culture. Cambridge, UK: Polity Press.

Buckingham, D. (2009). The future of media literacy in the digital age: Some challenges for policy and practice. In P. Verniers (Ed.), Media literacy in Europe: Controversies, challenges and perspectives (pp. 13-24). Brussels, Belgium: EuroMeduc.

Buckingham, D. (2016, September 20). Whatever happened to media literacy? Retrieved September 21, 2016, from https://davidbuckingham.net/2016/09/20/whateverhappened-to-media-literacy/

Caffarella, R. S., \& Daffron, S. R. (2013). Planning programs for adult learners: A practical guide (3rd ed.). San Francisco, CA: Jossey-Bass.

Chen, D.-T., Wu, J., \& Wang, Y. (2011). Unpacking new media literacy. Systemics, Cybernetics, and Informatics, 9(2), 84-88.

Christ, W. G. (2004). Assessment, media literacy standards, and higher education. The American Behavioral Scientist, 48(1), 92-96. https://doi.org/10.1177/0002764204267254

Cohen, D. K., \& Moffitt, S. L. (2011). The influence of practice on policy. In D. E. Mitchell, R. L. Crowson, \& D. Shipps (Eds.), Shaping education policy: Power and process (pp. 63-80). New York, NY: Routledge. 
Common Core State Standards Initiative. (2016). Common Core Standards for English Language Arts \& Literacy in History/Social Studies, Science, and Technical Subjects (p. 66). National Governors Association. Retrieved from http://www.corestandards.org/ELA-Literacy/

Considine, D. M., \& Considine, M. M. (2014). Media literacy preparation in undergraduate teacher training: An American and Australian perspective. In B. S. De Abreu \& P. Mihailidis (Eds.), Media literacy education in action: Theoretical and pedagogical perspectives (pp. 203-212). New York, NY: Routledge.

Cope, B., \& Kalantzis, M. (2009). Multiliteracies: New literacies, new learning. Pedagogies: An International Journal, 4(3), 164-195. https://doi.org/10.1080/15544800903076044

Copps, M. J. (2005). Where is the public interest in media consolidation? In R. W. McChesney, S. Newman, \& B. Scott (Eds.), The future of media: Resistance and reform in the 21st century. New York, NY: Seven Stories Press.

Creswell, J. W. (2007). Qualitative inquiry and research design: Choosing among the five approaches. Thousand Oaks, CA: Sage.

Creswell, J. W. (2014). Research design: Qualitative, quantitative, and mixed methods approaches. Thousand Oaks, CA: Sage.

De Abreu, B. S. (2014). Grasping the complexities of U.S. educational policy and the classroom. In B. S. De Abreu \& P. Mihailidis (Eds.), Media literacy education in action: Theoretical and pedagogical perspectives (pp. 193-199). New York, NY: Routledge. 
Desimone, L. E. (2009). Complementary methods for policy research. In G. Sykes, B. L. Schneider, D. N. Plank, \& T. G. Ford (Eds.), Handbook of education policy research (pp. 512-552). New York, NY: Routledge.

Dewey, J. (1938). Experience and education. New York, NY: Macmillan.

Duncan, B. (1989). Eight key concepts of media literacy (Media literacy resource guide). Toronto, Canada: Ontario Ministry of Education.

Duncan, B. (2006). Media literacy: Essential survival skills for the new millennium. School Libraries in Canada, 25(4), 31-34.

Duncan, B., \& Pungente, J. (2014). Canada. In A. Silverblatt (Ed.), The Praeger handbook of media literacy (Vol. 2, pp. 862-873). Santa Barbara, CA: Praeger.

Duran, R. L., Yousman, B., Walsh, K. M., \& Longshore, M. A. (2008). Holistic Media Education: An Assessment of the Effectiveness of a College Course in Media Literacy. Communication Quarterly, 56(1), 49-68. https://doi.org/10.1080/01463370701839198

EPPI-Centre. (2007). EPPI-Centre methods for conducting systematic reviews (p. 25). London: Evidence for Policy and Practice Information and Coordinating Centre. Retrieved from http://eppi.ioe.ac.uk/cms/default.aspx?tabid=88

Erstad, O. (2010). Educating the digital generation. Nordic Journal of Digital Literacy, January, 56-71.

European Union. (2007). A European approach to media literacy in the digital environment (No. Communication 833). Commission of the European Communities. Retrieved from http://eur-lex.europa.eu/legalcontent/EN/TXT/?uri=CELEX:52007DC0833 
Federal Communications Commission. (2015). Strategic plan 2015-2018 (p. 16).

Washington, D.C.: Federal Communications Commission.

Freedman, D. (2008). The politics of media policy. Cambridge, UK: Polity Press.

French, J. R. P., \& Raven, B. (2005). The bases of social power. In J. M. Shafritz, J. S.

Ott, \& Y. S. Jang (Eds.), Classics of organization theory (pp. 311-320). Belmont, CA: Wadsworth.

Friere, P. (2010). Pedagogy of the oppressed. New York, NY: Continuum.

Fry, K. G. (2014). What are we really teaching? Outline for an activist media literacy education. In B. S. De Abreu \& P. Mihailidis (Eds.), Media literacy education in action: Theoretical and pedagogical perspectives (pp. 125-137). New York, NY: Routledge.

Funk, S., Kellner, D., \& Share, J. (2016). Critical media literacy as transformative pedagogy. In M. N. Yildiz \& J. Keengwe (Eds.), Handbook of Research on Media Literacy in the Digital Age (pp. 1-30). Hershey, PA: Information Science Reference.

Gainer, J. S. (2010). Critical media literacy in middle school: Exploring the politics of representation. Journal of Adolescent \& Adult Literacy, 53(5), 364-373.

Gaines, E. (2010). Media literacy and semiotics. New York, NY: Palgrave Macmillan.

Gay, L. R., Mills, G. E., \& Airasian, P. W. (2009). Educational research: Competencies for analysis and applications. Upper Saddle River, NJ: Pearson.

Gill, S. J. (2010). Developing a learning culture in nonprofit organizations. Thousand Oaks, CA: Sage.

Giroux, H. A. (2001). Public spaces, private lives. Lanham, MD: Rowman \& Littlefield. 
Goodman, S. (2003). Teaching youth media: A critical guide to literacy, video production, and social change. New York, NY: Teachers College Press.

Harden, A. (2010). Mixed-methods systematic reviews: Integrating quantitative and qualitative findings (Focus Technical Brief No. 25) (p. 8). Austin, TX: National Center for the Dissemination of Disability Research.

Harris, D. N., \& Witte, J. F. (2011). The market for schooling. In D. E. Mitchell, R. L. Crowson, \& D. Shipps (Eds.), Shaping education policy: Power and process (pp. 92-16). New York, NY: Routledge.

HeeYoung Kim, J. (2016). Pedagogical approaches to media literacy education in the U.S. In M. N. Yildiz \& J. Keengwe (Eds.), Handbook of research on media literacy in the digital age (pp. 53-74). Hershey, PA: Information Science Reference.

Heins, M., \& Cho, C. (2003). Media literacy: An alternative to censorship (p. 57). New York, NY: Free Expression Policy Project. Retrieved from http://fepproject.org/policyreports/medialiteracy2d.html

Hobbs, R. (1998). The seven great debates in the media literacy movement. Journal of Communication, 48(1), 16-32.

Hobbs, R. (2010). Digital and media literacy: A plan of action (White paper). Washington, D.C.: The Knight Commission on the Information Needs of Communities in a Democracy. Retrieved from http://www.knightcomm.org/digital-and-media-literacy-a-plan-of-action/ Hobbs, R. (Ed.). (2016). Exploring the roots of digital and media literacy through personal narrative. Philadelphia, PA: Temple University Press. 
Ito, M., Horst, H., Bittanti, M., boyd, danah, Herr-Stephenson, B., Lange, P. G., ... Robinson, L. (2008). Living and learning with new media: Summary of findings from the Digital Youth Project (The John D. and Catherine T. MacArthur Foundation Reports on Digital Media and Learning) (p. 58). Cambridge, MA: The MIT Press.

Jenkins, H. (2006). Convergence culture: Where old and new media collide. New York, NY: New York University Press.

Jenkins, H., Purushotma, R., Weigel, M., Clinton, K., \& Robison, A. J. (2009). Confronting the challenges of participatory culture: Media education for the 21st century. Cambridge, MA: MIT Press.

Jolls, T. (2011). Voices of media literacy international pioneers speak. Retrieved October 11, 2016, from http://www.medialit.org/voices-media-literacy-internationalpioneers-speak

Jolls, T. (2016). Foreword. In M. N. Yildiz \& J. Keengwe (Eds.), Handbook of research on media literacy in the digital age (pp. xviii-xix). Hershey, PA: Information Science Reference.

Jones, C., \& Shao, B. (2011). The net generation and digital natives: Implications for higher education. York, UK: Higher Education Academy.

Kanter, R. M. (1994). Collaborative advantage: The art of alliances. Harvard Business Review, 72(4), 96-108.

Kanter, R. M. (2005). Power failure in management circuits. In J. M. Shafritz, J. S. Ott, \& Y. S. Jang (Eds.), Classics of organization theory (6th ed., pp. 342-351). Belmont, CA: Wadsworth. 
Kellner, D., \& Share, J. (2005). Toward critical media literacy: Core concepts, debates, organizations, and policy. Discourse: Studies in the Cultural Politics of Education, 26(3), 369-386.

Kleiner, B., Thomas, N., \& Lewis, L. (2007). Educational technology in teacher education programs for initial licensure (No. NCES 2008040) (p. 41). U.S. Department of Education. Retrieved from http://nces.ed.gov/pubsearch/pubsinfo.asp?pubid=2008040

Koltay, T. (2011). The media and the literacies: Media literacy, information literacy, digital literacy. Media, Culture \& Society, 33(2), 211-221. https://doi.org/10.1177/0163443710393382

Krippendorff, K. (2004). Content analysis: An introduction to its methodology (2nd ed.). Thousand Oaks, CA: SAGE.

Kubey, R., \& Baker, F. (1999). Has media literacy found a curriculuar foothold? Education Week, 19, 56-58.

Lankshear, C., \& Knobel, M. (2008). Digital literacies: Concepts, policies, and practices. New York, NY: Peter Lang.

Literat, I. (2014). Measuring new media literacies: Towards the development of a comprehensive assessment tool. Journal of Media Literacy Education, 6(1), 2.

Livingstone, S. (2002). Young people and new media: Childhood and the changing media environment. London, UK: SAGE.

Livingstone, S. (2004). Media literacy and the challenge of new information and communication technologies. The Communication Review, 7(1), 3-14. 
Livingstone, S., \& Thumim, N. (2003). Assessing the media literacy of UK adults: A review of the academic literature (p. 47). Retrieved from http://eprints.lse.ac.uk/21673/1/Assessing_the_media_literacy_of_UK_adults.pdf

Livingstone, S., \& Wang, Y.-H. (2014). On the difficulties of promoting media literacy. In B. S. De Abreu \& P. Mihailidis (Eds.), Media literacy education in action: Theoretical and pedagogical perspectives (pp. 161-172). New York, NY: Routledge.

Lundgren, P. (2013). Towards a European network for media literacy: A Nordic perspective on challenges in a global society. In B. S. De Abreu \& P. Mihailidis (Eds.), Media literacy education in action: Theoretical and pedagogical perspectives (pp. 61-68). Florence, KY: Routledge.

Lunt, P., \& Livingstone, S. (2012). Media regulation. London, UK: SAGE.

Malen, B. (2011a). An enduring issue: The relationship between political democracy and educational effectiveness. In D. E. Mitchell, R. L. Crowson, \& D. Shipps (Eds.), Shaping education policy: Power and process (pp. 23-60). New York, NY: Routledge.

Malen, B. (2011b). An enduring issue: The relationship between political democracy and educational effectiveness. In D. E. Mitchell, R. L. Crowson, \& D. Shipps (Eds.), Shaping education policy: Power and process (pp. 23-60). New York, NY: Routledge.

Martin, A. (2006). Literacies for the digital age. In A. Martin \& D. Madigan (Eds.), Digital Literacies for Learning (pp. 3-25). London, UK: Faceet. Masterman, L. (1985). Teaching the media. London, UK: Routledge. 
Masterman, L. (1997). A rationale for media literacy. In R. Kubey (Ed.), Media literacy in the information age: Current perspectives (pp. 15-68). New Brunswick, NJ: Transaction.

McChesney, R. W. (2003). Theses on media deregulation. Media, Culture \& Society, 25(1), 125-133. https://doi.org/10.1177/0163443703025001595

McLuhan, M. (1964). Understanding media: The extensions of man. New York, NY: McGraw-Hill.

Merriam, S. B. (2009). Qualitative research: A guide to design and implementation. San Francisco: Jossey-Bass.

Mihailidis, P. (2005). Media literacy in journalism/mass communication education: Can the U.S. learn from Sweden? Journalism \& Mass Communication Educator, 60(4), 415-428. https://doi.org/10.1177/107769580506000409

Mihailidis, P. (2008). Are we speaking the same language? Assessing the state of media literacy in U.S. higher education. Studies in Media \& Information Literacy Education, 8(4), 1-14. https://doi.org/10.3138/sim.8.4.001

Mihailidis, P. (2009). Beyond cynicism: Media education and civic learning outcomes in the university. International Journal of Learning and Media, 1(3), 1-14. https://doi.org/10.3138/sim.8.4.001

Mihailidis, P. (2014a). A case for curation as a media literacy imperative for participatory culture. In B. S. De Abreu \& P. Mihailidis (Eds.), Media literacy education in action: Theoretical and pedagogical perspectives (pp. 29-36). New York, NY: Routledge. 
Mihailidis, P. (2014b). Media literacy and the emerging citizen. New York, NY: Peter Lang.

Morrell, E., Dueñas, R., Garcia, V., \& Lopez, J. (2013). Critical media pedagogy:

Teaching for achievement in city schools. New York, NY: Teacher's College Press.

National Association for Media Literacy Education. (n.d.). Aims \& Scope. Retrieved October 17, 2016, from http://digitalcommons.uri.edu/jmle/aimsandscope.html National Association for Media Literacy Education. (n.d.). Media Literacy Defined. Retrieved May 21, 2016, from https://namle.net/publications/media-literacydefinitions/

National Association for Media Literacy Education. (n.d.). MLE \& Common Core Standards. Retrieved June 6, 2016, from https://namle.net/publications/mlecommon-core-standards/

National Association for Media Literacy Education. (2007). Core principles of media literacy education in the U.S. National Association for Media Literacy Education. Retrieved from http://namle.net/publications/core-principles/

National Association for Media Literacy Education. (2014). Media literacy education \& the common core state standards. National Association for Media Literacy Education. Retrieved from http://namle.net/publications/mle-common-corestandards/

Nkana, S. (2014). History of U.S. media literacy education. In A. Silverblatt (Ed.), The Praeger handbook of media literacy (Vol. 2, pp. 544-550). Santa Barbara, CA: Praeger. 
Northouse, P. G. (2013). Leadership: Theory and practice. Los Angeles, CA: SAGE.

Office of Educational Technology. (2016). Future ready learning: Reimagining the role of technology in education (National Educational Technology Plan) (p. 106). U.S. Department of Education. Retrieved from http://tech.ed.gov/

Papacharissi, Z. A. (2010). A private sphere: Democracy in a digital age. Cambridge, UK: Polity Press.

Papathanassopoulos, S., \& Negrine, R. (2011). European media: Structures, policies and identity. Cambridge, UK: Polity Press.

Parliament of the United Kingdom. Communications Act 2003, Chapter 212003 c 21 (2003).

Pensky, M. (2001). Digital natives, digital immigrants. On the Horizon, 9(5), 1-6.

Phang, A., \& Schaefer, D. J. (2009). Is ignorance bliss? Assessing Singaporean media literacy awareness in the era of globalization. Journalism \& Mass Communication Educator, 64(2), 156-172. https://doi.org/10.1177/107769580906400203

Potter, W. J. (2004). Theory of media literacy: A cognitive approach. Thousand Oaks, CA: SAGE.

Potter, W. J. (2013). Media literacy (6th ed.). Thousand Oaks, CA: SAGE.

Powers, E., Moeller, S., \& Yuan, Y. (2016). Political engagement during a presidential election year: A case study of media literacy students. Journal of Media Literacy Education, 8(1), 1-14.

RobbGrieco, M. (2014). Why history matters for media literacy education. Journal of Media Literacy Education, 6(2), 3-22. 
RobbGrieco, M., \& Hobbs, R. (2013). A field guide to media literacy education in the U.S. (White paper). Media Education Lab.

Rosenbaum, J. E., \& Beentjes, J. W. J. (2004). Reconceptualizing media literacy. In K. Renckstorf, D. McQuail, J. E. Rosenbaum, \& G. Schaap (Eds.), Action theory and communication research: Recent developments in Europe (pp. 141-162). Berlin: Mouton de Gruyter.

Rosenfeld, K. N. (2016). Terms of the digital age: Realities and cultural paradigms. In M. N. Yildiz \& J. Keengwe (Eds.), Handbook of research on media literacy in the digital age (pp. 115-144). Hershey, PA: Information Science Reference.

Rooke, D., \& Torbert, W. R. (2005). Seven transformations of leadership. In HBR's 10 must reads on leadership (pp. 137-161). Harvard Business Review Press.

Rushkoff, D. (2010). Program or be programmed: Ten commands for a digital age. New York, NY: OR Books.

Sandelowski, M., Voils, C. I., \& Barroso, J. (2006). Defining and designing mixed research synthesis studies. Research in the Schools, 13(1), 15.

Sarikakis, K. (2007). The place of media and cultural policy in the EU (European Studies No. 23) (pp. 13-22).

Scharrer, E. (2002). Making a case for media literacy in the curriculum: Outcomes and assessment. Journal of Adolescent \& Adult Literacy, 46(4), 354-358.

Scheibe, C., \& Rogow, F. (2012). The teacher's guide to media literacy: Critical thinking in a multimedia world. Thousand Oaks, CA: Corwin.

Schilder, E., Lockee, B., \& Saxon, D. P. (2016). The challenges of assessing media literacy education. Journal of Media Literacy Education, 8(1), 32-48. 
Schmidt, H. C. (2012). Essential but problematic: Faculty perceptions of media literacy education at the university level. Qualitative Research Reports in COmmunication, 13(1), 10-20. https://doi.org/10.1080/17459435.2012.719204

Schmidt, H. C. (2013). Media literacy education from kindergarten to college: A comparison of how media literacy is addressed across the educational system. Journal of Media Literacy Education, 5(1), 295-309.

Schwarz, G. (2014). Rhetoric in a new key: Media literacy education for the twenty-first century university. In B. S. De Abreu \& P. Mihailidis (Eds.), Media literacy education in action: Theoretical and pedagogical perspectives (pp. 213-218). New York, NY: Routledge.

Seiter, E. (2007). The internet playground: Children's access, entertainment, and miseducation. New York, NY: Peter Lang.

Senge, P. M. (2005). The fifth discipline: A shift of mind. In J. M. Shafritz, J. S. Ott, \& Y. S. Jang (Eds.), Classics of organization theory (6th ed., pp. 441-459). Belmont, CA: Wadsworth.

Silverblatt, A. (Ed.). (2014). The Praeger handbook of media literacy (Vol. 1). Santa Barbara, CA: ABC-CLIO, LLC.

Silverblatt, A., Ferry, J., \& Finan, B. (2009). Approaches to media literacy: A handbook (2nd ed.). Armonk, NY: M.E. Sharpe.

St. John, E. P., Daun-Barnett, N., \& Moronski-Chapman, K. M. (2013). Public policy and higher education: Reframing strategies for preparation, access, and college success. New York, NY: Routledge. 
Stuhlman, L., \& Silverblatt, A. (2007). Media literacy in U.S. institutions of higher education: Survey to explore the depth and breadth of media literacy education. Retrieved from http://www.webster. edu/medialiteracy/Media\%20Literacy\%20 Presentation2.ppt

Swanson, C. B. (2009). An applied perspective on research, method, and policy. In G. Sykes, B. Schneider, \& D. N. Plank (Eds.), Handbook of Education Policy Research (pp. 212-219). New York: Routledge.

Swedish Media Council. (2016). Statens medieråd. Retrieved March 19, 2016, from http://statensmedierad.se/

The College Board. (n.d.). The College Board English Language Arts Framework (p. 77). New York, NY: The College Board. Retrieved from https://www.collegeboard.org/sites/default/files/english-language-arts-frameworkacademic-advisory-committee.pdf

The London School of Economics and Political Science. (2016). Professor Sonia Livingstone. Retrieved October 12, 2016, from http://www.lse.ac.uk/media@1se/WhosWho/AcademicStaff/soniaLivingstone.asp $\mathrm{X}$

Thoman, E., \& Jolls. (2003). Literacy for the 21st century: An overview and orientation guide to media literacy education (p. 50). Malibu, CA: Center for Media Literacy.

Tufte, B. (1998). Tv pa tavlm: Orrr bem, skole og mcdier. Copenhagen, Denmark: Akademisk forlag.

Van Heertum, R., \& Share, J. (2006). A new direction for multiple literacy education. McGill Journal of Education, 41(3), 249-265. 
VideoBlocks EDU. (2015). The 2016 state of digital media in higher education (White paper) (p. 23). VideoBlocks EDU.

Wallis, R., \& Buckingham, D. (2013). Arming the citizen-consumer: The invention of “media literacy”within UK communications policy. European Journal of Communication, 28(5), 527-540.

Weimer, D. L. (2009). Making education research more policy-analytic. In G. Sykes, B. Schneider, \& D. N. Plank (Eds.), Handbook of Education Policy Research (pp. 93-100). New York: Routledge.

Yates, B. L. (2004). Media literacy and the policymaking process: A framework for understanding influences on potential educational policy outputs. Studies in Media \& Information Literacy Education, 4(2), 1-14. 
Brad Mitchell earned his B.S. in Mass Media Studies (2003) and M.Ed. in Educational Technology (2013) from Missouri State University, as well as a specialized certificate in Sound Design for Visual Media from the Vancouver Film School (2004). He is currently an Ed.D. doctoral candidate in Educational Leadership and Policy Analysis at the University of Missouri with an academic focus on media literacy pedagogy, assessment and policy; and instructional media pedagogy and technologies.

He began his career working in the media industry in the New York City metropolitan area and in Switzerland from 2005 to 2008 . When he moved back to the U.S., he transitioned to the field of higher education as the New Media Producer at Missouri State University from 2008 to 2014. In 2015, he began working as the Media Production Coordinator in Educational Technologies at the University of Missouri. In 2019, Educational Technologies was folded into a new system-wide office, now known as the Office of eLearning. Brad was named the new Manager of Media Design on the Program and Course Development Services team in August 2020.

Brad has been an occasional adjunct professor since 2012, teaching courses at Missouri State University and the University of Missouri in colleges of education, firstyear foundations, and most recently in the Missouri School of Journalism, teaching Applied Projects for Journalism and Strategic Communication. His service at the University of Missouri includes membership on the Excellence in Teaching with Technology Awards committee, as well as facilitating sessions in the Online Teaching Foundations 
program, focusing on helping faculty become better able to create effective instructional media on their own.

In 2016, he was part of the team that received the College-Level Course Award from the Association for Distance Education and Independent Learning (ADEIL). In 2019, he received the Excellence in Support Service Award from ADEIL, and in 2020 he was honored with the Excellence in Instructional Design Award from the University Professional \& Continuing Education Association (UPCEA). He has been recognized for sharing his expertise at national education conferences as an invited speaker as well as conducting hands-on media and video production workshops. He is known for bringing a research-based approach to the production of instructional media with a focus on pedagogy and student outcomes. 